\title{
PRODUCTION OF NATURAL GAS AND FLUID FLOW IN TIGHT SAND RESERVOIRS
}

\author{
FINAL TECHNICAL REPORT
}

REPORTING PERIOD: JUNE 30, 2004 TO JUNE 30, 2006

AUTHOR: MARIA CECILIA BRAVO

ISSUED: JULY 2006

DE-FG26-04NT42132 


\section{DISCLAIMER}

"This report was prepared as an account of work sponsored by an agency of the United States Government. Neither the United States Government nor any agency thereof, nor any of their employees, makes any warranty, express or implied, or assumes any legal liability or responsibility for the accuracy, completeness, or usefulness of any information, apparatus, product, or process disclosed, or represents that its use would not infringe privately owned rights. Reference herein to any specific commercial product, process, or service by trade name, trademark, manufacturer, or otherwise does not necessarily constitute or imply its endorsement recommendation, or favoring by the United States Government or any agency thereof. The views and opinions of authors expressed herein do not necessarily state or reflect those of the United States Government or any agency thereof.” 


\section{ABSTRACT}

This document reports progress of this research effort in identifying relationships and defining dependencies between macroscopic reservoir parameters strongly affected by microscopic flow dynamics and production well performance in tight gas sand reservoirs. These dependencies are investigated by identifying the main transport mechanisms at the pore scale that should affect fluids flow at the reservoir scale. A critical review of commercial reservoir simulators, used to predict tight sand gas reservoir, revealed that many are poor when used to model fluid flow through tight reservoirs. Conventional simulators ignore altogether or model incorrectly certain phenomena such as, Knudsen diffusion, electro-kinetic effects, ordinary diffusion mechanisms and water vaporization. We studied the effect of Knudsen's number in Klinkenberg's equation and evaluated the effect of different flow regimes on Klinkenberg's parameter $b$. We developed a model capable of explaining the pressure dependence of this parameter that has been experimentally observed, but not explained in the conventional formalisms. We demonstrated the relevance of this, so far ignored effect, in tight sands reservoir modeling. A 2-D numerical simulator based on equations that capture the above mentioned phenomena was developed. Dynamic implications of new equations are comprehensively discussed in our work and their relative contribution to the flow rate is evaluated. We performed several simulation sensitivity studies that evidenced that, in general terms, our formalism should be implemented in order to get more reliable tight sands gas reservoirs' predictions. 


\section{TABLE OF CONTENTS}

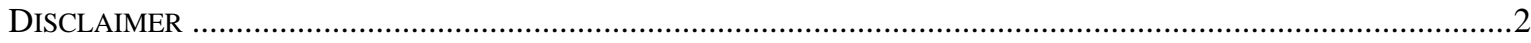

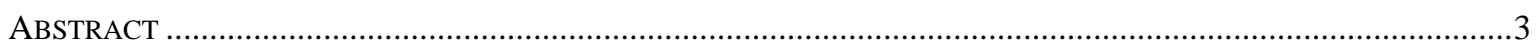

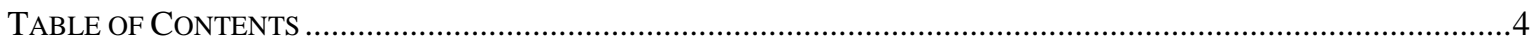

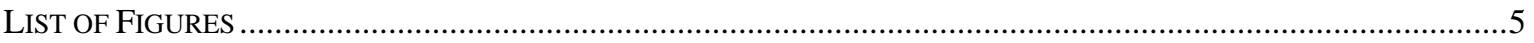

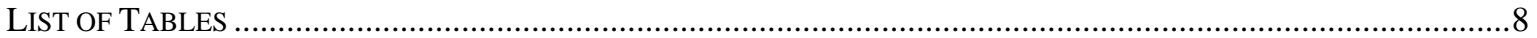

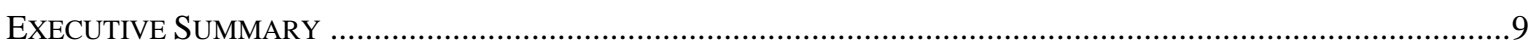

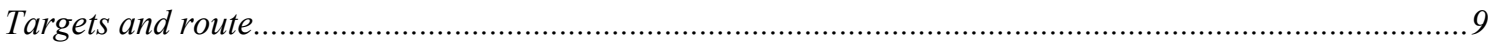

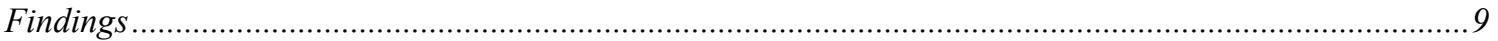

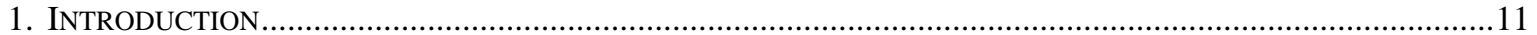

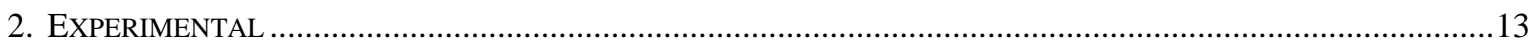

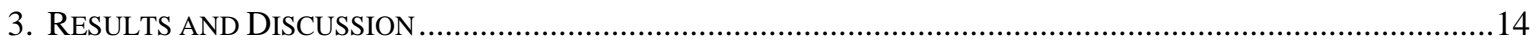

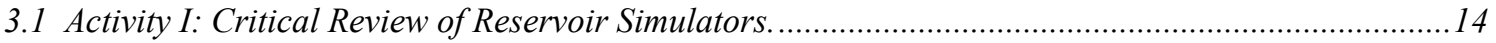

3.1.1 Multi-Mechanistic Approach.................................................................................................................. 18

3.1.2 Magnitude Orders’ Analysis................................................................................................................... 32

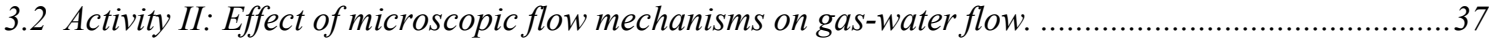

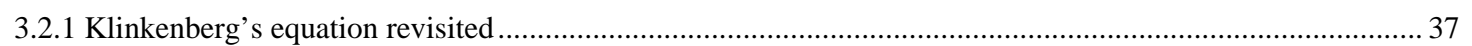

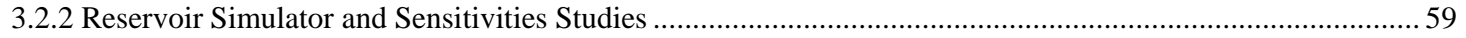

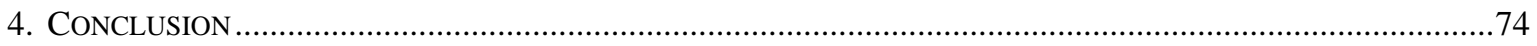

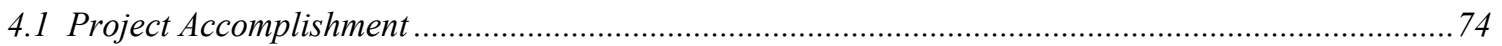

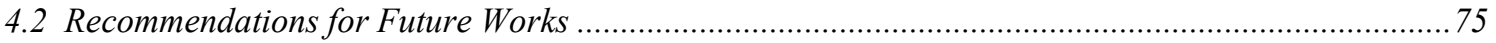

NOMENCLATURE, ACRONYMS AND ABBREVIATIONS .....................................................................................

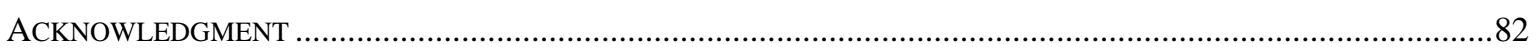

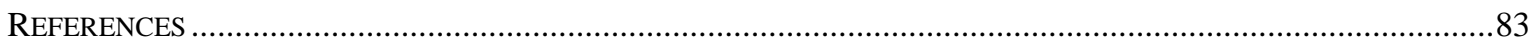




\section{LIST OF FIGURES}

Figure 1. Three main types of pore geometry of tight sand-stones. (a) Grain supported pores, (b) Slot and solution pores, (c) Matrix supported grains. Taken from Ref. 15.

Figure 2. Microscopic sections of (a) Conventional, and (b) Tight sandstone. Conventional sandstone has well- connected pores (dark blue). Tight gas sandstone pores are irregularly distributed and weakly connected. Taken from www.netl.doe.gov.

Figure 3. Representations of the phase volumes at reservoir conditions and at standard conditions after a flash depressurization of each phase separately. 20

Figure 4. Plot of the Klinkenberg permeability versus the Knudsen diffusion coefficient for air (nitrogen) flow. Points are experimental results and the lines are available correlations, including the solid line corresponding to equation (38) 25

Figure 5. Experimental data for longitudinal dispersion coefficient $D_{L}$ vs Peclet numbers Pe ( $=d_{g} v / \mathrm{D}_{\mathrm{m}}$, with $d_{g}$ as the average diameter of a grain, $v$, velocity and $\mathrm{D}_{\mathrm{m}}$, molecular diffusivity) in five different regimes. $\mathrm{D}_{\mathrm{m}}$ is molecular diffusivity. Taken from Ref. $51 \ldots . . .28$

Figure 6. Maximum amount of salt that can be dissolved in liquid (brine) according to Ref. 63 ........ 31

Figure 7. Iso-lines of $\left(V_{D_{k g}}+V_{D_{k l}}\right) /\left(V_{C_{k g}}+V_{C_{k l}}\right)$. (a) Methane $(k=m)$ from Equation (65) and (b) Water $(k=w)$ from Equation (66). Continuous line for $70{ }^{\circ} \mathrm{F}$, dot-dashed line for $240{ }^{\circ} \mathrm{F}$ and dotted line for $400{ }^{\circ} \mathrm{F}$. 35

Figure 8. Iso-lines of the ratio $V_{K n_{m l}} / V_{P C_{m l}}=b / P_{g}$ (from equation 9) in a $\left(P, K_{\infty}\right)$ space. 36

Figure 9. Iso-lines of the ratio (a) $\left(V_{D_{w g}}+V_{C_{w g}}\right) /\left(V_{D_{w l}}+V_{C_{w l}}\right)$ and (b) $V_{D_{w g}} / V_{C_{w g}}$. Continuous line for $70{ }^{\circ} \mathrm{F}$, dot-dashed line for $240{ }^{\circ} \mathrm{F}$ and dotted line for $400{ }^{\circ} \mathrm{F}$. 37

Figure 10. Representation of velocity profiles in a capillary. 44

Figure 11. Klinkenberg parameter $b$ vs. mean pressure $P_{g}$. Experimental data and theoretical predictions based on different velocity profiles in the non-Poisseuille flow regime region.49

Figure 12. Velocity profiles inside a capillary for different Knudsen Numbers vs velocity ratio, in case of considering linear profile in the non-Poisseuille region.

Figure 13. Dimensionless parameter $b^{*}$ considering linear velocity profile in the non-Poisseuille region. Numbers at right represent the values of $n$. Notice that $p^{*}=1 / w \mathrm{Kn}$. 52 
Figure 14. Theoretical evaluation of scenarios c) $p_{0}^{*}=1$ and d) $p_{0}^{*}=5$ in Figure 13. The ordinate, which was obtained from equation (125), is $w \mathrm{Kn}$ for c) and $w \mathrm{Kn} / 5$ for $\mathrm{d}$ ).

Figure 15. Klinkenberg parameter $b$, obtained from experimental data ${ }^{13}$ considering three different values of Klinkenberg permeability, $K_{\infty}$. Black for $K_{\infty}=0.024 m D$, red for $K_{\infty}=0.026 m D$ and blue for $K_{\infty}=0.030 m D$. 54

Figure 16. Klinkenberg parameter $b$ vs pressure. (a) $K_{\infty}=2.36 m D$, (b) $K_{\infty}=23 m D$. Dots for experimental data ${ }^{12}$ and lines for theoretical curves from equation (101) for $f_{0}=1$ and parameters shown in table 3. .55

Figure 17. Error of $b^{*}$ (from equation (112)) vs the dimensionless parameter $p_{0}^{*}$ for $n=1.5$ (double dotted dashed line), $n=2$ (solid line), $n=3$ (dotted-dashed line), $n=5$ (dotted line) and $n=10$ (dashed line). Between both gray vertical lines is the region of expected $p_{0}^{*}$ values for tight sand. 58

Figure 18. Error in the flow rate of gas (Eq. (55)) vs $p^{*}$. Values $\left(p_{0}^{*}, n\right)$ that originated the curves are shown on them.

Figure 19. Effect of consider air or methane under different temperatures. (a) Gas viscosity, (b) Klinkenberg parameter $b$ with $D^{K} / K_{\infty}=4.41 \times 10^{10} s^{-1}$ for black and $D^{K} / K_{\infty}=2.84 \times 10^{10} s^{-1}$ for the others curves.

Figure 20. Klinkenberg parameter $b$ (from equation (101)) vs pressure for all permeabilities tested in the simulations.

Figure 21. Ratio of Gas permeability to Klinkenberg permeability vs. distance to the well relative to the well radius. Continuous line for matrix $\left(K_{g}^{M} / K_{\infty}^{M}\right)$ and dashed line for fracture $\left(K_{g}^{F} / K_{\infty}^{F}\right)$

Figure 22. Cumulated gas production (MMSCF) vs time for three different $b: b_{1}=0, b_{2}$ given by equations (37), and $b_{3}$ dependent on pressure, by equation (101). (a) for base case, $K_{\infty}^{F}=K_{\infty}^{M}$, (b) plot (a) in a different scale, (c) $K_{\infty}^{F}=1000 K_{\infty}^{M}$ and (d) plot (a) in a different scale. .71 
Figure 23. Cumulated gas production (MMSCF) vs time. $b_{1}=0, b_{2}$ given by equations (37), and $b_{3}$ dependent on pressure, by equation (101). (a) for $K_{\infty}^{M}=0.0001 m D, K_{\infty}^{F}=K_{\infty}^{M}$, (b) plot (a) in a different scale, (c) $K_{\infty}^{F}=1000 K_{\infty}^{M}$ and (d) plot (a) in a different scale................. 72

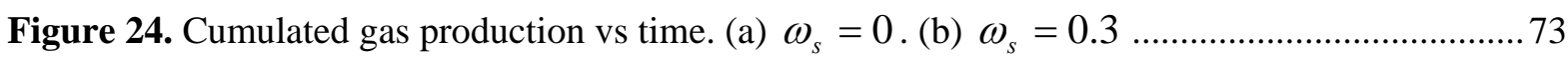




\section{LIST OF TABLES}

Table 1. Each component's characteristic parameters. Blank boxes correspond to unused parameters.

Table 2. Parameters involved on the theoretical ranges of the dimensionless quantities..................... 51

Table 3. Parameters determined for matching our theoretical approach and experimental data. ${ }^{\mathbf{1 2} 13} \ldots 55$

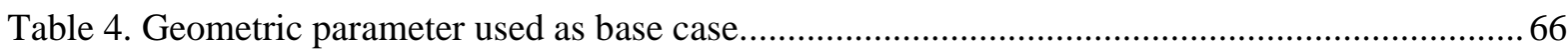

Table 5. Reservoir and flow parameters used as base case. ${ }^{\mathrm{a}}$ Total porosity equal to $0.069 .{ }^{\mathrm{b}}$ considered

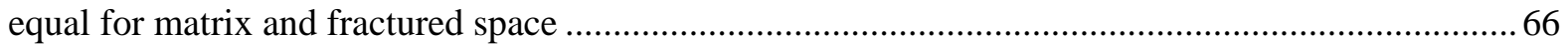

Table 6. Initial and boundary conditions for base case. ${ }^{a}$ No flow through the boundary allowed. ...... 66

Table 7. Data related to well production history used for the base case ….........................................67 67 


\section{EXECUTIVE SUMMARY}

\section{TARGETS AND ROUTE}

The objective of this research effort was to find relationships among macroscopic reservoir parameters, production well performance and the microscopic phenomena, in tight gas sand reservoirs. To achieve this goal, Applied Research Center (ARC) at Florida International University completed the following activities.

$>$ Critical review of reservoir simulators used to predict tight sands reservoirs performance. More than 270 revised publications (papers, theses and reports) gave us a good picture of the state-of-the-art in this matter. Range of applicability of multiphase flow equations of the different approaches were studied as well as the impact of some microscopic flow mechanism on gas-water transport in these low-permeability rocks.

$>$ Evaluation of pore size effect on the relative gas-water flow efficiency. Development and implementation of simulation and modeling techniques able of explaining unexpected behaviors of tight sand gas reservoirs. Phenomena such as Knudsen diffusion, electrokinetic effects, ordinary diffusion mechanisms and water vaporization were included in this study. Subsequently, ARC evaluated the impact of fluid and reservoir properties on fluid flow dynamic behavior.

\section{FINDINGS}

We develop a formulation that includes:

$>$ Diffusive (Knudsen and ordinary) mechanism of flow.

$>$ Different flow regimes at pore scale (from Knudsen to free molecular flow).

$>$ Water phase transitions (water vaporization).

$>$ Salt content variation due to water vaporization.

$>$ Dual-porosity and dual-permeability porous medium.

Reservoir simulators that ignore these aspects are, in general terms, unreliable for tight sand gas reservoir performance prediction. The relevance of these aspects on the lack of prediction of conventional reservoir simulators is strongly dependent on pressure, temperature, permeability and fractures density. Reservoir's pressure varies with distance to well and production time, thus, during the life-time of a reservoir, we will have a dynamic relative relevance of the phenomena described above. Maps of relative contribution of diffusive and convective mechanisms for a practical range of Klinkenberg permeabilities and pressures were obtained. These maps allow preliminary assessing the reliability that conventional simulators would offer in each particular case. For instance, unexpected water production from Bossier sands (Mimms Creek and Dew Fields) was suggested (based on practical evidences) to be due to condensed water vapor. Our maps confirm that for the type of rocks, pressures and temperature of these fields, the flow of water vapor goes from influencing to dominating the water production. In this scenario, the diffusive mechanism of flow goes from irrelevant to affecting the water flow dynamic. Moreover, the unexpected gas production rates could have been predicted by a simulator based on the multi-mechanistic formulation here offered, because, as inferred from the maps, the diffusive mechanisms (ordinary and Knudsen) of flow go from irrelevant to impacting the gas flow dynamics and consequently the gas production. Thus, in general terms, simulators based on formalisms that ignore the above detailed aspects are unreliable.

The slippage effect and, more specifically, the way how it is considered in the macroscopic equations, was revisited in order to explain some inconsistencies observed in the literature reviewed. 
We noticed that the so far ignored pressure dependence, experimentally observed of the parameter b, could be of considerable relevance for the dynamic behavior of gases in the reservoirs. We suggest a practical and physically-supported model that allows considering the pressure effect on the parameter $\boldsymbol{b}$. Our model was validated with experimental data obtained for tight sands and other conventional porous media. An evaluation of the effect of this pressure dependence in the gas flow rate, showed that, for Knudsen numbers (mean free path divided by capillary radius) in the range $10^{-2}<\mathrm{Kn}<10^{2}$, the pressure dependence of $\boldsymbol{b}$ can not be ignored, or, errors on flow rate predicted could be up to larger than a $100 \%$. Out of this range of Knudsen numbers the assumption that $b$ does not depend on pressure, is a good approximation.

We developed a 2-D numerical simulator, based on the proposed formalism, to evaluate on dynamic bases, the effect of the above mentioned considerations on the predictions of fluid flow. Simulations of a hypothetic reservoir, with properties similar to those reported for Upper Cozzette Blanket sands, were performed. Results indicate that if the pressure dependence of the Klinkenberg parameter $\boldsymbol{b}$ is ignored, cumulated gas produced is under-predicted around $25 \%$ for matrix permeabilities of $0.001 \mathrm{mD}$. However, if this permeability only corresponds to the matrix permeability and the fracture permeability is considered 1000 times of it, instead of under-prediction we observe around $10 \%$ over-prediction, if the mentioned pressure dependence is ignored. Similar studies but for a permeability of $0.0001 \mathrm{mD}$, show that the under-prediction of cumulated gas production is around $90 \%$, while for fracture permeability of 10 times the matrix permeability the under-estimation is around $30 \%$. In this case if fracture permeability is 1000 times the matrix permeability an over estimation near of $10 \%$ is also observed. Our estimations do not consider the effect of water saturation (around $40 \%$ in the case here studied) effect on the Klinkenberg permeability, because, in our opinion, further studies should be made on the fluids distribution at pore scale to determine if the reduction in permeability can be associated to a pore size reduction.

Performed sensitivities to the phase transitions of salts show that salts depositions, which reduce the permeability of matrix and fracture systems, were observed from a salt weight fraction near to 0.1. A drastic reduction in cumulated gas production, from about 80 MMSCF to about 4 MMSCF was obtained for a salt weight fraction of 0.3 .

It is worth noting that the proposed formulation may have impacts beyond that of natural gas flow in tight sands. Coal-bed methane and shale gas reservoirs are also low-permeabilities systems, where the phenomena here studied are playing important roles. Other areas that could benefit from this more realistic model of the gas transport through tight porous systems are: heterogeneous catalysis and adsorption problems in situ remediation techniques for removal of Volatile Organic Compound (VOCs) and Non-Aqueous Phase Liquid (NAPLs), prediction of gas transport into surrounding media from the disposal of hazardous waste sites, among others. 


\section{INTRODUCTION}

The forecast of U.S. natural gas demand indicate an increase of around 17 percent by $2025 .{ }^{1}$ Most of the additional supply needed for domestic natural gas production will come from low-permeability reservoirs, such as tight sands, coal-bed methane and shale gas reservoirs. The understanding of the dynamic of gases in these low-permeability systems needs to be improved to enable a more accurate appraisal, forecast of production, and further development of these potential resources.

Production forecasts have traditionaly been based on reservoir simulations studies. The main goal of a reservoir simulator is to predict future performance of the reservoir and to help in the study of alternatives for increasing the final recovery. ${ }^{2}$ However, commercial reservoir simulators could be inappropriate to predict low-permeability reservoir behaviors, because the transport equations on which they are based, do not consider properly some rock-fluids interactions, which may be dominating the flow dynamics in these reservoirs. Previous works on this topic indicate that more reliable and physically supported modeling is required. ${ }^{3.6}$

The study of the impact of microscopic flow mechanisms on gas production parameters in tight sand reservoirs can help identify possible relationships and dependencies between macroscopic reservoir parameters and the well's performance. Subsequently, the study can be used in the development of rigorous, macroscopic equations that more accurately describe the fluid's flow behavior in tight gas reservoirs and allow operators to better assess exploitation strategies, predict well performance, and avoid unexpected fluids production, such as associated water production or varying gas production.

The purpose of this project is to analyze, evaluate, and improve current modeling approaches for simulation of tight sands gas reservoirs, which have in-situ permeability less than $1 \mathrm{mD} .^{7}$ Special emphasis is given to certain microscopic phenomena that become relevant in this type of reservoirs under certain conditions. We performed a critical review, which included engineering areas that have been very active in the modeling and simulation of fluids in porous media, such as: heterogeneous catalysis and adsorption problems, ${ }^{8}$ in situ remediation techniques for removal of Volatile Organic Compound (VOCs) and Non-Aqueous Phase Liquid (NAPLs), ${ }^{9,10}$ and prediction of gas transport into surrounding media from the disposal of hazardous waste sites. ${ }^{11}$

The critical review was focused on evaluating the predictive capability of commercial reservoir simulations when used in studies of tight sand gas reservoir. Section 3.1.2 summarizes and discusses the most relevant information collected, while section 3.1.2 offers analysis of orders of magnitudes that estimates the relative weighs of the different mechanisms of flow considered in the multimechanistic formulation proposed. 
We found misinterpretation in the commonly termed Klinkenberg effect, which could lead to large errors of gases flow predictions in tight sand reservoirs. Section 3.2.1 of this report contains a detailed description of the conventional approaches to estimate the Klinkenberg parameter $b$, and our approach. A model that explains, the so far ignored, pressure dependence of this parameter is offered and discussed. Reported experiments in the scientific literature were used to validate our formulation. Finally, we developed a 2-D numerical simulation to quantify, on dynamic bases, the importance of phenomena at pore-scale, on the predictions of fluid flow at reservoir scale. Section 3.2.2, presents the details of the reservoir simulator developed, as well as sensitivities studies performed. 


\section{EXPERIMENTAL}

The scope of work covered by this grant does not contemplate any experimental laboratory work. All the work performed is theoretical and based on analytical models of the fluid flow phenomenon. However, we do use readily available experimental data to validate the models we developed. ${ }^{12,13}$ 


\section{RESULTS AND DISCUSSION}

\subsection{ACTIVITY I: CRITICAL REVIEW OF RESERVOIR SIMULATORS.}

The critical review of reservoirs simulators utilized to predict gas tight-sands fields' production was divided in three major areas:

1. Reservoirs characterization: Permebilities, porosities, capillary pressure and relative permeability, pores sizes and morphology, spatial distribution, clays content, water properties, pore volume compressibility, gas slippage effect, sensitivity of permeability to overburden pressure, etc

2. Relevant field experiences: Water production, long-flowing wells decline curves analysis, role of natural and artificial fractures, infill well, etc.

3. Reservoir modeling and simulation: Multiphase transport equations, closure relations, physicals and numerical assumptions, etc.

A careful analysis of these aspects confirmed that discrepancies between the assumptions behind conventional reservoir simulators utilized to predict tight sands reservoir's performance and fluidrock characteristics in tight sands could be responsible of unexpected behaviors of some fields.

Several tight sands are dual porosity rocks with a fraction of the void space conformed by secondary pores and the other by the flow paths (inter-granular slots) among these pores. Other tight sands have been observed as a pore structure, where the void space consist of primary inter-granular porosity propped open by contact points between individual rounded quartz sand grains (See Figure 1). ${ }^{14,15}$

(a)

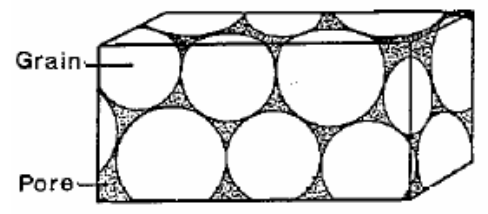

(b)

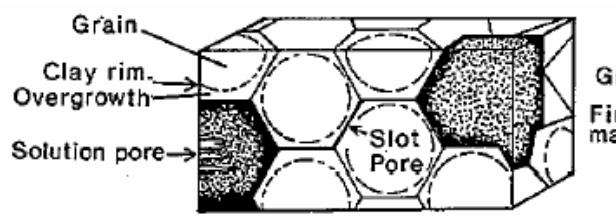

(c)

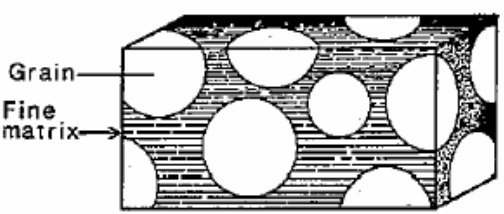

Figure 1. Three main types of pore geometry of tight sand-stones. (a) Grain supported pores, (b) Slot and solution pores, (c) Matrix supported grains. Taken from Ref. 15.

Reports confirm that most common tight sands have slit-like or sheet-like pore types as primary porosity. ${ }^{16-19}$ Thin sections (See Figure 2) have shown that a network of polyhedral sheets is strongly related to pore size distribution for most of tight sands of potential commercial interest and this 
structure controls the permeability to gases for this low permeability media. ${ }^{15}$ Very small characteristic sized for the flow path openings in tight sands are widely reported. Widths have been in the range from 0.03 to $0.27 \mu \mathrm{m}^{17}$ which include other realm of sizes reported. ${ }^{16,20}$ Surface areas measured by nitrogen adsorption are typically about 200 times greater than sheet-type pore areas existent which is reported as the controller of permeability. ${ }^{18}$ Accordingly, the surface area of the polyhedral structure is only a small fraction of the total surface area of the system and the surface area appears to be dominated by the matrix composed by significantly fine particles, such as clays, cherts and others micro-porous minerals.

(a)

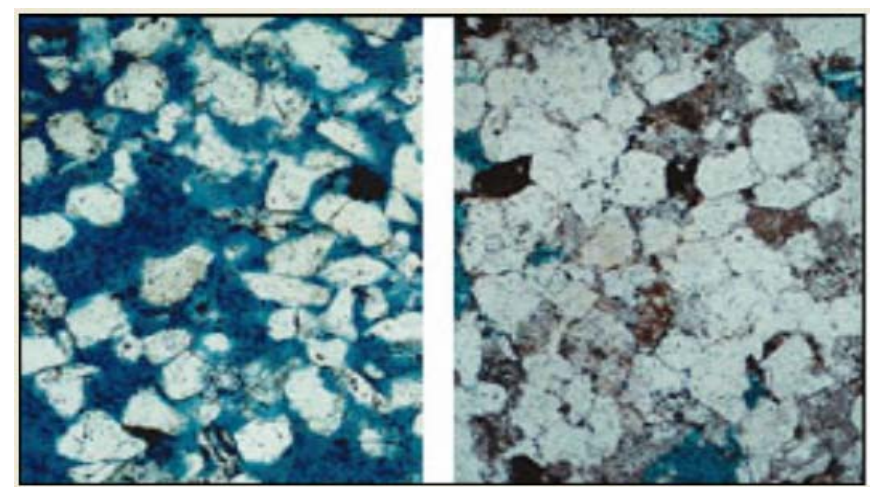

Figure 2. Microscopic sections of (a) Conventional, and (b) Tight sandstone. Conventional sandstone has well- connected pores (dark blue). Tight gas sandstone pores are irregularly distributed and weakly connected. Taken from www.netl.doe.gov.

A term named as the pore quality was defined as a value based on the degree of porosity occlusion, going from 0 (a solid mineral grain) to 1 (a completely empty pore). Thus, a rock with high quality porosity should have a pore quality near 1 and rocks with several occlusions of pore spaces (fine structured minerals as clays, cherts) will have pore quality closer to 0.25 . The reported range of pore quality between 0.26 and 0.45 for tight sands means an abundance of clays and other forms of microporosity. ${ }^{21}$ For the Bossier tight sands effective porosities from $1 \%$ to $17 \%$, and permeability from 0.001 to $1 \mathrm{mD}$ are reported. Permeabilities lower than $0.001 \mathrm{mD}$ are associated here to nonreservoir and seal rocks. ${ }^{22}$

The unconventional characteristics of these low permeability porous media have not been considered an impediment to use conventional reservoir simulators for predicting of tight sand reservoiors. ${ }^{23-25}$ 
Some authors focused on improving the physical and mathematical description of these unusual systems in order to explain the unexpected results reported. ${ }^{26-28}$

A commonly accepted approach modified certain aspects of conventional reservoir simulators known by BOAST (Black Oil Applied Simulation Tool). ${ }^{29}$ The modification included the use of PengRobinson equation to calculate the gas formation (release) factor and the properties of the gas. Phase transitions allowed the gas to be in liquid phase. In addition a dependence of permeability with pressure was introduced though an empirical equation developed by McKee. The effective permeability of each block was estimated by performing a weighted average of the width of the fracture and the width of the rock matrix. This simulator was used in several sensibility studies of flow in reservoirs. ${ }^{30}$

Efforts were made on the formulation of "dual porosities" and dual permeabilities simulators considering that many reservoirs are naturally fractured. Discrete network models are more adequate than Dual porosity models when fluid flow towards production wells is dominated by flow through fractures. Significant differences on flow were observed when single porosity and dual porosity were used. $^{31}$

The effects of fracture concentration, capillary pressure in the fractures and lenticularity on the fluid flow were also studied. ${ }^{32,33}$ The simulator used assumes that the fracture plane coincides with two of the three Cartesian axes. The model treats a fractured cellblock as being naturally fractured along any two of the principal axes. Traditional treatment (Darcy flow) of the fluid flow equations was performed for both water and gas. The authors use the effective permeability and average porosity concepts for each cell block but the effective permeability was calculated in such a way that it was dependent of the cellblock dimension.

Following along the same line of thought, others focused their efforts on obtaining the permeability tensor by creating a "realistic" fracture network model from outcrops and core data and incorporating this network into the reservoir simulator. ${ }^{34-36}$ It should be noted that the interaction between the fracture network and the rock matrix is poorly understood at the reservoir length scale. The traditional uncertainty surrounding the geometry of fractures and their dynamic behavior can now be added to the uncertainty surrounding the appropriateness of the fluid flow equations that are used.

Most of reduction in permeability due to stresses occurs in sandstones with the lowest values of porosity and permeability. The declining rate of permeability with stress is highly variable and consequently reservoir exploitation parameters such as abandonment pressure are variable. ${ }^{16}$ The 
impact on production rate and cumulative of this, was evaluated through a simulator that incorporates pore pressure dependent transmissibility. ${ }^{37}$

The effect of the, usually large, amount of water pumped during hydraulic fracturing on well productivity is also poorly understood. The location of water in the formation is influenced by geomechanical effects, particularly by stress-dependent permeability. In order to investigate water blockage or formation damage a combined reservoir/geomechanics/fracturing model has been developed. $^{39}$ The model simulates the dynamic fracture evolution during pumping, stress dependent reservoir permeability during injection and production, and stress dependent propped fracture conductivity.

A dual mechanistic approach that contains the dispersive and the convective contribution to the flow was other of the partially valid simulator developed for tight sand gas reservoirs. ${ }^{4}$ The idea behind this alternative is reasonable, but important limitations of their formalism are listed bellow:

- The micro-pores were considered accessible only to gas, whereas the water primarily resides in the macro-pores. However, the distribution of fluids is expected highly different in tight sands since water should be the wetting phase.

- It is said that when a pressure gradient is imposed, the thermodynamic equilibrium is distorted between the gas that is in solution in the water and the gas that is present in the micro-pores, creating local concentration gradients. Following our line of thought, gas should be driven into the macro-pores by diffusion. However, if water is the wetting phase, with gas in the small pores and water in the large pores, the system is not in thermodynamic equilibrium, even before of the imposed pressure gradient, since capillary pressure would favor the flow of the gas towards the large pores.

- The equations are supposed to capture the slippage effect through a dispersive term that explicitly appears in the equation. However, a huge misunderstanding of the contribution of ordinary diffusion and Knudsen diffusion is evidenced when the Klinkenberg parameter $b$ is obtained as a function of the ordinary diffusion coefficient.

Also, none of the developed or adapted simulator meets all the aspects relevant for predict the behavior of tight sands reservoirs, but an analysis of then allow identifying the amount and type of phenomena that a model to simulate the performance of this kind of reservoirs should have.

In the next sub-section we formulate a multi-mechanistic, dual porosity and dual permeability approach, that overcome previous deficiencies, in order to evaluate the need of considering all these phenomena in the formulation to reach reliable forecasts of tight sands gas reservoir. 


\subsubsection{Multi-Mechanistic Approach.}

\section{MASS CONSERVATION EQUATIONS}

The porous medium was modeled using a dual-porosity and dual-permeability approach. The mass balance of methane, water and salt molecules contained in gas and in liquid phase is formulated by two equations, one for the porous matrix and other for the fractured regions. Thus a system of six equations will determine the flow dynamic. The assumption of local phase equilibrium allows working with the mass conservation equation of each component in all phases, without considering a transfer term among different phases.

In the case of methane in the matrix, the transport in both liquid and gas phases can be written:

$$
\begin{aligned}
\frac{\partial}{\partial t}\left[\phi \rho_{g}^{s c}\left(\frac{S_{g}}{B_{g}}+\frac{S_{l} R_{S l}}{B_{l}}\right)\right] & =-\vec{\nabla} \cdot\left(\vec{v}_{g} \frac{\rho_{g}^{s c}}{B_{g}}+\vec{v}_{l} \frac{\rho_{g}^{s c} R_{S l}}{B_{l}}\right)+ \\
+ & \vec{\nabla} \cdot\left[\phi \rho_{g}^{s c}\left(\frac{S_{g}}{B_{g}} D_{m g}^{*} \frac{1}{x_{m g}} \vec{\nabla} x_{m g}+\frac{S_{l} R_{S l}}{B_{l}} D_{m l}^{*} \frac{1}{x_{m l}} \vec{\nabla} x_{m l}\right)\right]+\frac{q_{m}}{V_{T}}-T_{m}^{M-F}
\end{aligned}
$$

This advection-diffusion equation states that local variation of total mass (in liquid and gas) of methane per unit of total volume is equal to the sum of mass inputs due to convection, diffusion, external sources and transfer between matrix and fracture. The equation is written in terms of parameters conventionally used in the oil-gas engineering argot. The physical meanings of these parameters are detailed, below, in this section.

Darcy velocities in liquid and gas phases are given by

$$
\vec{v}_{k}=-\frac{K_{k} k r_{k}}{\mu_{k}} \vec{\nabla}\left(P_{k}+\rho_{k} g z\right) ; k=l, g
$$

In a more compact form, equation (1) can be rewritten as

$$
\frac{\partial F_{k}}{\partial t}=\vec{\nabla} \cdot\left(M C_{k g} \vec{\nabla}\left(P_{g}+\rho_{g} g z\right)+M C_{k l} \vec{\nabla}\left(P_{l}+\rho_{l} g z\right)+M D_{k g} \vec{\nabla} x_{k g}+M D_{k l} \vec{\nabla} x_{k l}\right)+\frac{q_{k}}{V_{T}}-T_{k}^{M-F}
$$

For $k=m$, the introduced parameters are

$$
F_{m}=\phi \rho_{g}^{s c}\left(\frac{S_{g}}{B_{g}}+\frac{S_{l} R_{S l}}{B_{l}}\right)=\frac{m_{s}}{V_{T}}
$$




$$
\begin{aligned}
& M C_{m g}=\frac{K_{g} k r_{g}}{\mu_{g}} \frac{\rho_{g}^{s c}}{B_{g}} ; M C_{m l}=\frac{K_{l} k r_{l}}{\mu_{l}} \frac{\rho_{g}^{s c} R_{S l}}{B_{l}} ; \\
& M D_{m g}=\frac{\phi S_{g} D_{m g}^{*}}{x_{m g}} \frac{\rho_{g}^{s c}}{B_{g}} ; M D_{m l}=\frac{\phi S_{l} D_{m l}^{*}}{x_{m l}} \frac{\rho_{g}^{s c} R_{S l}}{B_{l}} .
\end{aligned}
$$

For $k=w$, the parameters involved are

$$
\begin{aligned}
& F_{w}=\phi\left(\frac{S_{g} \rho_{w}^{s c} R_{S g}}{B_{g}}+\frac{S_{l} \rho_{l}^{s c}\left(1-\omega_{s}\right)}{B_{l}}\right)=\frac{m_{w}}{V_{T}} ; \\
& M C_{w g}=\frac{K_{g} k r_{g}}{\mu_{g}} \frac{\rho_{w}^{s c} R_{S g}}{B_{g}} ; M C_{w l}=\frac{K_{l} k r_{l}}{\mu_{l}} \frac{\rho_{l}^{s c}\left(1-\omega_{s}\right)}{B_{l}} ; \\
& M D_{w g}=\frac{\phi S_{g} D_{w g}^{*}}{x_{w g}} \frac{\rho_{w}^{s c} R_{S g}}{B_{g}} ; M D_{w l}=\frac{\phi S_{l} D_{w l}^{*}}{x_{w l}} \frac{\rho_{l}^{s c}\left(1-\omega_{s}\right)}{B_{l}} .
\end{aligned}
$$

Equation (3) also rules the transport of salt in matrix ( $k=s$ ), with the particularity that there is not salt in gas phase $\left(x_{s g}=0\right)$ but in solid phase. Here, the involved parameters are

$$
\begin{aligned}
& F_{s}=\frac{\phi S_{l} \rho_{l}^{s c} \omega_{s}}{B_{l}}+\frac{N_{s s} M m_{s}}{V_{T}}=\frac{m_{s}}{V_{T}} ; \\
& M C_{s g}=0 ; M C_{s l}=\frac{K_{l} k r_{l}}{\mu_{l}} \frac{\rho_{l}^{s c} \omega_{s}}{B_{l}} ; \\
& M D_{s g}=\text { bounded; } M D_{s l}=\frac{\phi S_{l} D_{s l}^{*}}{x_{s l}} \frac{\rho_{l}^{s c} \omega_{s}}{B_{l}} .
\end{aligned}
$$

There are other similar equations to equations (3) to (10) for the fracture system. In the case of the transport equation (3), the sign of the transfer term changes, so that

$$
\begin{aligned}
& F_{m}=\phi \rho_{g}^{s c}\left(\frac{S_{g}}{B_{g}}+\frac{S_{l} R_{S l}}{B_{l}}\right)=\frac{m_{s}}{V_{T}} ; \\
& M C_{m g}=\frac{K_{g} k r_{g}}{\mu_{g}} \frac{\rho_{g}^{s c}}{B_{g}} ; M C_{m l}=\frac{K_{l} k r_{l}}{\mu_{l}} \frac{\rho_{g}^{s c} R_{S l}}{B_{l}} ;
\end{aligned}
$$




$$
\frac{\partial F_{k}}{\partial t}=\vec{\nabla} \cdot\left(M C_{k g} \vec{\nabla}\left(P_{g}+\rho_{g} g z\right)+M C_{k l} \vec{\nabla}\left(P_{l}+\rho_{l} g z\right)+M D_{k g} \vec{\nabla} x_{k g}+M D_{k l} \vec{\nabla} x_{k l}\right)+\frac{q_{k}}{V_{T}}+T_{k}^{M-F}
$$

In order to get simpler notation, we are not using the superscript $M$ and $F$ in the parameters contained in equations (3) and (15), respectively.

Finally, there is a system of six differential equations to solve, three equations (3) for the matrix with $k=m, w, s$ and three equations (15) for the fractures.

\section{MATRIX-FRACTURE TRANSFER TERM}

The mass transfer term between matrix and fractures is assumed here analogous to the one introduced previous works ${ }^{38}$ but considering both convective and diffusive transport, i.e.

$$
\begin{aligned}
& T_{k}^{M-F}=-T_{k}^{F-M}= \\
& \quad=\left(\frac{8}{l^{2}}\right)\left(M C_{k g}^{M}\left(P_{g}^{M}-P_{g}^{F}\right)+M C_{k l}^{M}\left(P_{l}^{M}-P_{l}^{F}\right)+M D_{k g}^{M}\left(x_{k g}^{M}-x_{k g}^{F}\right)+M D_{k l}^{M}\left(x_{k l}^{M}-x_{k l}^{F}\right)\right)
\end{aligned}
$$

where $k=m, w, s$. The geometrical factor $\frac{8}{l^{2}}$ contain the fracture spacing $l$, as in previous works. ${ }^{38}$

\section{FROM OIL-NOTATION TO PHYSICAL PARAMETERS}

Equations (4), (9) and (10) contain oil industry parameters, which are volumetric ratios after a flash depressurization, as shown on Figure 3.

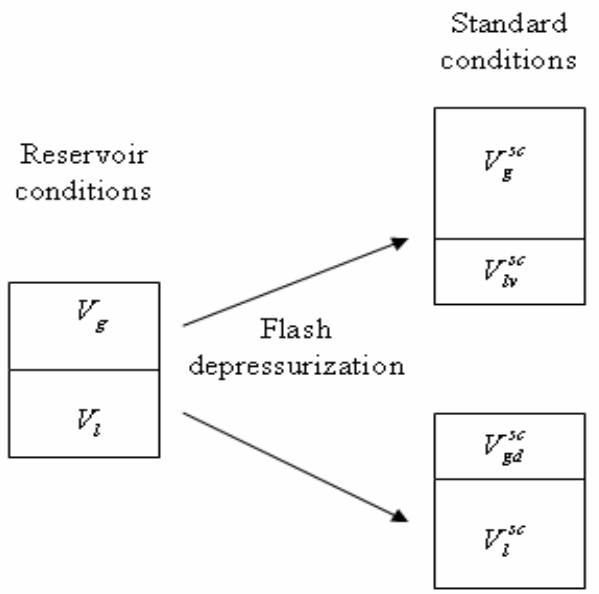

Figure 3. Representations of the phase volumes at reservoir conditions and at standard conditions after a flash depressurization of each phase separately. 
Assuming that at standard conditions, there is neither methane dissolved in liquid nor water vaporized, the volumetric ratios are

$$
\begin{aligned}
& B_{g}=\frac{V_{g}}{V_{g}^{s c}}=\frac{\rho_{g}^{s c} M m_{g}}{\rho_{g} M m_{m} x_{m g}} ; \\
& B_{l}=\frac{V_{l}}{V_{l}^{s c}}=\frac{\rho_{l}^{s c} M m_{l}}{\rho_{l}\left(M m_{w} x_{w l}+M m_{s} x_{s l}\right)}=\frac{\rho_{l}^{s c} M m_{l} \omega_{s}}{\rho_{l} M m_{s} x_{s l}} ; \\
& R_{S g}=\frac{V_{l v}^{s c}}{V_{g}^{s c}}=\frac{\rho_{g}^{s c} M m_{w} x_{w l}}{\rho_{w}^{s c} M m_{m} x_{m l}} ; \\
& R_{S l}=\frac{V_{g d}^{s c}}{V_{l}^{s c}}=\frac{\rho_{l}^{s c} M m_{m} x_{m l}}{\rho_{g}^{s c}\left(M m_{w} x_{w l}+M m_{s} x_{s l}\right)}=\frac{\rho_{l}^{s c} M m_{m} x_{m l} \omega_{s}}{\rho_{g}^{s c} M m_{s} x_{s l}} .
\end{aligned}
$$

Here it is introduced the mass fraction of salt in depressurized liquid

$$
\omega_{s}=\frac{M m_{s} x_{s l}}{M m_{w} x_{w l}+M m_{s} x_{s l}} .
$$

Notice that by substituting relations from (17) to (21) in the expressions (4), (7) and (10), the parameters $F_{k}$ are reduced to mass of the component $k$ in all the phases per unit of total volume.

\section{PVT EQUILIBRIUM}

The system composed by methane, water and salt has up to three phases, which are assumed in local equilibrium. The computation of the variables that characterize of the equilibrium state can be performed by using cubic equations of state (EOS), as suggested for example on fig. 5.1 in Ref.40 Several types of cubic EOS, such as the modified Patel-Teja EOS $^{41,42}$ and its Valderrama modification, ${ }^{43,44}$ and mixing rules that can be fitted to the specific system methane-water-salt, could be in principle implemented. However, previous work faced difficulty to find all fitted parameters of the EOS as well as to know the fugacity coefficient formula used.

The algorithm implemented to find the equilibrium state of the system is as follows. First, a function to compute the parameters $x_{w g}, x_{m l}, x_{s l}$ and $N_{s s}$, given the total number of moles of each component $N_{m}, N_{w}$ and $N_{s}$, as well as $P_{g}$ and $T$, is programmed. It start from a first guess that the methane is in the gas phase and the water and the salt in the liquid phase, i.e., 


$$
x_{w g}=0, x_{m l}=0, x_{s l}=\frac{N_{s}}{N_{s}+N_{w}} \text { and } N_{s s}=0 \text {. }
$$

Then, $\omega_{s}$ is found from equation (21) and limited to the upper value $\omega_{s}^{\max }=\omega_{s}^{\max }(T)$ described in the PVT correlations section. The amount of vaporized water $x_{w l}=x_{w l}\left(P_{g}, T, \omega_{s}\right)$ and the dissolved methane $x_{m l}=x_{m l}\left(P_{g}, T, x_{m g}, \omega_{s}\right)$ are recalculated from the correlations also detailed in the PVT correlations section. After obtaining

$$
\begin{aligned}
& x_{m g}=1-x_{w g}, \\
& x_{w l}=1-x_{m l}-x_{s l} ;
\end{aligned}
$$

the number of moles in gas and liquid phases are

$$
\begin{aligned}
& N_{T g}=\frac{x_{m l} N_{w T}-x_{w l} N_{m T}}{x_{m l} x_{w g}-x_{w l} x_{m g}}, \\
& N_{T l}=-\frac{x_{m g} N_{w T}-x_{w g} N_{m T}}{x_{m l} x_{w g}-x_{w l} x_{m g}} .
\end{aligned}
$$

Thus, a new value of $x_{s l}$ arises from

$$
x_{s l}=\frac{N_{s}}{N_{T l}},
$$

where $\omega_{s}$ is found again from equation (21) and compared with the upper limiting value. In case that $\omega_{s}>\omega_{s}^{\max }$, then $\omega_{s}=\omega_{s}^{\max }$ and it is substituted in

$$
x_{s l}=\frac{\omega_{s} M m_{w}\left(1-x_{m l}\right)}{M m_{s}+\omega_{s}\left(M m_{w}-M m_{s}\right)}
$$

This equation comes up from combining (21) and (24), and solving for $x_{s l}$. From the new value $x_{s l}$ found, a new iteration start until convergence is reached. The convergence is accelerated by using a Newton-Rapson method, though.

Once the parameters $x_{w g}, x_{m g}, x_{m l}, x_{w l}, x_{s l}$ and $\omega_{s}$ are determined, the phase densities $\rho_{g}=\rho_{g}\left(P, T, x_{w g}\right)$ and $\rho_{l}=\rho_{l}\left(P, T, x_{m l}, x_{s l}\right)$ are obtained from the correlations described in the PVT correlations section. Moreover the following parameters are computed 


$$
\begin{aligned}
& N_{T s}=N_{s s}=N_{s T}-x_{s l} N_{T l} . \\
& M m_{g}=M m_{m} x_{m g}+M m_{w} x_{w g} . \\
& M m_{l}=M m_{m} x_{m l}+M m_{w} x_{w l}+M m_{s} x_{s l} . \\
& V_{i}=\frac{N_{T i} M m_{i}}{\rho_{i}}, \text { for } i=g, l, s . \\
& S_{i}=\frac{V_{i}}{V_{g}+V_{l}+V_{s}}, \text { for } i=g, l .
\end{aligned}
$$

Notice that the way of computing the fluid phase saturation from (33) implies that $S_{g}+S_{l}<1$ when there is salt deposition ( $N_{s s}>0$ ). So, the salt deposition would cause a reduction in the fluid saturations and therefore in the relative permeabilities. This approach is slightly different one previously reported, ${ }^{45}$ which considers a reduction on porosity and permeability due to the salt deposition.

The described function that gives the equilibrium state of the system given $N_{m}, N_{w}, N_{s}, P_{g}$ and $T$, can not be used directly in our case when our set of known parameters are $N_{m}, N_{w}, N_{s}, \phi V_{T}$ and $T$, or just $n_{m}, n_{w}, n_{s}$ and $T$. Thus, it is necessary to call the described function iteratively until finding the gas pressure $P_{g}$ that satisfies the right pore volume $\phi V_{T}=V_{g}+V_{l}+V_{s}$.

\section{PETROPHYSiCAL CORRELATIONS}

\section{Gas permeability}

The gas permeability is obtained from the Klinkenberg relation: ${ }^{12}$

$$
K_{g}=K_{\infty}\left(1+\frac{b}{P_{g}}\right) .
$$

The Klinkenberg permeability $\left(K_{\infty}\right)$ is usually measured by using a non-polar liquid. The Klinkenberg parameter $(b)$ can be also determined experimentally by measuring gas permeability at several pressures. Klinkenberg found, in his original experiments, that the Klinkenberg parameter $b$, has some dependence with pressure, fact that was confirmed in recent experiments. ${ }^{13}$ We will go deeper to this point in next section. 
If there is no experimental data available, the parameter $b$ can be estimated from the Klinkenberg permeability according to the correlation reported: ${ }^{16}$

$$
\frac{b}{\mathrm{~atm}}=0.86\left(\frac{K_{\infty}}{\mathrm{mD}}\right)^{-0.33} \text {. }
$$

Here they are introduced the field units for pressure $(1 \mathrm{~atm}=101325.01 \mathrm{~Pa})$ and permeability $(1 \mathrm{mD}$ $=0.9869233 \mathrm{e}-15 \mathrm{~m}^{2}$ ).

Other published correlation that involves more parameters is, ${ }^{20}$

$$
\frac{b}{\mathrm{~atm}}=0.0955 \times 10^{6}\left(\frac{K_{\infty}}{\mathrm{mD}} \frac{1}{\phi S_{g}}\right)^{-0.53}
$$

Klinkenberg parameter $(b)$ and the Knudsen diffusion coefficient $\left(D^{K}\right)$ are related by: ${ }^{46}$

$$
b=\frac{\mu_{g}}{K_{\infty}} D^{K} .
$$

Data available from air (Nitrogen) flow in core samples (shown on Figure 4) can be fitted from relation with the form

$$
D^{K}=185525 \frac{\mathrm{m}^{2}}{s}\left(\frac{K_{\infty}}{\mathrm{m}^{2}}\right)^{0.7014} .
$$

The Knudsen coefficient changes with the molecular mass of the gas according to:

$$
\frac{D_{1}^{K}}{D_{2}^{K}}=\sqrt{\frac{M m_{g 2}}{M m_{g 1}}} .
$$




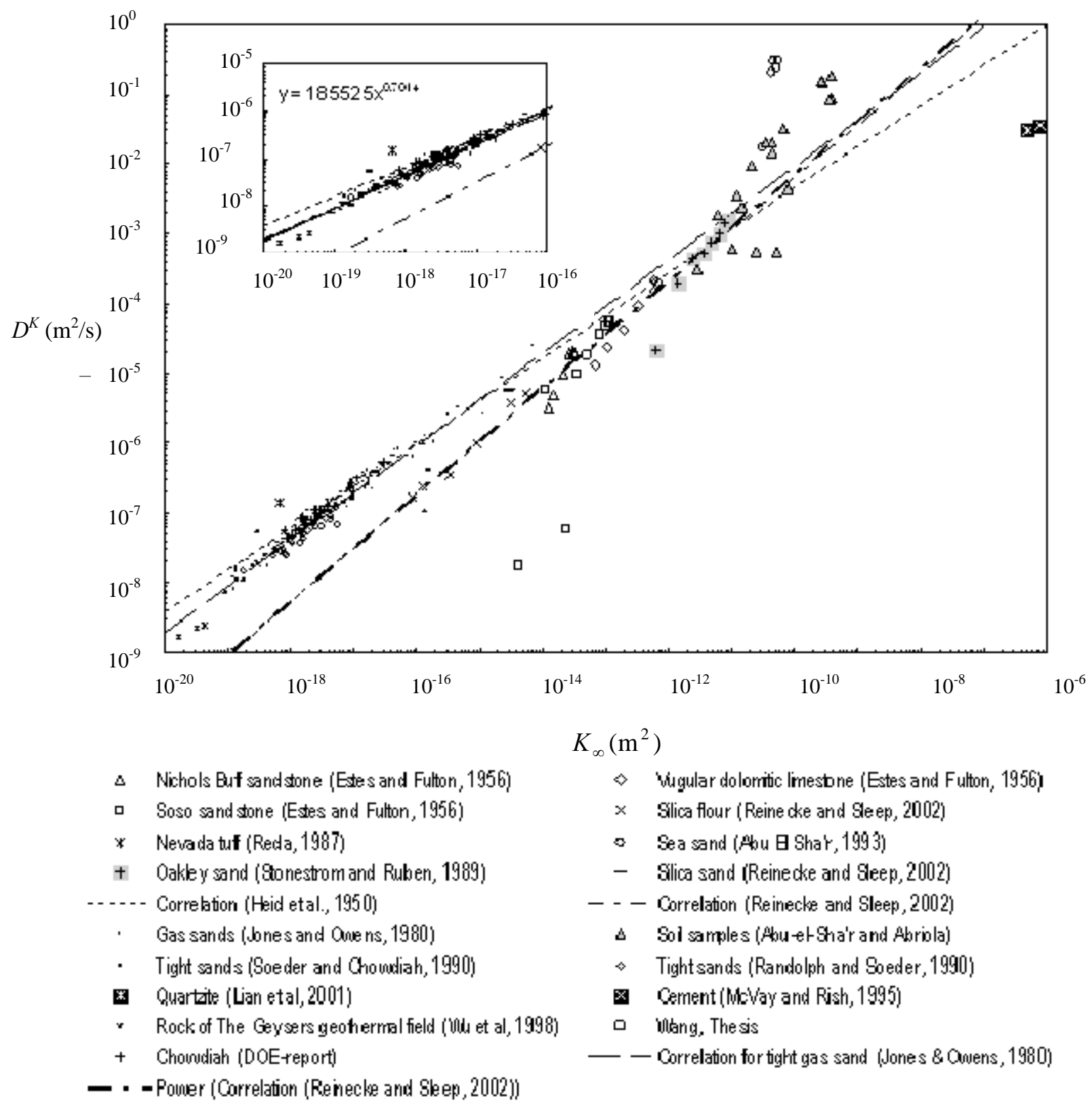

Figure 4. Plot of the Klinkenberg permeability versus the Knudsen diffusion coefficient for air (nitrogen) flow. Points are experimental results and the lines are available correlations, including the solid line corresponding to equation (38).

Therefore, from relations (37) to (39) the Klinkenberg parameter satisfies regardless the gas composition that 


$$
b=b_{0}\left(\frac{K_{\infty}}{K_{\infty 0}}\right)^{-0.33},
$$

which is the same functional dependence (35).

By using the point $\left(b_{0}, K_{\infty 0}\right)=\left(6 \times 10^{5} \mathrm{~Pa}, 2.37 \times 10^{-17} \mathrm{~m}^{2}\right)$ obtained from data in Ref.13 for methane gas as $P \rightarrow 0$, it gives

$$
\frac{b}{\mathrm{~atm}}=1.73\left(\frac{K_{\infty}}{\mathrm{mD}}\right)^{-0.33} \text {. }
$$

In summary, correlations (35), (36) and (41) could be used for the Klinkenberg parameter.

\section{Liquid permeability}

The liquid permeability is different from the Klinkenberg permeability because of the electro-kinetic effects. A correlation obtained in previous work is: ${ }^{16}$

$$
\frac{K_{l}}{\mathrm{mD}}=\left(\frac{K_{\infty}}{\mathrm{mD}}\right)^{1.32} .
$$

\section{Relative Permeability}

Relative permeabilities are obtained by knowing the "end points" for each phase $k$, which are the relative permeability values $\left(k r_{k i}\right)$ at the irreducible saturation of the other phase. The simplest relation is a power law, which for the phase $k$ has the form

$$
k r_{k}=k r_{k i} S_{r k} n_{k}
$$

where the reduced saturation of the phase $k$ is

$$
S_{r k}=\frac{S_{k}-S_{k i}}{1-S_{g i}-S_{l i}} .
$$

The well known Brooks-Corey-Burdine (BCB) correlation $^{47}$ is extended to satisfy the end points for the wetting and non wetting phase in the form ${ }^{48}$

$$
k r_{w}=k r_{w i} S_{r w}^{\frac{2+3 \xi}{\xi}}
$$




$$
k r_{n w}=k r_{n w i}\left(1-S_{r w}\right)^{2}\left(1-S_{r w} \frac{2+\xi}{\xi}\right) .
$$

Here $\xi$ is the sorting factor and reduced saturation of the wetting phase $\left(S_{r w}\right)$ is obtained from equation (44).

\section{Capillary pressure}

The capillary pressure $\left(P_{c}\right)$ is defined as the excess of pressure at the non-wetting phase $\left(P_{n w}\right)$ regarding the pressure of the wetting phase $\left(P_{w}\right)$, i.e.

$$
P_{n w}=P_{w}+P_{c}
$$

The BCB correlation computes originally the capillary pressure in a displacement process from the displacement pressure $\left(P_{d}\right)$ and the sorting factor $(\xi)$. It can be extended to satisfy the end points of the relative permeability by using $S_{r w}$ from (44) as

$$
P_{c}=P_{d} S_{r w}^{-\frac{1}{\xi}}
$$

Besides the BCB correlation (48), the capillary pressure can be estimated by interpolation from the tabulated values reported for Coastal, Paludal and Paralic samples. ${ }^{33}$

\section{Diffusion-dispersion coefficient}

The dispersion coefficient, $D^{*}$, is a tensor usually represented by its components in the flow direction (the longitudinal dispersion coefficient, $D_{L}$ ) and perpendicular to flow, (the transversal dispersion coefficient, $D_{T}$ ). As can be seen in Figure 5, five different regimes of dispersion are recognized from the dependence among $D_{L}$ and $D_{T}$ with the Péclet number $\left(P e=l \mathrm{v} / D_{m}\right) .{ }^{49-51}$ In the dispersion regime where $P e \leq 0.3$, the diffusion dominates the dispersive transport and the coefficients $D_{L}$ and $D_{T}$ are related with the molecular diffusion coefficient, $D_{m}=D_{12}=D_{21}$, through:

$$
\frac{D^{*}}{D_{m}}=\frac{D_{L}}{D_{m}}=\frac{D_{T}}{D_{m}}=\frac{1}{\tau} .
$$

Here $\tau$ is the porous medium's tortuosity, defined as the tortuous trajectory length between two endpoints of the porous media divided by the straight distance between the same two points. It has been reported that depending of the porous medium, $1 / \tau$ varies from $1 / 8$ to $2 / 3$. Estimated tight sands totuosity values have been also in this range. ${ }^{15}$ 
Thus, here the dispersion coefficients (for $\mathrm{Pe}<0.3$ ) are given by:

$$
D_{k i}^{*}=\frac{D_{k i}}{\tau}, \text { with } i=g, l \text { and } k=m, w, s
$$

The diffusivities coefficients were considered to comply the Onsager relation, through which $D_{m g}=D_{w g}$ and $D_{w l}=D_{m l}$. The self-diffusion of methane ${ }^{52}$ is used to compute $D_{m g}$ and the aqueous diffusion coefficient of methane ${ }^{53}$ was used to estimate $D_{m l}$.

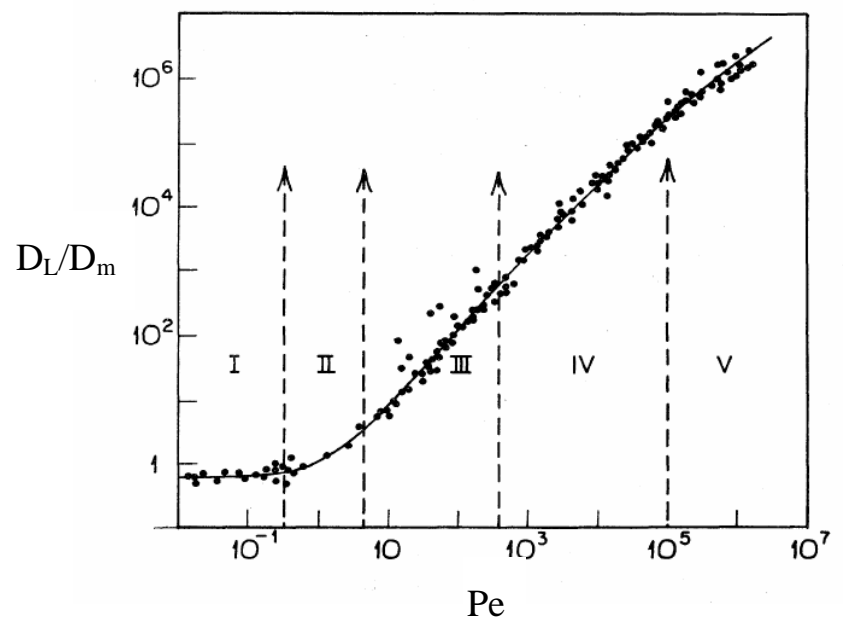

Figure 5. Experimental data for longitudinal dispersion coefficient $\mathrm{D}_{\mathrm{L}}$ vs Peclet numbers Pe ( $=d_{g} v / \mathrm{D}_{\mathrm{m}}$, with $d_{g}$ as the average diameter of a grain, $v$, velocity and $\mathrm{D}_{\mathrm{m}}$, molecular diffusivity) in five different regimes. $D_{m}$ is molecular diffusivity. Taken from Ref. 51

\section{PVT CORRELATIONS}

\section{Constant parameters}

Constant parameter, that characterize each component, used in the following PVT correlations are listed in Table 1. Other constant parameters that characterize the standard condition state $\left(T^{s c}=60 \mathrm{~F}\right.$ $=288.70 \mathrm{~K}$ and $P^{s c}=1 \mathrm{~atm}=101325.01 \mathrm{~Pa}$ ) are the densities $\rho_{g}^{s c}=0.6772 \mathrm{~kg} / \mathrm{m}^{3}$ and $\rho_{w}^{s c}=$ $998.01 \mathrm{~kg} / \mathrm{m}^{3}$, which are obtained from the density correlations described below. 


\begin{tabular}{ccccccc}
\hline \hline Component & $M m(\mathrm{~g} / \mathrm{mol})$ & $T_{c}(\mathrm{~K})$ & $P_{c}(\mathrm{MPa})$ & $Z_{c}$ & $T_{f}(\mathrm{~K})$ & $\omega$ \\
\hline Methane & 16.04276 & 190.564 & 4.599 & 0.2862 & 273.15 & 0.0115 \\
Water & 18.01528 & 647.14 & 22.055 & & & \\
Salt (NaCl) & 58.4428 & & & & & \\
\hline \hline
\end{tabular}

Table 1. Each component's characteristic parameters. Blank boxes correspond to unused parameters.

\section{Gas density}

In general, $\rho_{g}=\rho_{g}\left(P, T, x_{w g}\right)$. The real gas equation of state is used to obtain the gas density as

$$
\rho_{g}=\frac{M m_{g} P_{g}}{Z R_{g} T} .
$$

Correlation for the compressibility factor $(Z)$ of natural gases is obtained from the Starling modification of Benedict-Webb-Rubin EOS $^{54}$ as described in Ref.40 This correlation for $Z$ is applied under the assumption that the gas is pure methane $\left(x_{w g}=0\right)$.

\section{Liquid density}

In general, $\rho_{l}=\rho_{l}\left(P, T, x_{m l}, x_{s l}\right)$. A reported correlation, ${ }^{55,56}$ is used to compute the liquid density as a function of pressure, temperature and salt content $\rho_{l}=\rho_{l}\left(P, T, \omega_{s}\right)$. This relation does not consider the methane dissolved in liquid, though. It assumes $x_{m}=0$.

\section{Water saturation pressure}

The saturation pressure of pure water $\left(P_{s w}\right)$ is used to compute the Henry constant for methane dissolved in water $\left(H_{m}^{0}\right)$, the maximum amount of salt dissolved in liquid $\left(\omega_{s}\right)$, the amount of dissolved methane $\left(x_{m l}\right)$ and vaporized water $\left(x_{w g}\right)$. This parameter is only a function of the temperature and was estimated from the correlation. ${ }^{57,40}$

\section{Amount of vaporized water}

The amount of vaporized water $\left(x_{w g}\right)$ is computed from the Raoult's Law: ${ }^{40}$

$$
x_{w g}=x_{w g}\left(P_{g}, T, \omega_{s}\right)=\frac{P_{s w}}{P_{g}} \varphi_{y s} .
$$


The additional factor $\varphi_{y s}$ in equation (52) appears as a correction for the salt content in liquid. The Katz correction in form of graph was later expressed as a second degree polynomial in the salt weight fraction $\varphi_{y s}=\varphi_{y s}\left(\omega_{s}\right) \cdot{ }^{58,40}$ The computed value of $x_{w g}$ from equation (52) is restricted by the condition $x_{w g} \leq 1$.

It should be mentioned that this simple procedure to obtain the vaporized water usually underpredict the reported values, as it was realized from Fig. 2.26 in Ref.40, as well as other reported figures. ${ }^{59,60}$

\section{Amount of dissolved methane}

The amount of dissolved methane is found by using the Henry's Law ${ }^{40}$

$$
x_{m l}=x_{m l}\left(P_{g}, T, x_{m g}, \omega_{s}\right)=\frac{H_{m}}{P_{g}} x_{m g} \varphi_{x s} .
$$

The additional factor $\varphi_{x s}$ is a correction for the salt content in liquid as proposed originally by McKetta and Wehe. The original graphic relation was presented ${ }^{58}$ in form of an analytical expression $\varphi_{x s}=\varphi_{x s}\left(T, \omega_{s}\right){ }^{40}$

Notice that equation (53) is a simplified version of the more general Henry's Law, that assumes the fugacity in the gas phase equal to the gas pressure, which is strictly valid ideal gases $(Z=1)$. Computing the fugacity increases the computing time.

The Henry constant for methane $\left(H_{m}\right)$ in equation (53) is a function of pressure and temperature according to ${ }^{40}$

$$
H_{m}=H_{m}\left(P_{g}, T\right)=H_{m}^{0} \exp \left(v_{m}^{\infty} \frac{P_{g}-P^{0}}{R_{g} T}\right),
$$

where the reference pressure $P^{0}=P^{s c}$ and molar volume is considered as $v_{m}^{\infty}=40 \times 10^{-6} \mathrm{~m}^{3} / \mathrm{mol}^{40}$ Moreover, the Henry constant at standard pressure $\left(H_{m}^{0}\right)$ is obtained from the correlation for methane dissolved in water $H_{m}^{0}=H_{m}^{0}\left(P_{s w}(T), T\right){ }^{61}$

\section{Gas viscosity}

In general, $\mu_{g}=\mu_{g}\left(P, T, y_{w}\right)$. A correlation proposed ${ }^{62,40}$ finds the viscosity of natural gases as a function of their molecular mass, density and the temperature, i.e., $\mu_{g}=\mu_{g}\left(M m_{g}, \rho_{g}, T\right)$. In this 
case, this correlation is applied under the assumption that the molecular mass of the gas is the one of methane $\left(M m_{g}=M m_{m}\right)$.

\section{Maximum amount of salt dissolved in liquid}

The maximum amount of salt that can be dissolved in liquid at a certain temperature was estimated by using the correlation for a brined system reported. ${ }^{63}$ This relation does not consider the effect of the methane dissolved in liquid, which reduces the amount of the dissolved salt (see Ref. 41). The maximum amount of salt in liquid is quantified by the weight fraction in depressurized liquid $\left(\omega_{s}^{\max }\right)$, which is shown on Figure 6. Notice that the phrase "depressurized liquid" refers to the way that the weight fraction is calculated in equation (21) by ignoring the dissolved methane. Of course, a decreasing in pressure may produce a partial deposition of the dissolved salt. ${ }^{63}$

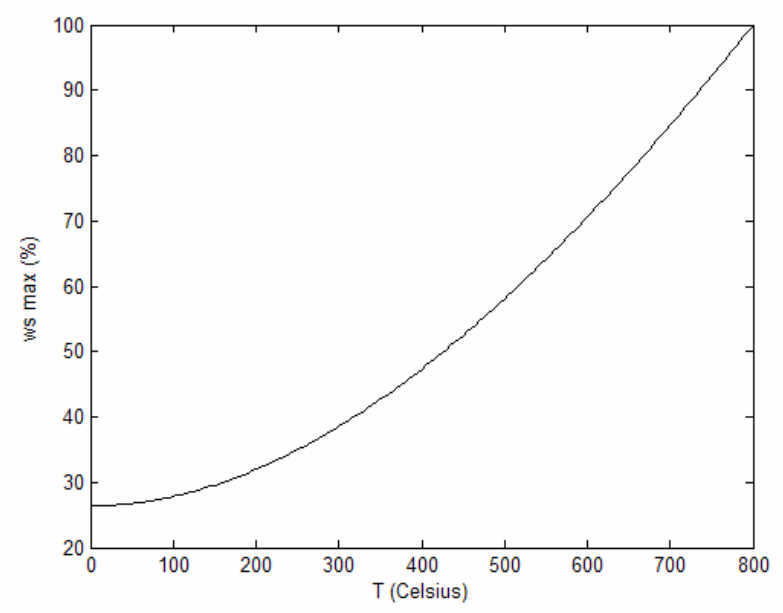

Figure 6. Maximum amount of salt that can be dissolved in liquid (brine) according to Ref. 63

\section{Liquid viscosity}

In general, $\mu_{l}=\mu_{l}\left(P, T, x_{m l}, x_{s l}\right)$. A proposed algorithm, ${ }^{64,56}$ with a few typing errors, compute the liquid viscosity from the pressure, the temperature and the salt weight fraction, i.e., $\mu_{l}=\mu_{l}\left(P, T, \omega_{s}\right)$. This correlation does not consider the methane dissolved in liquid. It assumes $x_{m l}=0$.

\section{Gas molecular diffusion coefficient}

As the gas is a bi-component mixture (methane and water), the molecular diffusion coefficients are equal. 


$$
D_{m g}=D_{w g}=D_{g}
$$

They are assumed equal to the self diffusion coefficient of methane (limit when $y_{w} \rightarrow 0$ ), which was obtained for a given temperature and gas density from a correlation. ${ }^{52}$

\section{Liquid molecular diffusion coefficient}

An algebraic dependence of the molecular diffusion coefficient of methane in a liquid aqueous phase at ambient pressure with the phase viscosity is shown on figure 1 in Ref. 53 by fitting a straight line in that log-log plot

$$
D_{m l}=3.4818 \times 10^{-13} \frac{\mathrm{m}^{2}}{\mathrm{~s}} *\left(\frac{\mu_{l}}{\mathrm{~Pa} \cdot \mathrm{s}}\right)^{-1.2039} .
$$

This relation (obtained from ambient pressure values) considers implicitly the effect of the pressure on the diffusion coefficient through the liquid viscosity. It will be assumed that the molecular diffusion coefficient of the other components in the liquid phase are equal, i.e.,

$$
D_{w l}=D_{s l}=D_{m l}=D_{l}
$$

\subsubsection{Magnitude Orders’ Analysis}

At this point a simple procedure is proposed to evaluate the potential relevance of our formulation in comparison with a Black-oil conventional simulator. A preliminary evaluation of the relative importance of terms involved in the advection-diffusion formalism described in the previous section. In this case we just focus the attention in the water and methane components and salt effect is ignored. Thus, instead of six equations a system of four macroscopic transport equations that model two-phase (gas-water) flow through tight sands is considered for the order's analysis performed here.

From equation (3) and equations (5) and (6) the flow of methane due to convective and dispersive mechanism are given by:

$$
\begin{aligned}
& \vec{V}_{C_{m g}}=M C_{m g} \vec{\nabla} P, \quad \vec{V}_{C_{m l}}=M C_{m l} \vec{\nabla} P, \\
& \vec{V}_{D_{m g}}=M D_{m g} \vec{\nabla} x_{m g} \text { and } \quad \vec{V}_{D_{m l}}=M D_{m l} \vec{\nabla} x_{m l},
\end{aligned}
$$

respectively.

Similarly, but now through equations (3), (8) and (9), the flow of water due to convective and dispersive mechanism will be: 


$$
\begin{array}{ll}
\vec{V}_{C_{w g}}=M C_{w g} \vec{\nabla} P, & \vec{V}_{C_{w l}}=M C_{w l} \vec{\nabla} P \\
\vec{V}_{D_{w g}}=M D_{w g} \vec{\nabla} x_{w g}, & \vec{V}_{D_{w l}}=M D_{w l} \vec{\nabla} x_{w l}
\end{array}
$$

Notice that the contribution of Knudsen diffusion is introduced in the convective term through the parameter $b$ (see equation (34)). Thus, rigorously talking this is not a pure convective term. It is useful for our study to consider the Knudsen diffusion contribution separated from the pure convective term. Thus we express:

$$
\vec{V}_{C_{m g}}=\vec{V}_{P C_{m g}}+\vec{V}_{K n_{m g}} \text {, with } \vec{V}_{P C_{m g}}=\frac{K_{\infty} k r_{g}}{\mu_{g} B_{g}} \vec{\nabla} P \text { and } \vec{V}_{K n_{m g}}=\frac{K_{\infty} k r_{g}}{\mu_{g} B_{g}} \frac{b}{P_{g}} \vec{\nabla} P
$$

Assuming (just for this preliminary estimation) the water-methane moles number's ratio varying only with pressure, in other words, it is assume in this section, that the change in pressure is due to changing the total number of moles in the system by keeping volume, temperature and the proportion of number of moles of water and methane constant. Thus, we can write:.

$$
\vec{\nabla} x_{m g} \approx \frac{\partial x_{m g}}{\partial P} \vec{\nabla} P, \quad \vec{\nabla} x_{m l} \approx \frac{\partial x_{m l}}{\partial P} \vec{\nabla} P
$$

for methane, and

$$
\vec{\nabla} x_{w g} \approx \frac{\partial x_{w g}}{\partial P} \vec{\nabla} P, \quad \vec{\nabla} x_{w l} \approx \frac{\partial x_{w l}}{\partial P} \vec{\nabla} P
$$

for water.

Thus, using equations from (58) to (59) and equations from (63) to (64), and equation (62) we evaluate the ratios:

$$
\begin{aligned}
& \left(\frac{\left.V_{D_{m g}}+V_{D_{m l}}\right)}{\left(V_{C_{m g}}+V_{C_{m l}}\right)}=\frac{M D_{m g} \frac{\partial x_{m g}}{\partial P}+M D_{m l} \frac{\partial x_{m l}}{\partial P}}{M C_{m g}+M C_{m l}},\right. \\
& \frac{\left(V_{D_{w g}}+V_{D_{w l}}\right)}{\left(V_{C_{w g}}+V_{C_{w l}}\right)}=\frac{M D_{w g} \frac{\partial x_{w g}}{\partial P}+M D_{w l} \frac{\partial x_{w l}}{\partial P}}{\left(M C_{w g}+M C_{w l}\right)}, \\
& \frac{\vec{V}_{K n_{m g}}}{\vec{V}_{P C_{m g}}} \approx \frac{\vec{V}_{K n_{w g}}}{\vec{V}_{P C_{w l}}}=\frac{b}{P_{g}}
\end{aligned}
$$


for a representative range of pressures and Klinkenberg permeabilities of tight sands reservoirs.

Rock-fluids phenomena such as the electro-kinetic effect and Knudsen diffusion are captured in our formulations through correlations. The electro-kinetic effects, for the liquid phase, are introduced by considering the klinkenberg permeability elevated by the exponent 1.33 as in the equation (42). The Knudsen diffusion effect is captured from its relation with the Klinkenberg parameter $b$ as explained in the previous section (see equations (37)-(41))

Instead of equation (43)-(46), the relative pemeabilities are simply considered as $k r_{g}=S_{g}$ and $k r_{l}=S_{l}$ here. More realistic curves do not necessarily introduce accuracy in the predictions presented in this section, because the two-phase effective dispersive coefficients are also considered proportional to the phase saturation.

Potential relevance of the dispersive terms introduced in this formulation on the flow predictions can be observed in Figure 7, where maps of the iso-lines of $\left(V_{D_{k g}}+V_{D_{k l}}\right) /\left(V_{C_{k g}}+V_{C_{k l}}\right)$ values, obtained from Equations (65) and (66), and $V_{P C_{m l}} / V_{K n_{m l}}$ by equations (67), in a $\left(K_{\infty}, P\right)$ coordinates' space are shown. Given that pore radius satisfies, approximately, the relation $R \propto \sqrt{K_{\infty}}$ with the permeability, the coordinates' space used here is also representative of a $(R, P)$ Knudsen space.

Figure 7a reveals that the dispersive transport of methane in gas phase can not be neglected in general when the flow is occurring through a porous media of permeability in the range from $10^{-4}$ to $10^{-2} \mathrm{mD}$ for typical temperatures of tight sands reservoirs. Plots show that for a given permeability, when pressure increases from 1 to 500 bar, the dispersive term contribution to flow decreases. In principle dispersive contributions smaller than 0.1 times the "convective” input can be ignored.

Notice in Figure 7, that for temperatures of $240{ }^{\circ} \mathrm{F}$ and $400{ }^{\circ} \mathrm{F}$ the iso-lines are observed only for pressures above 1.72 bar and 17.0 bar, respectively. This is because below these pressures, there is only gas phase and the assumption expressed through Equations (63) and (64) implies that mole fraction gradients are zero and consequently the dispersive contribution vanishes. In the actual reservoirs’ dynamic however, we may have concentration gradients in the gas phase.

In addition, pressure varies with the distance to the well in such a way that it decreases when this distance also decreases and it also varies with production time. Therefore, further evaluation of the dispersive term contribution will be performed in the context of a dynamic reservoir simulation. Section 4.2.2 offers an evaluation in this context. 
From Figure $7 \mathrm{~b}$ it is observed that the dispersive contribution to the flow of water is also relevant in several conditions. In this case for permeabilities larger than $0.01 \mathrm{mD}$ the dispersive contribution to the water flow is not observed significant to the flow for either pressure and temperature value, however we could be underestimating the dispersive input due to relative permeabilities used facilitate the convective flow.

\section{(a)}

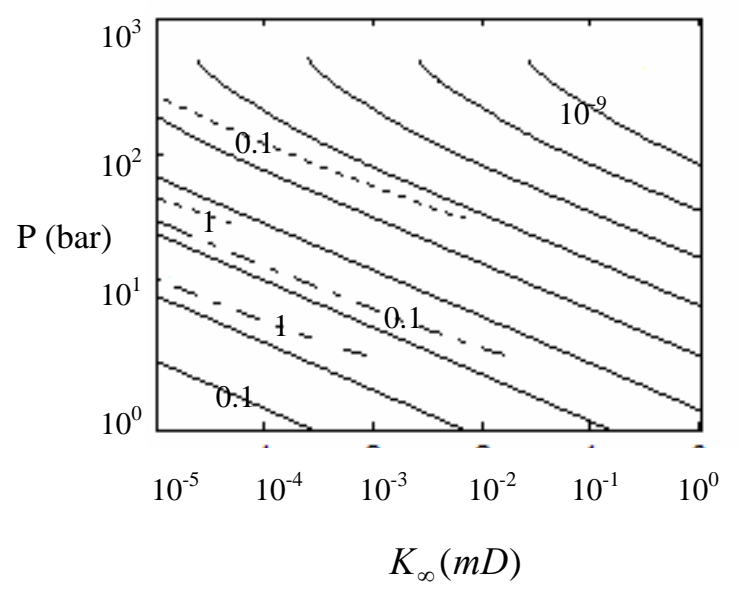

(b)

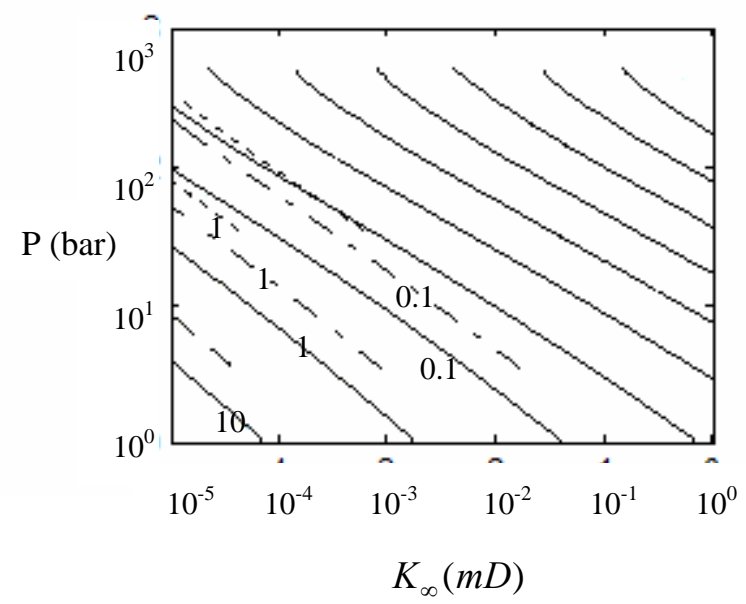

Figure 7. Iso-lines of $\left(V_{D_{k g}}+V_{D_{k l}}\right) /\left(V_{C_{k g}}+V_{C_{k l}}\right)$. (a) Methane $(k=m)$ from Equation (65) and (b) Water $(k=w)$ from Equation (66). Continuous line for $70{ }^{\circ} \mathrm{F}$, dot-dashed line for $240{ }^{\circ} \mathrm{F}$ and dotted line for $400{ }^{\circ} \mathrm{F}$.

Moreover, notice that in equation (50) the dispersion coefficient is being considered for $\mathrm{Pe}<0.3$, which means that we are only considering the diffusive effect. The very known dependence of dispersion coefficient with Pe (Sahimi, 1993), (see Figure 5) would imply that depending on the fluids velocity, the dispersive terms could be up to about four orders of magnitude larger than those estimated in this work. Consequently, when the dependence with Pe is introduced in the estimations we would see that dispersion is actually dominating the fluid flow dynamic through the whole range tight sands permeabilities.

On the other hand, the "convective" term plotted in Figure 7 contains the contribution of the Knudsen diffusion (see equation (62)) through the Klinkenberg parameter $b$. Figure 8 allows to see how much 
of the "convective" contribution is due to the Knudsen diffusion. It is observed that more than $50 \%$ of the "convective" term contribution is dominated by the Knudsen diffusive transport. The increasing direction of Knudsen number $(\mathrm{Kn})$ is also represented in this graph.

We have been considering the Klinkenberg parameter $b$ independent of pressure as conventionally accepted. However, several experimental observations have demonstrated that this parameter is not constant when the mean pore pressure changes. ${ }^{12,13}$ This fact that has been ignored in this preliminary evaluation could also produce an increasing of the relevance of the flow regimes captured in the Klinkenberg parameter $b$. We will go deeper in this matter in the sub-section 4.2 .1 of this report.

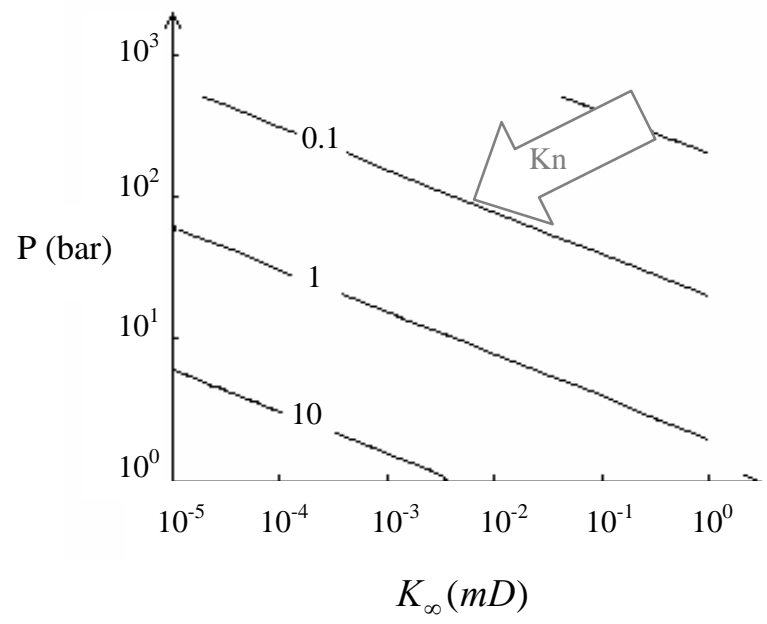

Figure 8. Iso-lines of the ratio $V_{K n_{m l}} / V_{P C_{m l}}=b / P_{g}$ (from equation 9) in a $\left(P, K_{\infty}\right)$ space.

Using Equations (60), (61), and (64) the ratio $\left(V_{D_{w g}}+V_{C_{w g}}\right) /\left(V_{D_{w l}}+V_{C_{w l}}\right)$ allows estimating how much of water flow occurs while the water is in gas phase (vapor). As can be inferred from Figure 9a, most of water flow occurs while the water is in gas phase for typical reservoir conditions.

Figure 9b shows the ratio between dispersive and convective terms presented in the numerator of the ratio plotted in the Figure 3a. According to this figure the dispersive mechanism is non-negligible in general even in this analysis where the dispersive mechanism of flow is under-estimated for the reasons previously discussed. 
(a)

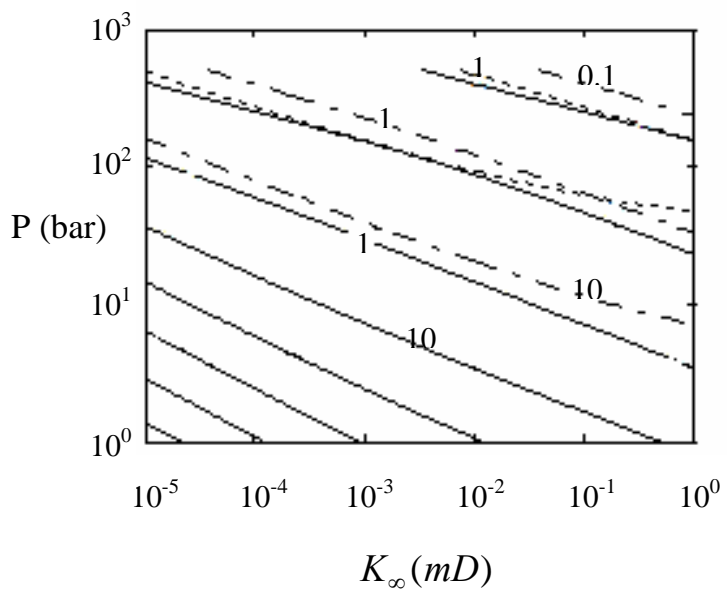

(b)

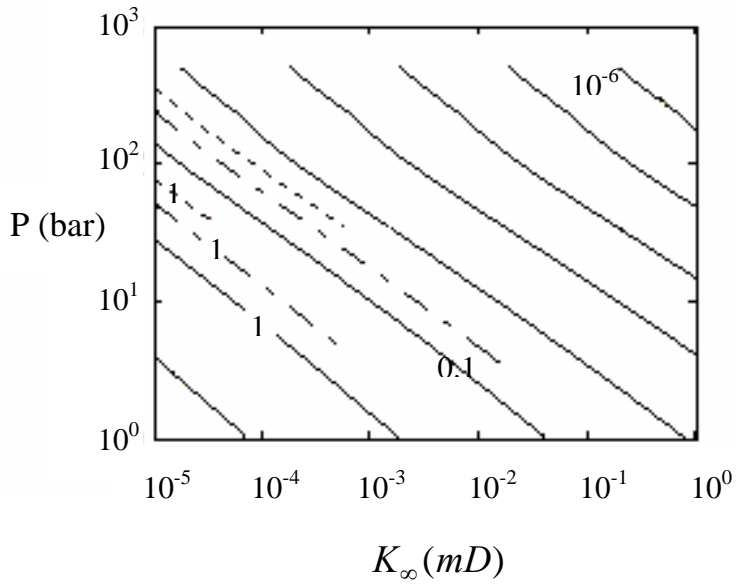

Figure 9. Iso-lines of the ratio (a) $\left(V_{D_{w g}}+V_{C_{w g}}\right) /\left(V_{D_{w l}}+V_{C_{w l}}\right)$ and (b) $V_{D_{w g}} / V_{C_{w g}}$. Continuous line for $70{ }^{\circ} \mathrm{F}$, dot-dashed line for $240{ }^{\circ} \mathrm{F}$ and dotted line for $400{ }^{\circ} \mathrm{F}$.

\subsection{ACTIVITY II: EFFECT OF MICROSCOPIC FLOW MECHANISMS ON GAS-WATER FLOW.}

In previous section it was demonstrated that the impact of Knudsen diffusion on the flow dynamic at macroscopic scale is very important. From that preliminary analysis we conclude that inconsistency on the Knudsen diffusion mechanism of flow could imply a severe lack of prediction not just for the methane but also for water's flow (it was obtained that water flows in gas phase for an extensive range of conditions of tight sands reservoirs).

Sub-section 3.2.1 of this section we will discuss some of the major inconsistencies found in the literature when the Knudsen diffusion are included in the mathematical formalism.

Other aspects that need more detailed analysis are the evaluation of different flow mechanisms in the dynamic context of reservoir simulations. In this sense, some advances were performed and details are offered in the sub-section .2.2.

\subsubsection{Klinkenberg's equation revisited}

One of the most evaluated aspects about gas movement through tight porous structures is the slip flow in the vicinity of pores surfaces. Molecular mean free path in the range from 0.001 to 0.1 times pore size, makes that the slip flow regime goes from relevant to dominant of the flow dynamic behavior; however, from 0.1 to 10 times pore diameter, the transition is the flow regime that most impacts the dynamic. ${ }^{65,66}$ 
In a macroscopic context, based on the Advection Diffusion Model (ADM), the slip flow is frequently captured via Klinkenberg's equation. ${ }^{12}$ Recent researches have not been centered on what for tight rocks reservoirs could be primary. ${ }^{67-69}$ The transition transport regime is not captured in the Klinkenberg deduction and in fact, due to the too-small pore sizes and the mean free path pressure dependence, the transition flow regime could be governing the flow dynamic in these systems. Thus, ADM is not necessarily an adequate alternative to describe the fluid transport when Klinkenberg's equation, as originally proposed, is used to capture those interactions different from moleculemolecule.

In conventional sands, due to the typical pores sizes, the flow is mostly governed by moleculemolecule interaction as represented by Navier-Stoke equation. In contrast, due to the very small pores sizes (from around $5 \mathrm{~nm}$ to $100 \mathrm{~nm}$ ) of unconventional gas reservoirs, contribution of moleculemolecule interactions to the gas transport can be irrelevant and flow regimes associated to moleculewall interactions (slip and transition regime) would play a fundamental role.

Other formalism commonly used is a molecular kinetic based approach known as Dusty Gas Model (DGM). ${ }^{70}$ This formalism has been widely accepted, except for modeling counter-diffusion phenomena. ${ }^{69}$ The established link between the Klinkenberg's equation and the Dusty Gas Model ${ }^{70-}$ ${ }^{76}$ is reasonable considering that both approaches do not include the contribution of the transition flow regime. The relation obtained between the Knudsen diffusivity and the Klinkenberg parameter, $b$, (equation (37)) have given the idea of a more robust understanding of microscopic-macroscopic connection is being obtained. ${ }^{73,76,77}$ This idea is correct considering that DGM introduces the effect of molecule-wall interaction (slip flow) in both the viscous and diffusive term of the advection diffusion equation, but the effect of both, molecule-molecule plus molecule-wall interaction, acting together is neglected, the same as it is in the Klinkenberg's equation. Thus, the Klinkenberg's parameter, $b$, obtained from equation (37) continues being a good approximation only when the molecule-molecule interaction controls the flow at the pore scale. The exclusion of the transition regime in these two famous formalisms possibly explains the absence of explanation for the pressure dependence of the parameter, $b$, experimentally observed..$^{12,13}$

In this sub-section, we will discuss how ADM (with the Klinkenberg's equation included) and DGM are equivalent approaches to describe the gas transport through porous systems and how both models fail when the transition flow regime is playing an important role. We also derived an equation able of describing the so far ignored pressure dependence of the Klinkenberg parameter, $b$. We show experimental evidences of transition flow regime effect on the flow dynamic in porous media and the 
agreement between our model and experimental data. Finally, we will discuss the impacts of the transition flow regime in several porous media under different conditions.

\subsubsection{THEORETICAL APPROACHES}

ADM approach is based on an ad hoc linear addition of the advection term obtained from the Darcy's law and the ordinary diffusive term from the Fick's law, and DGM approach is based on kinetic theory of gases applied to a mixture of gas molecules and large molecules fixed in space which simulate the solid matrix.

In the ADM, the Knudsen diffusion is usually introduced via Klinkenberg's equation, while in the DGM, this flow mechanism is directly derived. A brief discussion of these models, as well as their equivalence and limitations for the prediction of gases transport through porous media will offered in this sub-section..

\section{Advection Diffusion Model (ADM).}

Considering a multi-component gas where:

$$
\eta_{k}=\frac{\text { Number of "type } k^{\prime \prime} \text { Moles }}{\text { Mass of Fluid }} \text { and } \rho=\frac{\text { Mass of Fluid }}{\text { Volume of Fluid }}
$$

the molar concentration of the component $k$, is given by:

$$
c_{k}=\eta_{k} \rho
$$

and for an ideal gas

$$
c_{k}=x_{k} \frac{P_{g}}{R T},
$$

where $x_{k}$ is the molar fraction, $P_{g}$ is the pressure of gas, $T$ the temperature and $R$ the universal gas constant.

The macroscopic averaged concentration is:

$$
C_{k}=\frac{1}{V} \int_{V} c_{k} d V=\phi c_{k}
$$

with $V$ as the representative elemental volume of the porous medium and $\phi$ the porosity.

Thus, for a multi-component gas flowing through an isotropic and homogeneous porous media, the advection-diffusion process of the component $k$, is described by the equation: ${ }^{49}$ 


$$
\frac{\partial}{\partial t}\left(C_{k}\right)+\nabla \cdot\left(C_{k} \vec{u}-\frac{\phi D^{*} P_{g}}{R T} \vec{\nabla} x_{k}\right)=0
$$

where $\vec{u}$ is the mean pore velocity given by:

$$
\vec{u}=\frac{\overrightarrow{\mathrm{v}}}{\phi} \text {, with } \overrightarrow{\mathrm{v}}=-\frac{K_{g}}{\mu_{g}} \vec{\nabla} P
$$

as macroscopic average velocity given by the Darcy equation. As in the previous section, here $K_{g}$ is the porous medium permeability, $\mu_{g}$ is the gas's dynamic viscosity and $\vec{\nabla} P$ is the pressure gradient. Thus, the molar flux of the component $i$, is given by:

$$
\vec{N}_{k}=-\frac{C_{k}}{\phi} \frac{K_{g}}{\mu_{g}} \vec{\nabla} P-\frac{\phi D^{*} P_{g}}{R T} \vec{\nabla} x_{k}
$$

The dispersion coefficient $D^{*}$ was commented in previous section.

Under steady-state and laminar flow condition, Klinkenberg demonstrated that the permeability determined with gases is approximately a linear function of the reciprocal mean pressure, $1 / P_{g}$, in the form expressed by equation (34).

The Klinkenberg permeability, $K_{\infty}$, is the "absolute" permeability to a non polar liquid, usually estimated as the asymptotic value for the limit of $P \rightarrow \infty$. The Klinkenberg parameter, $b$, is a parameter proportional to the mean pressure times the Knudsen Number, Kn, (molecular mean free path divided by a characteristic pore dimension). The traditional way of determining the Klinkenberg parameters is from the plot of permeability vs. reciprocal of mean pressure. The slope of the lineal relation is $k_{\infty} b$ and the intercept is the Klinkenberg permeability, $k_{\infty}$.

This equation have been extensively used, not just in conventional reservoirs rocks, but in low permeability rocks. ${ }^{15,16,20}$ Recent studies about Klinkenberg's equation have been mostly focused on the homogenization of wall-slip gas flow for Knudsen numbers much smaller than one, ${ }^{67}$ the evaluation of robustness of the equation at different scales, assuming validity of it at the local scale, ${ }^{68}$ and the modeling of the Klinkenberg effect under transient gas flow condition. ${ }^{78}$

\section{Dusty Gas Model (DGM). The equivalence with ADM}

The Dusty Gas Model (DGM) as developed by Mason et al, ${ }^{70,79}$ considers the porous material and the gas as a mixture of gases. The solid material (porous media) is represented as large regularly 
distributed particles (dusty molecules) at rest which are treated as one component of the multicomponent ideal gas mixture.

The kinetic theory, through the lowest Chapman-Enskog approximation (first-order deviations from the equilibrium Mawellian velocity distribution), ${ }^{80}$ was applied to the gas mixture, where one of the gas components is the dust molecules. For isothermal conditions the constitutive equations resulting from the DGM are given by (for $k$-component $\neq d u s t$ ):

$$
\sum_{j=1 j \neq k}^{v} \frac{x_{k} N_{j}^{d}-x_{j} N_{k}^{d}}{D_{k j}^{e f f}}-\frac{N_{k}^{d}}{D_{k}^{K}}=\frac{1}{R T} \nabla\left(x_{k} P_{g}\right)
$$

Here $x_{k}\left(x_{j}\right), N_{k}^{d}\left(N_{k}^{d}\right)$ are the mole fraction and the macroscopic total molar diffusive flux of the $k(j)$-component of the gas mixture, respectively. In this formalism, the effective binary diffusivity coefficient $D_{k j}^{e f f}$ is given by: ${ }^{79}$

$$
D_{k j}^{e f f}=(\phi / \tau) D_{k j}
$$

The Knudsen diffusivity, $D_{k}^{K}$, was defined by the relation:

$$
D_{k}^{K}=D_{k}^{d} / x_{d}
$$

and, it can be estimated by: $:^{81}$

$$
D_{k}^{K}=\frac{4}{3} K_{0} \bar{v}_{k}
$$

in which $K_{0}$ is called Knudsen flow parameter, which should be only dependent on the geometry of the pore and the gas-surface scattering law. $\bar{v}_{k}=\sqrt{8 R T / \pi m_{k}}$ is the mean thermal velocity of gas molecules. This relation and equation (78) lead to equation (39) of previous section.

From equation (75), the molar flux $N_{1}^{d}$ for a bi-component gas mixture, is obtained as:

$$
\vec{N}_{1}^{d}=-\frac{x_{1}}{R T}\left(\frac{D_{1}^{K}\left(D_{12}^{e f f}+D_{2}^{K}\right)}{D_{12}^{e f f}+D_{1}^{K} x_{2}+D_{K}^{2} x_{1}}\right) \vec{\nabla} P-\frac{P_{g}}{R T}\left(\frac{D_{1}^{K} D_{12}^{e f f}}{D_{12}^{e f f}+D_{1}^{K} x_{2}+D_{2}^{K} x_{1}}\right) \vec{\nabla} x_{1},
$$

Under an external pressure gradient, viscous fluxes are also contributing to total molar flux of the gas component. To capture their role, the DGM considers it as independent of the diffusive flux term. In fluxes terms, the total molar flux of the $k$-component of the gas is written by: 


$$
\vec{N}_{1}=\vec{N}_{1}^{d}+\vec{N}_{1}^{\mathrm{v}}=\vec{N}_{1}^{d}+x_{1} \vec{N}^{v}
$$

With

$$
\vec{N}^{v}=-\left(\frac{P_{g}}{R T}\right) \frac{K_{\infty}}{\mu_{g}} \vec{\nabla} P
$$

For a single-component gas, equations (75) and (80) imply that the macroscopic molar flux is given by

$$
\vec{N}_{1}=-C_{1} \frac{K_{g}}{\phi \mu_{g}} \frac{\Delta P}{L}, \text { with } K_{g}=K_{\infty}\left(1+\frac{\mu_{g} D_{1}^{K} / K_{\infty}}{P_{g}}\right)
$$

Thus, for the case of a single-component gas flow, the comparison of equation (82) with equation (34) implies:

$$
b=\frac{\mu_{g}}{K_{\infty}} D_{1}^{K}
$$

For a two-component gas, the total molar flux calculated from ADM approach (equations (74) and (34)) is equivalent to those calculated from DGM approaches (equations (79), (80) and (81)) if:

$$
\begin{aligned}
& b=\frac{\mu_{g}}{k_{\infty}}\left(\frac{D_{1}^{K}\left[D_{12}^{e f f}+D_{2}^{K}\right]}{\left(D_{12}^{e f f}+D_{1}^{K} x_{2}+D_{2}^{K} x_{1}\right)}\right) \text { and } \\
& \phi D^{*}=\frac{D_{1}^{K} D_{12}^{e f f}}{\left(D_{12}^{e f f}+D_{1}^{K} x_{2}+D_{2}^{K} x_{1}\right)} .
\end{aligned}
$$

The equation (83) represents the largely accepted link between the Klinkenberg's parameter $b$ and the Knudsen diffusivity $D_{1}^{K}$ already given in equation (37).

Equation (85) signifies that dispersion coefficient of ADM approach, is function of Knudsen and effective molecular diffusivities. In this sense DGM has taken the gas transport equations a step forward by integrating the relevance of the Knudsen diffusion not just in the viscous term but also in the diffusive term.

For a single-component gas, when the pressure gradient is zero ( $P e=0)$, neither DGM nor ADM provide answer on how the effective diffusivity coefficient under both, Knudsen and molecular 
diffusion regimes is. In this case a harmonic mean of the Knudsen and the effective diffusivity was obtained using numerical simulations of gas diffusion in randomly binary media ${ }^{82}$ This result demonstrated to be a good estimation at any Kn value for $P e=0$.

In addition, when the Peclet number is in the region $0.3<P e<10$ and the Knudsen number is in the region $10^{-3}<K n<10$, the transition from continuum to free molecule "flow" is in principle very relevant. ${ }^{65,66}$ However, none of the above discussed approaches is reliable in this probable scenario. The ADM includes the slip flow regime $\left(\sim 10^{-3}<\mathrm{Kn}<10^{-1}\right)$ through the Klinkenberg's equation and the DGM through the set of equations (79) to (81) for a two-component gas and through equation (82) for a single-component gas. However, the transition flow regime $\left(\sim 10^{-1} \leq \mathrm{Kn} \leq 10\right)$ is not captured by none of these approaches. To this point, the transition flow regime seems to be responsible for the pressure dependence of the Klinkenberg's parameter, $b$, experimentally obtained, ${ }^{12,13}$ but not explained.

More sophisticated theoretical approaches based on Kinetic theory could be used to face the lack of reliability presented. Second-orders deviation from the equilibrium Maxwellian velocity distribution, the linearized form of Boltzmann equation and Direct Simulation Monte Carlo (DSMC) ${ }^{66}$ are examples of those, which might quantify with good accuracy the gas flow under transition regime. However, the level of mathematical complexity introduced, the uncertainties in the physical input parameters and the not always consistent physical interpretation of some terms ${ }^{83}$ in the macroscopic equations, become these alternatives practically unattractive, especially if more friendly options are available.

In the next section, an alternative solution to extend the Klinkenberg' equation range of application toward the transition flow regime will be obtained. Our approach offers a solution to this problem in simple and practical way.

\section{Refinement of the Klinkenberg's equation.}

Let us consider the gas flux through a capillary of radio $R$, as illustrated in the Figure 10. The flow is expected to occur under two unlike flow regimes for two distinct regions inside of the capillary, i.e. the region closest to the wall, where molecule-wall interactions are relevant, and the inner region where just molecule-molecule interactions take place. 


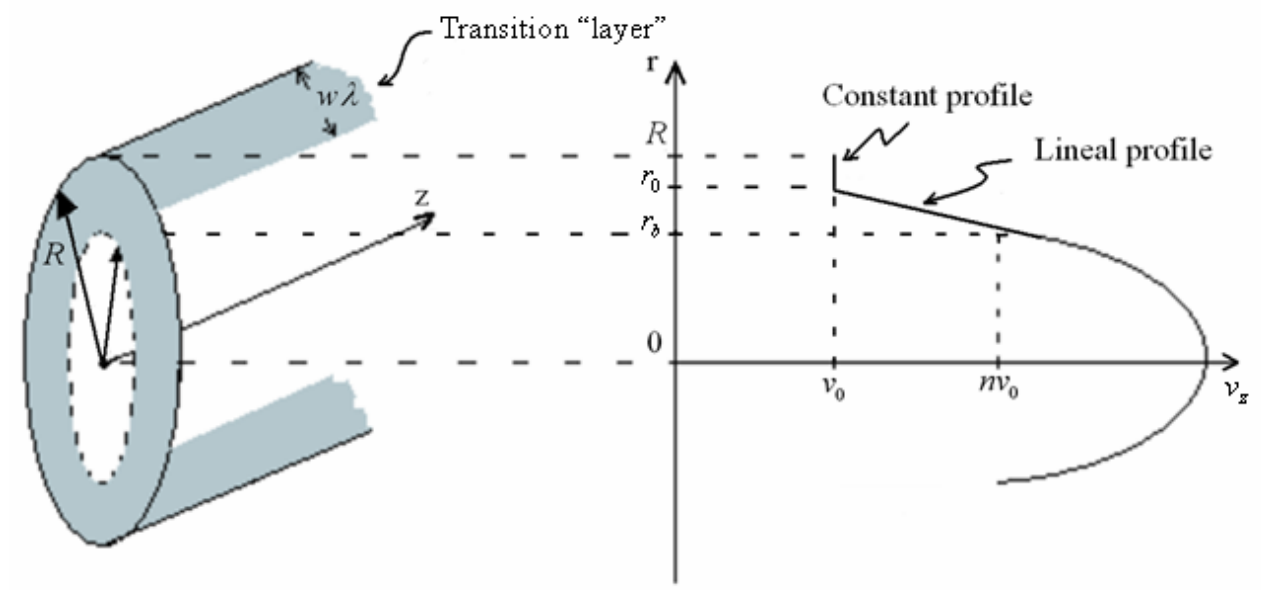

Figure 10. Representation of velocity profiles in a capillary.

For the region between 0 to $(R-w \lambda)$, with $\lambda$ as the mean free path of the gas molecules and $R$ the radii of the capillary, the Navier-Stokes equation are applicable and consequently a Poisseuille velocity profile is appropriate. In the region from $(R-w \lambda)$ to $R$, the interactions molecule-wall plays a role and a velocity profile different from Poisseuille is expected. This region is considered as a transition "layer" where the constitutive laws that defines the stress tensor break down. ${ }^{66}$ For the inner region, the Navier-Stokes equation

$$
\frac{d P}{d z}=\mu_{g}\left[\frac{1}{r} \frac{d}{d r}\left(r \frac{d u_{z}}{d r}\right)\right] .
$$

and under the boundary condition,

$$
u_{z}\left(r_{b}\right)=n v_{0}=v_{b},
$$

the velocity profile is given by:

$$
u_{z}(r)=\frac{1}{4 \mu_{g}}\left[r_{b}^{2}-r^{2}\right]\left(-\frac{d P}{d z}\right)+v_{b} \quad \text { if } 0<r<r_{b} .
$$

In the transition region a lineal profile of velocity is assumed for $r_{b} \leq r \leq r_{0}$ and a constant profile when $r_{0} \leq r \leq R$. This constant profile would occur if a closest to wall layer, where just moleculewall interactions exist. Thus in the transition region the velocity profile would be: 


$$
u_{z}(r)=\frac{v_{0}-v_{b}}{r_{0}-r_{b}}\left(r-r_{b}\right)+v_{b} \quad \text { if } r_{b} \leq r \leq r_{0}
$$

and

$$
u_{z}(r)=v_{0} \quad \text { if } r_{0} \leq r \leq R
$$

where

$$
v_{b}=n v_{0}
$$

and $v_{0}$ is the slip velocity, which is related to the Knudsen Diffusivity coefficient $D^{K}$ by: ${ }^{81}$

$$
v_{0}=\frac{D^{K}}{P_{g}}\left(-\frac{d P}{d z}\right) .
$$

The mean velocity in the capillary is given by:

$$
u=\frac{2}{R^{2}} \int_{0}^{R} u_{z}(r) r d r
$$

In accordance with the Figure 10, we define:

$$
\begin{aligned}
& r_{0}=f_{0} R, \text { with } f_{0}=1-\frac{w_{0} \lambda}{R}, \text { and } \\
& r_{b}=f R, \text { with } f=1-\frac{w \lambda}{R} .
\end{aligned}
$$

Substituting equations from (88) to (90) in equation (93), the capillary mean velocity, is given by:

$$
u= \begin{cases}\frac{R^{2} f^{4}}{8 \mu_{g}}\left(-\frac{d P}{d z}\right)+v_{0}\left(\frac{n-1}{3}\left(f^{2}+f_{0} f+f_{0}^{2}\right)+1\right) & \text { if } f, f_{0}>0 \\ v_{0}\left(\frac{n-1}{3} \frac{f_{0}^{3}}{f_{0}-f}+1\right) & \text { if } f<0, f_{0}>0 \\ v_{0} & \text { if } f, f_{0}<0\end{cases}
$$


The macroscopic mean velocity through a porous media formed by a bundle of capillaries of radius $R$ is given by:

$$
v=\phi u \text {. }
$$

Thus,

$$
v= \begin{cases}\frac{K_{\infty}}{\mu_{g}}\left\{f^{4}+\frac{b_{0 P}}{P}\left(\frac{n-1}{3}\left(f^{2}+f_{0} f+f_{0}^{2}\right)+1\right)\right\}\left(-\frac{d P}{d z}\right) & \text { if } f, f_{0}>0 \\ \mu_{g} \frac{b_{0 P}}{P_{g}}\left(\frac{n-1}{3} \frac{f_{0}^{3}}{f_{0}-f}+1\right)\left(-\frac{d P}{d z}\right) & \text { if } f<0, f_{0}>0 \\ \frac{K_{\infty}}{\mu_{g}} \frac{b_{0 P}}{P_{g}}\left(-\frac{d P}{d z}\right) & \text { if } f, f_{0}<0\end{cases}
$$

Here the Klinkenberg permeability is considered as:

$$
K_{\infty}=\phi \frac{R^{2}}{8}
$$

and $b_{O P}$ is given by previously derived equation (83) as

$$
b_{0 P}=b_{0} \frac{\mu_{g}}{\mu_{g 0}} \text {, with } b_{0}=\frac{\mu_{g 0} D^{K}}{K_{\infty}}
$$

Here we are explicitly considering $\mu_{g}=F(P, T) \cdot \mu_{g 0}$ is the gas viscosity for $P_{g} \rightarrow 0$. A match of equations (98) and (34) implies that Klinkenberg slippage parameter $b$ is given by: 


$$
b= \begin{cases}b_{0 P}\left(\frac{n-1}{3}\left(f^{2}+f_{0} f+f_{0}^{2}\right)+1\right)-\left(1-f^{4}\right) P_{g} & \text { if } f, f_{0}>0 \\ b_{0 P}\left(\frac{n-1}{3} \frac{f_{0}^{3}}{f_{0}-f}+1\right)-P_{g} & \text { if } f<0, f_{0}>0 \\ b_{0 P}-P_{g} & \text { if } f, f_{0}<0\end{cases}
$$

and introducing

$$
p_{0}=\frac{w \lambda P_{g}}{R}
$$

the following non-dimensional quantities are defined

$$
b^{*}=\frac{b}{b_{0 P}}, p^{*}=\frac{P_{g}}{p_{0}}=\frac{1}{1-f} \text {, and } p_{0}^{*}=\frac{p_{0}}{b_{0 P}} .
$$

Thus, the dimensionless slippage parameter is:

$$
b^{*}= \begin{cases}\left(\frac{n-1}{3}\left(f^{2}+f_{0} f+f_{0}^{2}\right)+1\right)-(1+f)\left(1+f^{2}\right) P_{)}^{*} & \text { if } f, f_{0}>0 \\ \left(\frac{n-1}{f_{0}-f}+1\right)-\frac{f_{0}^{3}}{1-f} & \text { if } f<0, f_{0}>0 \\ 1-\frac{P_{0}^{*}}{1-f} & \text { if } f, f_{0}<0\end{cases}
$$

Our model considers two flow regime regions inside the pore (considered as a capillary). The inner region, where Poisseuille parabolic profile is physically valid, and the region adjacent to the wall of pore, where non-Poisseuille flow regime should be expected. The non-Poisseuille region encloses the Knudsen and the transition layers. The velocity profile in the Knudsen region is constant, but the velocity profile in the transition layer, where both, molecule-molecule and molecule-wall, interactions 
take place is unclear. ${ }^{66}$ In order to identify the proper profile, we evaluated a number of profile types for the non-Poisseuille region. These were:

1. Constant Profile in the non-Poisseuille region (Knudsen layer). $\left(r_{0}-r_{b}\right) \rightarrow 0$.

2. Lineal Profile in the non-Poisseuille region (transition layer). $\left(R-r_{0}\right) \rightarrow 0$.

3. Parabolic Profile: The non-Poisseuille region is assumed as a transition layer where the velocity profile is parabolic. Different concavities where studied.

4. Constant-lineal Profile: The non-Poisseuille region is assumed as composed by two layers. One with constant velocity profile (Knudsen) and the other with lineal velocity profile (transition).

Above profile were evaluated under the following considerations:

a) $\quad b_{0 P}=b_{0}$. Ignoring the pressure dependence of the gas viscosity

b) $b_{0 P}=b_{0} \mu_{g} / \mu_{g 0}$. Considering a pressure dependent viscosity.

c) The Knudsen flow equation that captures the geometric effect, given consider Knudsen flow is not taking place in a circular but in an annular capillary. We calculated how the profile would be if the transition region is considered as a Knudsen layer enclosed between two cylinders where the inner cylinder is moving with velocity $\vec{v}$ in the axial direction. The inner cylinder simulates the drag effect that Poisseuille flow could produce over molecules in the transition annular region.

Experimental data reported ${ }^{12,13}$ was used to evaluate the possible theoretical profiles above described.

In Figure 11 we show Klinkenberg parameter $b$ obtained from recent tight sand experiments. ${ }^{13}$ From e-mail communication with the authors of these experiments we verified that these were performed under constant net confining pressure. The absolute permeability to a non-polar liquid was not measured by the authors, but estimated from the curve as the typical procedure, already describe after equation (74) in previous section. Through this method the authors reported $K_{\infty}=0.03 m D$, but the value extrapolated from the data corresponding to higher pressure would be, considering the physical meaning of this parameter, more acceptable. Thus, the value we obtained and used for the assessment illustrated in Figure 11 was $K_{\infty}=0.024 m D$. 


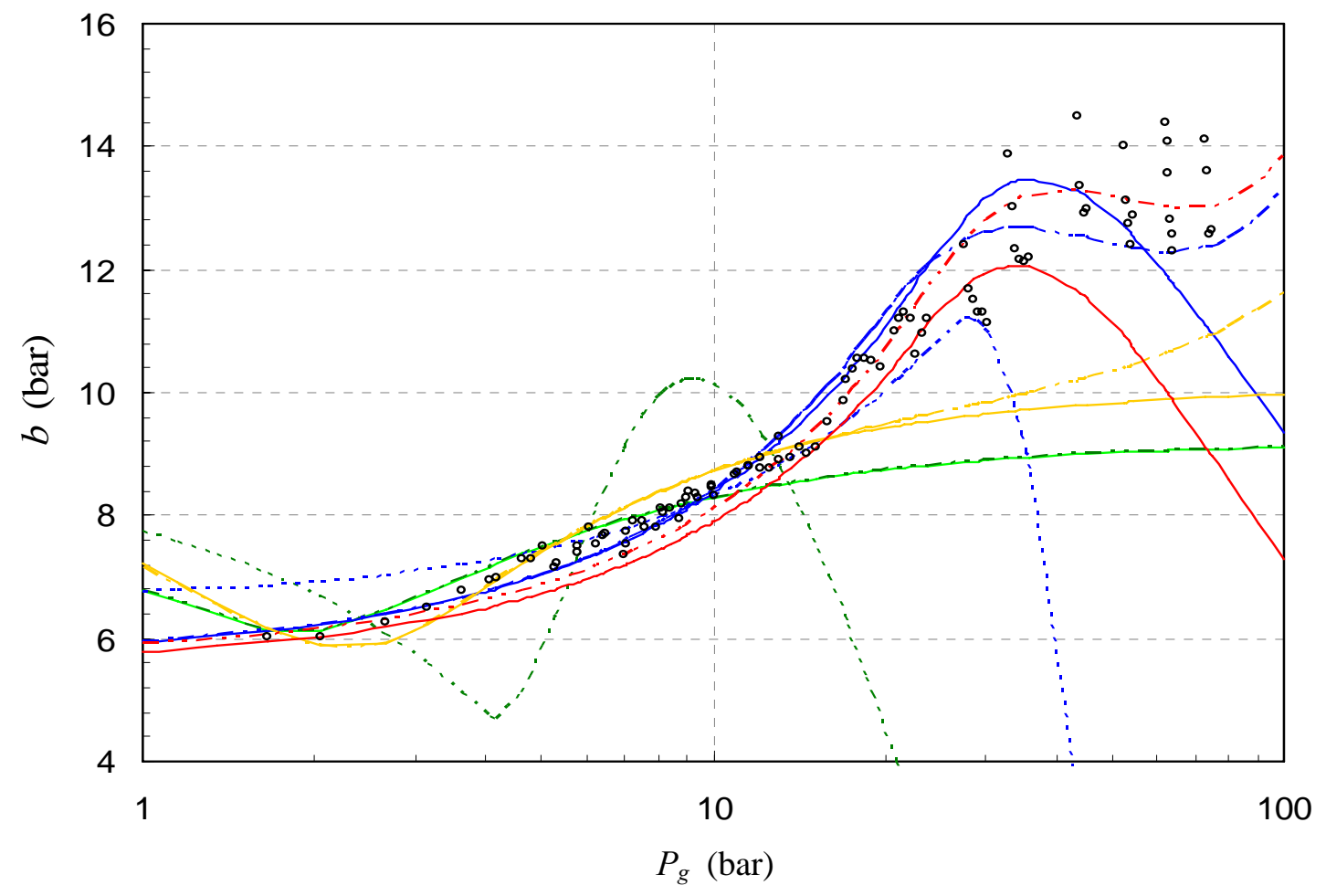

- $\quad$ Experimental, Sample S3, SPE 88472

Constant Velocity Profile $\mu_{g}=\mu_{g 0}$

- - - - Constant Velocity Profile with $\mu_{g}=F(P, T)$

. . . . - . Constant Velocity Profile with variable DK

Linear Velocity Profile $\mu_{g}=\mu_{g 0}$

- - - - Linear Velocity Profile with $\mu_{g}=F(P, T)$

. . . . . - Linear Velocity Profile with variable Dk

Parabolic Velocity Profile $\mu_{g}=\mu_{g 0}$

Parabolic Velocity Profile with $\mu_{g}=F(P, T)$

Constant + Linear Velocity Profile $\mu_{g}=\mu_{g 0}$

- - - - Constant + Linear Velocity Profile with $\mu_{g}=F(P, T)$

Figure 11. Klinkenberg parameter $b$ vs. mean pressure $P_{g}$. Experimental data and theoretical predictions based on different velocity profiles in the non-Poisseuille flow regime region.

Considering that the behavior of parameter $b$ is very sensitive to the value of $K_{\infty}$, values measured with non-polar liquid are highly recommended for future works. 
In all experiments evaluated, linear profile emerges as the best model to reproduce the experimental data. The best match of the constant-lineal velocity profile in the non-Poisseuille region with the data was reached when thickness of the Knudsen region is cero. Others profiles studied were unable of fitting the data under realistic conditions.

An illustration of profiles in the capillary for different Knudsen number is given in Figure 12. This Figure reflects two aspects that deserve a discussion. One is related to the peak-type profile obtained for $\mathrm{Kn}=0.19$ and $\mathrm{Kn}=0.07$. It is logical that considering a lineal profile for the non-Poisseuille region the peak occurs when $w \lambda>R$, however we would expect an smooth curve in $r=0$. Figure 11 evidences that we could not fit the experimental data with a parabolic profile, thus even though deeper analysis should be made in future works, the lineal profile can be accepted a good approximation for practical purposes.

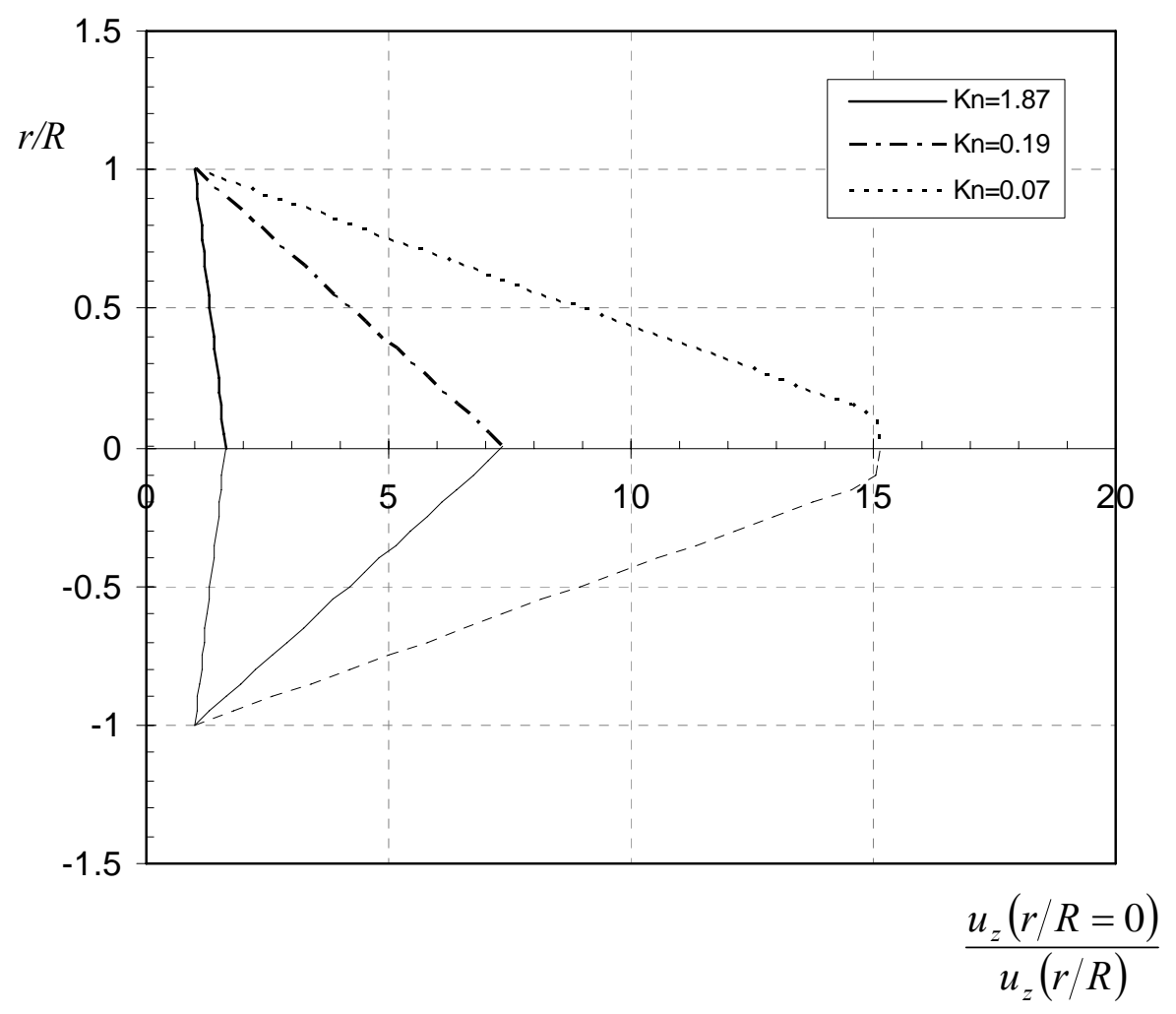

Figure 12. Velocity profiles inside a capillary for different Knudsen Numbers vs velocity ratio, in case of considering linear profile in the non-Poisseuille region. 
The other aspect that requires discussion is that when Knudsen numbers increase our approach predicts a uniform non-dimensional velocity profile. This is an erroneous prediction according to predictions from the linearized Boltzmann solution and Direct Simulation Monte Carlo (DSMC) method. ${ }^{66}$ This discrepancy should be solved in future works; however, our simple model is able of reproducing the experimental data, perhaps because Knudsen numbers are not larger than 5 for our practical situations.

To consider the lineal profile in the non-Poisseuille region in capillaries, we assume $f_{0}=1\left(R=r_{0}\right)$ in equation (129). Figure 13 illustrates curves' family for parameters varying in the reasonable range of values shown in the Table 2.

\begin{tabular}{cl}
\hline \hline Parameter & Values \\
\hline$w$ & 0.1 to 1 \\
$\lambda \mathrm{p}$ & $9.5 \times 10^{-8} \mathrm{~m} \mathrm{~Pa}$ \\
$K_{\infty}$ & $10^{-19}$ to $10^{-13} \mathrm{~m}^{2}$ \\
$R$ & $10^{-9}$ to $10^{-6} \mathrm{~m}$ \\
$\phi$ & 5 to $15 \%$ \\
$D^{K}$ & $10^{-8}$ to $10^{-4} \mathrm{~m}^{2} / \mathrm{s}$ \\
$\mu$ & $1.76 \times 10^{-10}$ bar s \\
\hline \hline
\end{tabular}

Table 2. Parameters involved on the theoretical ranges of the dimensionless quantities.

In all cases $p^{*}<0.1$ implies that equation (100) is a good approximation to determine the Klinkenberg Slippage parameter $b$. Considering that $\mathrm{Kn}=\lambda / R$, the mentioned condition would be equivalent to $\mathrm{Kn}>10$ if $w \approx 1$. According to different regimes of fluid flow taking place under a variety of Knudsen number, ${ }^{65}$ Figure 13 demonstrates that the widely accepted equation (100) is a good approximation close to free molecular flow, but unfortunately the continuum assumption of fluid mechanic starts to be an invalid approximation for these regime. 
(a) $p_{0}^{*}=0.1$

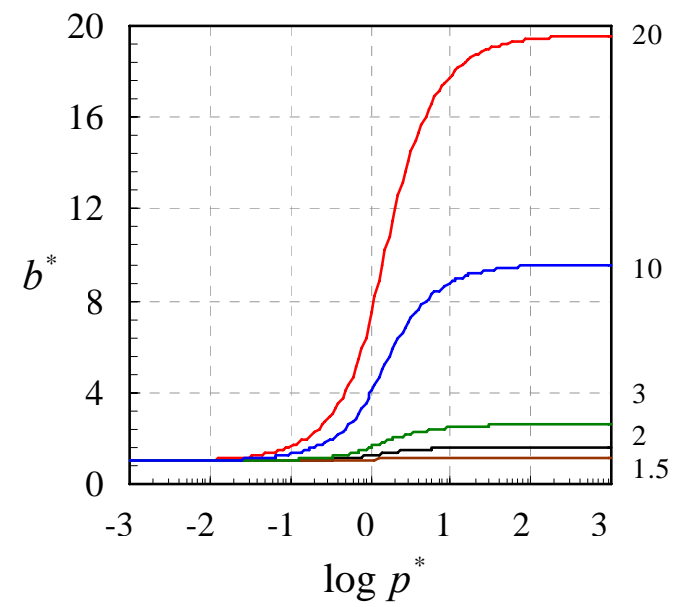

(c) $p_{0}^{*}=1$

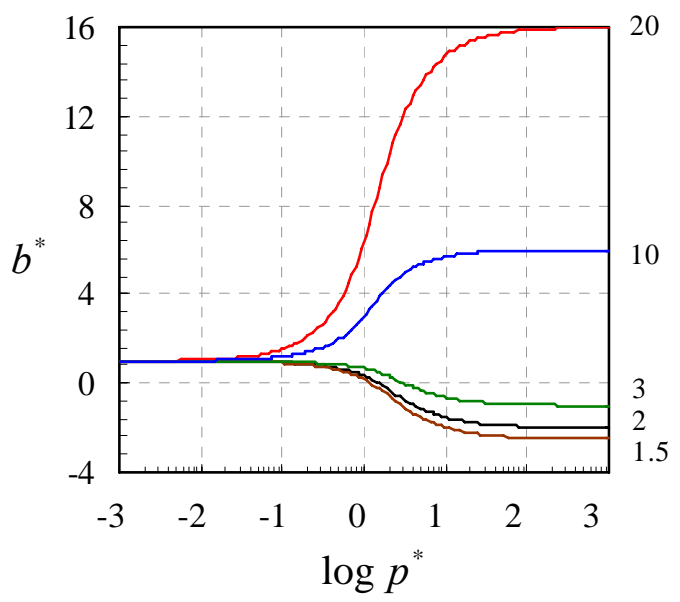

(b) $p_{0}^{*}=0.5$

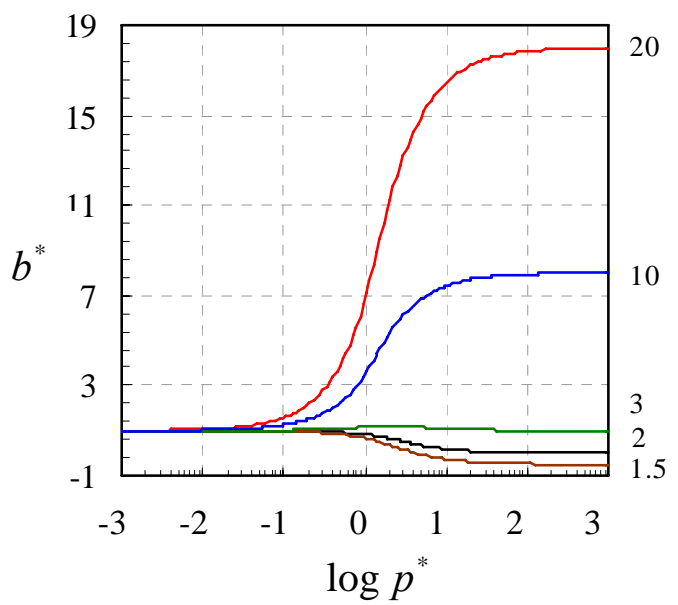

(d) $p_{0}^{*}=5$

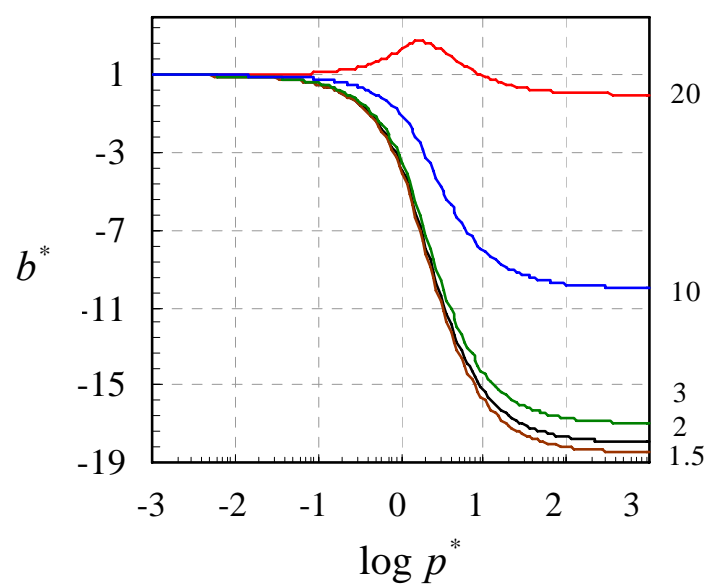

Figure 13. Dimensionless parameter $b^{*}$ considering linear velocity profile in the non-Poisseuille region. Numbers at right represent the values of $n$. Notice that $p^{*}=1 / w \mathrm{Kn}$.

Moreover, Figure 13 (b), (c) and (d) evidence that parameter $b$ could be a negative quantity. This theoretical result, which in principle, looks possible according to the data in Table 2 and Figure 14, is breaking the conventional physical interpretation of the parameter $b$ and Klinkenberg permeability $K_{\infty}$. The equation (34) and the idea of an always-positive parameter $b$, have been the bases to think that permeability measured with gas is always larger than the permeability measured with liquid 
(Traditionally $K_{\infty}$ is assumed equivalent to the permeability to liquid). Figure $\mathbf{1 3}$ is showing a different scenario.

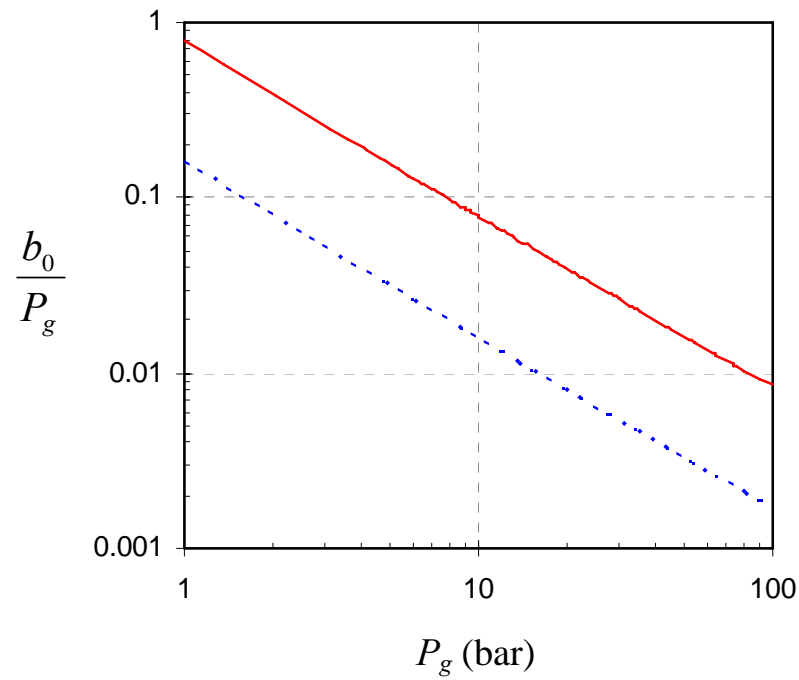

Figure 14. Theoretical evaluation of scenarios c) $p_{0}^{*}=1$ and d) $p_{0}^{*}=5$ in Figure 13. The ordinate, which was obtained from equation (125), is $w \mathrm{Kn}$ for c) and $w \mathrm{Kn} / 5$ for $\mathrm{d}$ ).

In spite of the relevance of this polemic result, further analyses need to be postponed for future works. Experiments designed to revealing the behavior of Klinkenberg parameter $b$, would be essential to clarify this issue. In this sense, we definitely recommend that Klinkenberg permeability be experimentally measured in each experiment, instead of obtained from the extrapolation method. As mentioned before, we verified that behavior of Klinkenberg parameter $b$ is strongly affected by the value of this permeability, which unfortunately was not measured. ${ }^{13}$ Figure 15 shows the sensitivity of the parameter b calculated from this experimental data to the Klinkekenberg permeability $K_{\infty}$. Notice that the Klinkenberg permeability reported by authors $(0.030 \mathrm{mD})$ lead to negative values of $b$. Thus, our controversial theoretical result is an open challenge for experimentalist and theoreticians. 


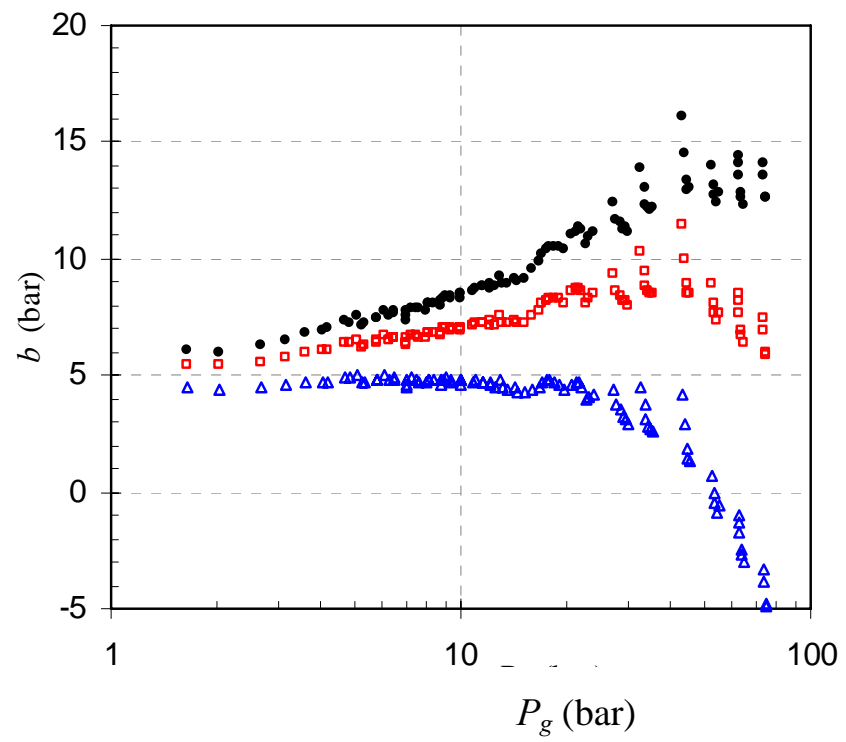

Figure 15. Klinkenberg parameter $b$, obtained from experimental data ${ }^{13}$ considering three different values of Klinkenberg permeability, $K_{\infty}$. Black for $K_{\infty}=0.024 m D$, red for $K_{\infty}=0.026 m D$ and blue for $K_{\infty}=0.030 m D$.

Our efforts for finding experimental data to evaluate the pressure behavior of $b$ and the capability of prediction of our model, just resulted in the data reported in Refs. 12 and 13. Above have been already evaluated the set of data corresponding to tight sands. The other data are the Klinkenberg original experiments, which were not performed for tight sands, but they are useful to evaluate the effect of pressure on the Klinkenberg parameter $b$. Figure 16. shows two Klinkenberg experiments as well as theoretical curves from equation (101) that best match the data. Through this figure we illustrate how the parameter $n$, which is the ratio of velocity in the boundary (in between Poisseuille and non-Poisseuille regions) to the velocity at wall (see equation (87)), is a critical parameter to reach the match between the model and the experimental data. Table 3 shows the match parameters in for Figure 11 (for the lineal profile case) and Figure 16. Equation (78) expresses the dependence of Knudsen diffusivity, $D^{K}$, with pore geometry, the gas-surface scattering law and the mean thermal velocity of gas molecules. The dependence of $n$ with the Knudsen coefficient, through equation (92) and (87), demonstrates that in principle the value of $n$ that best match the three experiments here discussed could be expected to be different as in fact it is. Notice also that the larger $n$, the larger thickness of the non-Poisseuille region $w \lambda$. The values of $w$, resultant of the match parameters are also reported in the table 3. 
(a)

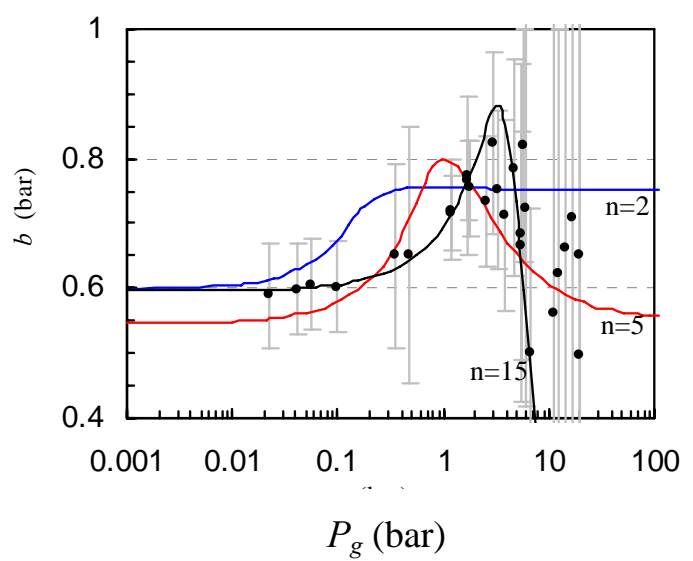

(b)

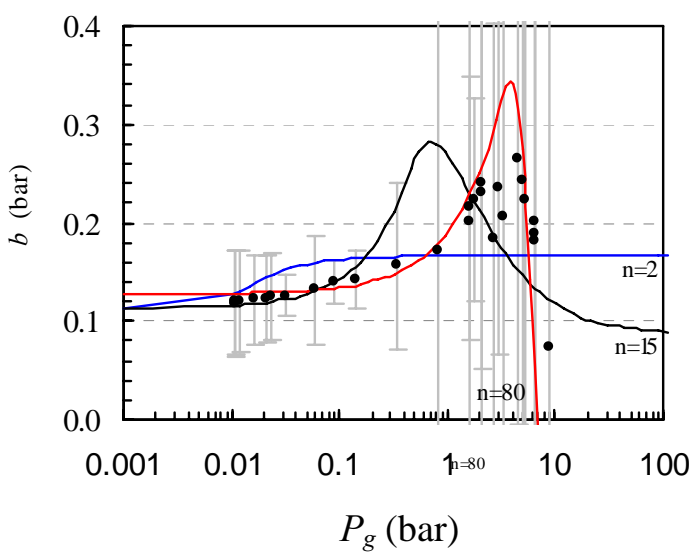

Figure 16. Klinkenberg parameter $b$ vs pressure. (a) $K_{\infty}=2.36 m D$, (b) $K_{\infty}=23 m D$. Dots for experimental data ${ }^{12}$ and lines for theoretical curves from equation (101) for $f_{0}=1$ and parameters shown in table 3 .

If the non-Poisseuille region were only a Knudsen layer the physically plausible value of $n$ would be close to 2, because the reasoning arisen in the original Klinkenberg work. However, the nonPoisseuille region contain also a transition between Knudsen to Poisseuille flow regimes and $n$ is related to the velocity not in the boundary between Knudsen and Transition layers, but in the boundary, where the pure Poisseuille flow profile is taken place.

\begin{tabular}{|c|c|c|c|c|c|c|c|}
\hline \multirow{2}{*}{$\frac{K_{\infty}(\mathrm{mD})}{n}$} & \multicolumn{3}{|c|}{23} & \multicolumn{3}{|c|}{2.36} & \multirow{2}{*}{$\begin{array}{l}\mathbf{0 . 0 2 4} \\
12.8\end{array}$} \\
\hline & 2 & 15 & 80 & 2 & 5 & 15 & \\
\hline$b_{0}$ & 0.110 & 0.112 & 0.128 & 0.596 & 0.546 & 0.596 & 5.69 \\
\hline$P_{0}$ & 0.013 & 0.400 & 3.171 & 0.110 & 0.544 & 2.531 & 17.7 \\
\hline$w$ & 0.17 & 5.14 & 40.7 & 0.155 & 0.766 & 3.560 & 9.48 \\
\hline
\end{tabular}

Table 3. Parameters determined for matching our theoretical approach and experimental data. ${ }^{12,13}$ 
Previous efforts ${ }^{4}$ to clarify the pressure dependence of $b$ are unacceptable because physical inconsistencies involved. The eq. 16 in Ref. 4 shows a misunderstanding of the advection diffusion equation and consequently the pressure dependence of parameter $b$ is explained to be a consequence of considering a real gas. In addition, the physical meaning of the diffusion coefficient $D$ in this formalism was unclear, and arbitrarily assumed as a constant equal to $0.215 \mathrm{~cm}^{2} / \mathrm{s}$. Moreover, gas properties, such as viscosity and gas compressibility, for different pressure and temperature values were calculated from correlations validated for hydrocarbon gases, ${ }^{85,86}$ but not for air which was the gas used in the Klinkenberg experiment that the author proposed to reproduce. Overlooking the misconception of the advection diffusion equation already mentioned, the use of valid correlations for air, ${ }^{87}$ makes that the increasing behavior with pressure of $P \mathrm{c}_{g} \mu$ does not reproduce in any way the increasing behavior with pressure of the parameter $b$.

In general terms we are offering here a model that match to experimental data through a set of parameters that are in the range of physically valid possibilities. The model is revealing that the dependence of $b$ with pressure is due to the transitional flow regime effect on the macroscopic dynamic.

To complement our analysis, we calculate asymptotic and extreme values of equation (101).

The asymptotic values are given by:

$$
\begin{aligned}
& p^{*} \rightarrow 0 \Rightarrow f \rightarrow-\infty \Rightarrow b^{*} \rightarrow 1, \text { and } \\
& p^{*} \rightarrow+\infty \Rightarrow f \rightarrow 1 \Rightarrow b^{*} \rightarrow n-4 p_{0}^{*}
\end{aligned}
$$

When $f \leq 0$, the dimensionless Klinkenberg parameter $b^{*}$, has a linear relation with $p^{*}$. Therefore, extreme values will be investigated just when $f \geq 0$.

The extreme values must satisfy that

$$
0=\frac{d b^{*}}{d f}=\frac{1}{3}(n-1)(1+2 f)-p_{0}^{*}\left(1+2 f+3 f^{2}\right)
$$

A quadratic equation on $f$ is obtained as:

$$
a_{2} f^{2}-a_{1} f-a_{0}=0,
$$

where 


$$
a_{2}=3 p_{0}^{*} \quad a_{1}=\frac{2}{3}(n-1)-2 p_{0}^{*} \quad a_{0}=\frac{1}{3}(n-1)-p_{0}^{*}
$$

Just when $a_{0} \geq 0$ a solution that satisfies $f \geq 0$ is obtained as:

$$
f=\frac{a_{0}+\sqrt{a_{0}\left(a_{0}+a_{2}\right)}}{a_{2}}
$$

Notice that the needed condition $a_{0} \geq 0$ implies that

$$
n-1 \geq 3 p_{0}^{*}
$$

must be satisfied. On the contrary there is not any value of $f$ where the parameter $b^{*}$ makes an extreme value.

The difference between the maximum and the minimum value of $b^{*}$ is calculated as:

$$
\Delta b^{*}=b_{\max }^{*}-b_{\min }^{*}
$$

Where $b_{\max }^{*}$ and $b_{\min }^{*}$ are selected as the maximum and minimum value considering both, the extreme and the asymptotic value. $47 \rightarrow 105$

The definitions given in equations (103) imply that equation (112) is the relative error of $b$, when calculated through the conventional equation (100). Figure 17 shows that this error could be extremely large depending on parameters $p_{0}^{*}$ and $n$. Values of $p_{0}^{*}$ in the range between 0.66 and 2.15 can be obtained for coefficients and petrophysical values corresponding to tight sands gas which contain pore sizes from $1 \mu \mathrm{m}$ into the size dominion of the mean free path of the gas molecules. ${ }^{16,17,20}$ ,19,88 Gray vertical lines enclose the region where curves corresponding to tight sands are expected, and as can be seen the error of $b$ could be larger than a hundred percent. The consequent error in the flow rate of gas would be:

$$
\frac{\Delta v}{v_{0}}=\frac{v_{P}-v_{0}}{v_{0}}=\frac{b^{*}-1}{1+p^{*} p_{0}^{*}}
$$

where $v_{0}$ is the module of the macroscopic velocity of equation (73), with the permeability $K_{g}$ given by equation (34) and the parameter $b$ equal to $b_{0}$ as in equation (100). For $v_{P}$ the equation (101) was used to estimate the parameter $b$. The equations (103) were used to express the error in equation (113) as function of the dimensionless parameters. 


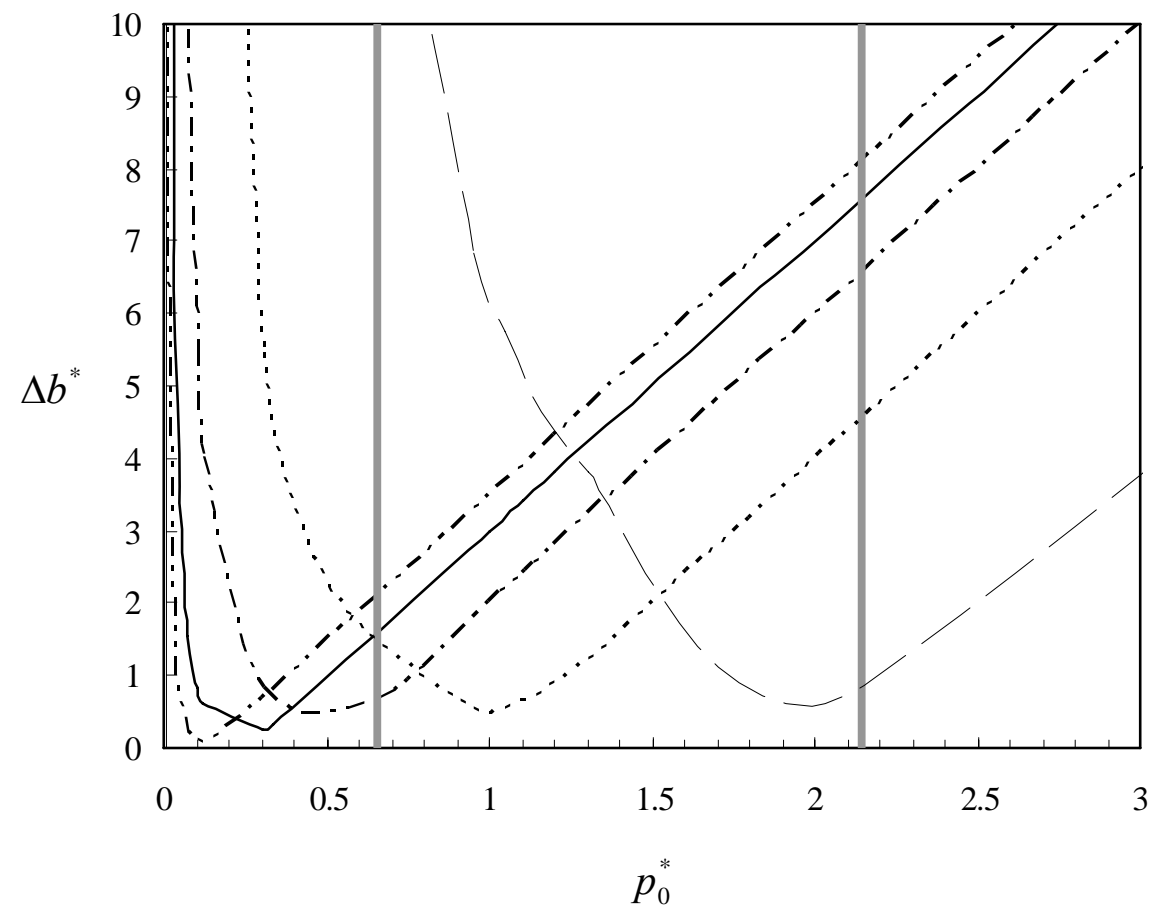

Figure 17. Error of $b^{*}$ (from equation (112)) vs the dimensionless parameter $p_{0}^{*}$ for $n=1.5$ (double dotted dashed line), $n=2$ (solid line), $n=3$ (dotted-dashed line), $n=5$ (dotted line) and $n=10$ (dashed line). Between both gray vertical lines is the region of expected $p_{0}^{*}$ values for tight sand.

Figure 18 shows errors in flow rate prediction vs $p^{*}$, due to ignore the pressure dependence of $b$, for $p_{0}^{*}$ going from 0.6 to 2 and $n$ from 2 to 10 . According to the estimations showed, the discrepancy between the flow rate calculated with the conventional approach and the flow rate calculated through our approach could be huge and consequently the pressure dependence of b should not be ignored in general. Notice that when $\mathrm{Kn}>10^{2}$ (Free molecular flow regime) and $\mathrm{Kn}<10^{-2}$ (Continuum flow regime), the relevance of pressure dependence of $b$ vanishes. In other words, in the slip and transitional flow regime, our model (equation (101)) should be used to estimate the Klinkenberg parameter $b$, however for free molecular and continuum flow regime, the conventional approach to estimate Klinkenberg parameter $b$ is a good approximation. 


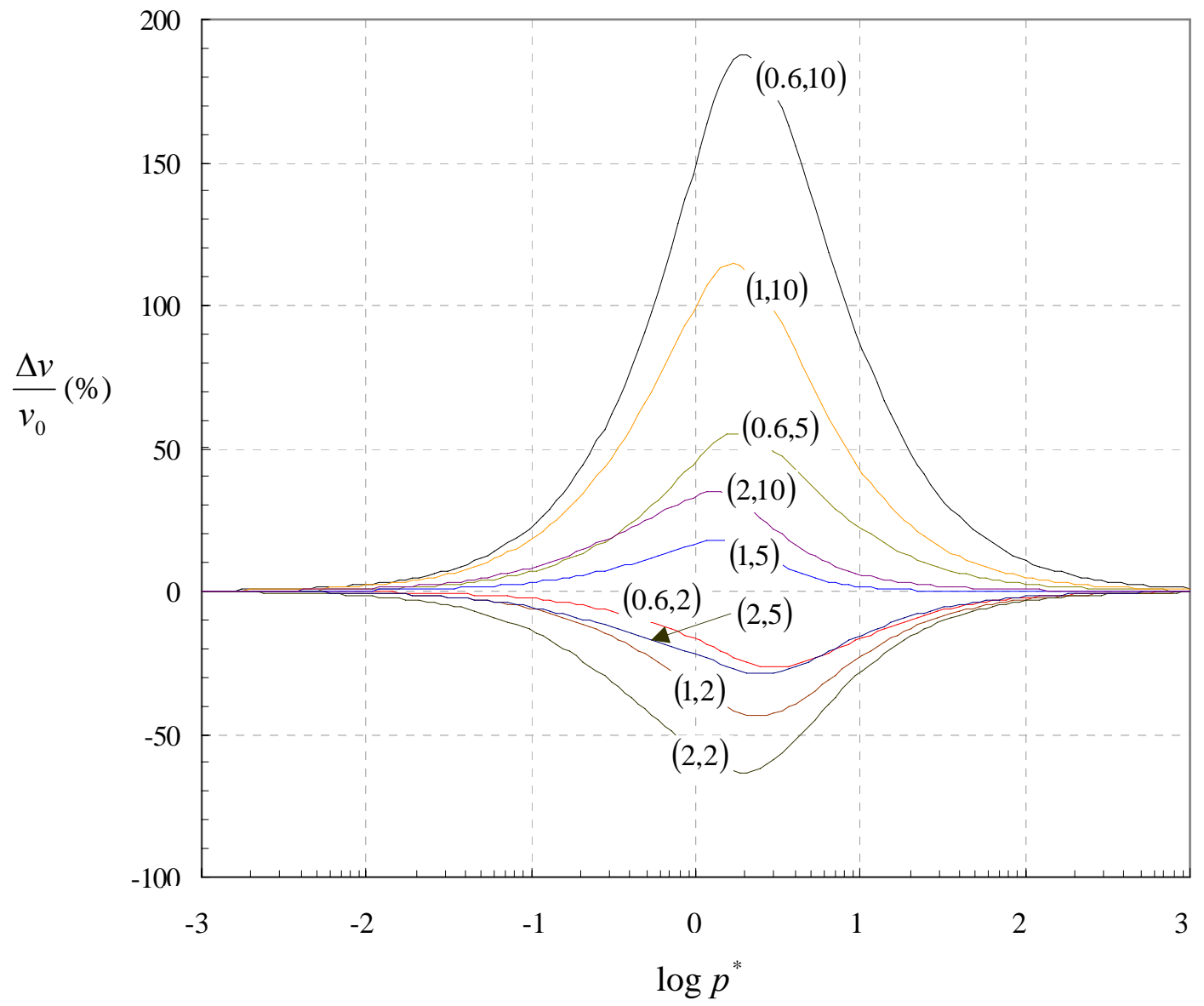

Figure 18. Error in the flow rate of gas (Eq. (55)) vs $p^{*}$. Values $\left(p_{0}^{*}, n\right)$ that originated the curves are shown on them.

\subsubsection{Reservoir Simulator and Sensitivities Studies}

The simulator developed can be characterized as follow:

1. Black-oil in the sense that assumes local phase equilibrium, i.e., components in liquid, gas and solid phase are in equilibrium.

2. Compositional because it tracks all the components.

3. Dual porosity, since consider the matrix system separated from the fracture system and the transfer of mass between them.

4. Multi-mechanistic approach because diffusive mechanism (ordinary and Knudsen (Klinkenberg effect)) and electrokinetic are included. 
5. 1D: assumes radial symmetry

6. Fully implicit: the temporal discretization of the partial differential equations is due to a central differences scheme.

The transport of methane, water and salt in matrix and fractures lead to a system of mass balance equations. The assumption of local phase equilibrium allows to work with the mass conservation equation of every component in all phases, without considering a transfer term among different phases.

The mass conservation equations were describe in the section 4.1 (equations ). These advectiondiffusion equations states that local variation of total mass (in liquid and gas) of methane (equation ) and water (equation ) per unit of total volume is equal to the sum of mass inputs due to convection, diffusion, external sources and transfer between matrix and fracture.

\section{EQUATIONS IN RADIAL SYMMETRY}

Radial symmetry is assumed so that the previous 3D equations (3) and (15) are reduced to 1D equations in the radial coordinate. This is

$$
\frac{\partial F_{k}}{\partial t}=\frac{1}{r} \frac{\partial}{\partial r}\left(r M C_{k g} \frac{\partial P_{g}}{\partial r}+r M C_{k l} \frac{\partial P_{l}}{\partial r}+r M D_{k g} \frac{\partial x_{k g}}{\partial r}+r M D_{k l} \frac{\partial x_{k l}}{\partial r}\right)+\frac{q_{k}}{V_{T}} \mp T_{k}^{M-F} ;
$$

for $k=m, w, s$.

\section{CHANGE TO LOGARITHMIC RADIAL COORDINATES}

In a radial symmetry, the biggest spatial changes occur near the well. In fact, in the single-phase (liquid) case of pure water with constant $B_{l}$ and $\mu_{l}$, the stationary profile from the water balance equation satisfy that

$$
\frac{\partial}{\partial r}\left[r \frac{\partial P}{\partial r}\right]=0 \Rightarrow P=P_{0} \ln \frac{r}{r_{0}}
$$

Another example is the single-phase (ideal gas) case of pure methane with constant $\mu_{g}$ and $B_{g} \propto \frac{1}{P}$. The stationary profile from the methane balance equation satisfy that

$$
\frac{\partial}{\partial r}\left[r P \frac{\partial P}{\partial r}\right]=0 \Rightarrow P^{2}=P_{0}^{2} \ln \frac{r}{r_{0}}
$$


Both examples justify the introduction a new variable

$$
z=\ln \frac{r}{r_{0}} \text {, so that } d z=\frac{d r}{r} \text { and }\left(\frac{\partial A}{\partial r}\right)_{t}=\frac{1}{r}\left(\frac{\partial A}{\partial z}\right)_{t}
$$

In the new variable $z$ the equation (114) transform into

$$
\frac{\partial F_{k}}{\partial t}=\frac{1}{r^{2}} \frac{\partial}{\partial z}\left(M C_{k g} \frac{\partial P_{g}}{\partial z}+M C_{k l} \frac{\partial P_{l}}{\partial z}+M D_{k g} \frac{\partial x_{k g}}{\partial z}+M D_{k l} \frac{\partial x_{k l}}{\partial z}\right)+\frac{q_{k}}{V_{T}} \mp T_{k}^{M-F}=U_{k} ;
$$

for $k=m, w, s$.

\section{NUMERICAL SCHEME}

The region from well radius $\left(r_{1 / 2}\right)$ to the reservoir radius $\left(r_{n_{r}+1 / 2}\right)$ is divided in a number $n_{r}$ of equalspaced intervals in the introduced coordinate $z$. The center of the intervals or cells $\left\{z_{j}\right\}$ in the logarithmic space, which correspond to $\left\{r_{j}\right\}$ in the real space, are related as

$$
z_{j}=\ln \left(\frac{r_{j}}{r_{1 / 2}}\right)=\frac{j-1 / 2}{n_{r}} \ln \left(\frac{r_{n_{r}+1 / 2}}{r_{1 / 2}}\right) \Rightarrow r_{j}=r_{1 / 2} \exp \left(z_{j}\right) ; j=1,2, \ldots, n_{r}
$$

The balance equations (118) are discretized according to a finite difference scheme centered in space. Thus, the mass flux at the upper face of the cell $j$, is obtained as

$$
\left(M \frac{\partial X}{\partial z}\right)_{j+1 / 2}=M_{j+1 / 2}^{\text {upstream }} \frac{\left(X_{j+1}-X_{j}\right)}{\Delta z} ; j=1,2, \ldots, n_{r} .
$$

Here $M$ represent the mobility and $X$ the driven parameter at cells (pressure for convective flow or molar fraction for diffusive flow). The upstream weighting of the mobility between cells gives better numerical results than an averaged mobility. ${ }^{84}$ Considering that the flux goes from higher to lower $X$, the upstream mobility is

$$
M_{j+1 / 2}^{\text {upstream }}=\left\{\begin{array}{cc}
M_{j} & X_{j}>X_{j+1} \\
M_{j+1} & X_{j}<X_{j+1}
\end{array} ; j=1,2, \ldots, n_{r} .\right.
$$

Then an accumulation rate term at cell $j$ is computed as 


$$
\left[\frac{1}{r^{2}} \frac{\partial}{\partial z}\left(M \frac{\partial X}{\partial z}\right)\right]_{j}=\frac{1}{r_{j}^{2} \Delta z}\left[\left(M \frac{\partial X}{\partial z}\right)_{j+1 / 2}-\left(M \frac{\partial X}{\partial z}\right)_{j-1 / 2}\right] ; j=1,2, \ldots, n_{r} .
$$

The numerical scheme was also centered in time (implicit). Representing the system of balance equations (118) as

$$
\frac{\partial \vec{F}}{\partial t}=\vec{U}
$$

the discretized version in the time interval $\Delta t=t^{n+1}-t^{n}$ is

$$
\frac{\vec{F}^{n+1}-\vec{F}^{n}}{\Delta t}=\frac{\vec{U}^{n+1}+\vec{U}^{n}}{2}
$$

The system of balance equations (124) has $6 n_{r}$ equations, which contain the $6 n_{r}$ molar densities at time $t^{n}\left(\vec{n}^{n}\right)$ and the ones at time $t^{n+1}\left(\vec{n}^{n+1}\right)$.

\section{NUMERICAL PROCEDURE}

The numerical algorithm can be summarized in the following steps:

1) A uniform distribution of the number of moles per unit volume (or molar density) of the three components for matrix and fractures is assigned as initial condition $\left(\vec{n}^{0}\right)$. The molar densities $\left(n_{m}, n_{w}, n_{s}\right)$ are obtained from the initial pressure $\left(P=P_{g}\right)$ and gas saturation $\left(S_{g}\right)$ in the reservoir, at the given temperature $(T)$.

2) The phase equilibrium is obtained from the molar density of the three components $\left(n_{m}, n_{w}, n_{s}\right)$ at the given temperature $(T)$, and the related parameters are determined in each site for matrix and fractures. They are pressure $\left(P=P_{g}\right)$, gas phase saturation $\left(S_{g}\right)$ and phase composition ( $x_{w g}, x_{m l}, x_{s l}$ and $n_{s s}$ ). From them, other necessary parameters are also determined ( $B_{g}, B_{l}, R_{s g}, R_{s l}, \omega_{s}, \rho_{l}^{s c}, D_{g}, D_{l}, \mu_{g}, \mu_{l}, K_{g}, K_{l}, k r_{g}$, and $k r_{l}$ ).

3) The system of balance equations (124) is solved simultaneously for the molar densities in the next time step ( $\vec{n}^{n+1}$ ) from the previous molar densities $\left(\vec{n}^{n}\right)$ and by applying the boundary conditions.

4) With new spatial distribution of molar densities $\left(\vec{n}^{n+1}\right)$, it is returned to step 2), if the abandon criterion is not reached. 


\section{SOLUTION OF THE SYSTEM EQUATIONS}

The unknown molar densities $\left(\vec{n}^{n+1}\right)$ are determined from the nonlinear system of equations (124), by using a Newton-Rapson iterative method. Let $\vec{n}_{*}^{n+1}$ be the exact solution of the system (124), so that

$$
\frac{\vec{F}_{*}^{n+1}-\vec{F}^{n}}{\Delta t}=\frac{\vec{U}_{*}^{n+1}+\vec{U}^{n}}{2}
$$

If $\vec{F}^{n+1}$ and $\vec{U}^{n+1}$ are obtained from an approximate solution $\vec{n}^{n+1}$, which is close to the real solution $\vec{n}_{*}^{n+1}$, then a Taylor series expansion to the first order gives

$$
\vec{F}_{*}^{n+1}=\vec{F}^{n+1}+\sum_{j} \frac{\partial \vec{F}^{n+1}}{\partial\left(\vec{n}^{n+1}\right)_{j}}\left(\vec{n}_{*}^{n+1}-\vec{n}^{n+1}\right)_{j} ; \vec{U}_{*}^{n+1}=\vec{U}^{n+1}+\sum_{j} \frac{\partial \vec{U}^{n+1}}{\partial\left(\vec{n}^{n+1}\right)_{j}}\left(\vec{n}_{*}^{n+1}-\vec{n}^{n+1}\right)_{j} .
$$

Substituting those expansions in (125), follows

$$
\vec{A} \cdot\left(\vec{n}_{*}^{n+1}-\vec{n}^{n+1}\right)=\vec{B}^{n+1}+\vec{B}^{n}
$$

where

$$
\vec{B}^{n+1}=\frac{\Delta t}{2} \vec{U}^{n+1}-\vec{F}^{n+1} ; \vec{B}^{n}=\frac{\Delta t}{2} \vec{U}^{n}+\vec{F}^{n} ;(\vec{A})_{i, j}=-\frac{\partial\left(\vec{B}^{n+1}\right)_{i}}{\partial\left(\vec{n}^{n+1}\right)_{j}} .
$$

The system of equations to solve (127) for $\Delta \vec{n}^{n+1}=\vec{n}_{*}^{n+1}-\vec{n}^{n+1}$ is highly sparse, because the matrix $\vec{A}$ has no more than 12 non-zero elements per row. After testing the available methods to solve this system in MatLab, the Bi-conjugate Gradients Stabilized Method (function bicgstab) with preconditioned matrices obtained from LU factorization was chosen because it was the fastest method.

Thus, the iterative procedure start from determining the array $\vec{B}^{n}$ given $\vec{n}^{n}$ and the array $\vec{B}^{n+1}$ given the approximate solution $\vec{n}^{n+1}$, which at the first iteration is just equal to $\vec{n}^{n}$. Small deviations are added to the elements of $\vec{n}^{n+1}$ and the corresponding values of $\vec{B}^{n+1}$ are obtained so that the derivatives in $\vec{A}$ (128) can be found. Then the system of equations (127) are solved and a better approximation for $\vec{n}^{n+1}$ is achieved. The iteration continues (usually 3 to 6 times) until the maximum absolute value of $\Delta \vec{n}^{n+1}$ is small enough.

\section{VARIABLE TIME STEP METHOD}


The variable time step method tries to reach solutions with acceptable errors in a minimum computing time.

Once the new solution $\vec{n}^{n+1}$ is obtained from the Newton-Rapson method, the convergence criterion is tested, which is that the maximum relative variation in $n_{m}$ and $n_{w}$ does not exceed a given amount. After several tests the maximum relative variation allowed was set to $2 \%$. If the convergence criterion is not satisfied, then the solution is rejected and the time step is reduced to find a new solution. The reduced time step is computed trying that the maximum relative variation would be around the half of the allowed value (1\%). If the solution meets the convergence criterion, it is accepted and the next time step is guessed from one fourth of maximum relative variation allowed in $n_{m}$ and $n_{w}(0.5 \%)$. The value chosen for next time step can not be more than two times the previous time step, though. This way of selecting of the time step prevents that most of the time the convergence criterion is satisfied for the next solution.

\section{BOUNDARY CONDITIONS}

Boundary conditions need to be specified at the outer boundary and at the inner boundary (well). This model considers that there is no flow through the face boundaries, which means following the notation of equation (122) that

$$
\left(M \frac{\partial X}{\partial z}\right)_{1 / 2}=\left(M \frac{\partial X}{\partial z}\right)_{n_{r}+1 / 2}=0 .
$$

The boundary condition is set by considering external source terms $q_{k} / V_{T}$ in equations (118) at the two boundary cells, though. At the outer boundary there are two options as boundary conditions:

1. No flow condition. This means that $q_{k_{n_{r}}}^{j}=0$ for $k=m, w, s$ and $j=M, F$, which corresponds to an isolated reservoir.

2. Constant molar density condition. Here, $n_{k_{n_{r}}}^{j}=$ constant and $\frac{\partial F_{k n_{r}}^{j}}{\partial t}=U_{k n_{r}}^{j}=0$ for $k=m, w, s$ and $j=M, F$. This option corresponds to an unlimited and instantaneous supply from outside.

At the first cell the boundary condition has also two options:

1. Constant mass flow rate of methane $\left(q_{m 1}=q_{m 1}^{M}+q_{m 1}^{F}\right)$.

2. Constant well pressure $\left(P^{\text {well }}\right)$. 
At the well the mass flow rate of the three components in matrix and fractures are assumed proportional to the convective mobility at the first cell, so that

$$
\frac{q_{k 1}^{j}}{V_{T 1}}=2 \pi\left(r_{1}+\frac{\Delta r_{1}}{4}\right) h \cdot M C_{k 1}^{j}\left(\frac{P^{\text {well }}-P_{g_{1}}^{j}}{\Delta r_{1} / 2}\right) \text { for } k=m, w, s \text { and } j=M, F \text {. }
$$

Equation (130) is used to determine the six flow rates from the well pressure, necessary for equations (118) at the first cell. In the second option, the well pressure is given directly, but in the first one, the well pressure is determined from the mass flow rate of methane as

$$
P^{\text {well }}=\frac{q_{m 1}+M^{M} P_{g}^{M}+M^{F} P_{g}^{F}}{M^{M}+M^{F}} ; \text { where } M^{j}=\frac{2 V_{T 1}}{\Delta r_{1}} M C_{m 1}^{j} .
$$

\section{MASS FLUXES}

In further sections the convective and diffusive transport are compared. In this sense, relations similar to (120) are used so that the convective and diffusive mass flow rates through the cell boundaries are respectively,

$$
\begin{aligned}
& V C_{k i}=2 \pi h \cdot M C_{k i} \frac{\partial P_{i}}{\partial z}, i=g, l ; \text { and } \\
& V D_{k l}=2 \pi h \cdot M D_{k l} \frac{\partial x_{k}}{\partial z}, V D_{k g}=2 \pi h \cdot M D_{k g} \frac{\partial x_{k g}}{\partial z} ; \text { for } k=m, w, s .
\end{aligned}
$$

From this definition the equation (118) becomes

$$
\frac{\partial F_{k}}{\partial t}=\frac{1}{2 \pi h r^{2}} \frac{\partial}{\partial z}\left(V C_{k g}+V C_{k l}+V D_{k g}+V D_{k l}\right)+\frac{q_{k}}{V_{T}} \mp T_{k}^{M-F}=U_{k} .
$$

From the definition of the accumulation terms (4), (7) and (10) and using that in radial coordinates

$$
V_{T}=\pi\left(\left(r+\frac{\Delta r}{2}\right)^{2}-\left(r-\frac{\Delta r}{2}\right)^{2}\right) h=2 \pi h r \Delta r
$$

plus the relation (117), equation (133) transform into

$$
\frac{1}{2 \pi h r \Delta r} \frac{\partial m_{k}}{\partial t}=\frac{1}{2 \pi h r} \frac{\partial}{\partial r}\left(V C_{k g}+V C_{k l}+V D_{k g}+V D_{k l}\right)+\frac{q_{k}}{V_{T}} \mp T_{k}^{M-F}=U_{k} .
$$


From (135) it is understood why the factor $2 \pi$ must be introduced on definitions (132) in order to obtain the mass flow rates through the radial boundaries.

\section{SENSITIVITIES}

Sensitivities of the multi-mechanistic formulation, detailed in previous sections, to some reservoir parameter were performed. Parameters used for the base case are shown from Table 4 to Table 7. Most of parameters correspond to the upper cozzette blanket sand. ${ }^{28,89}$

\begin{tabular}{|c|c|c|c|}
\hline \multicolumn{4}{|c|}{ Reservoir Geometry } \\
\hline$r_{w}(\mathrm{in})$ & $r_{T}(\mathrm{ft})$ & $L z(\mathrm{ft})$ & $n_{\mathrm{r}}$ \\
\hline 3.5 & 1500 & 30 & 50 \\
\hline
\end{tabular}

Table 4. Geometric parameter used as base case.

Reservoir and flow parameters

\begin{tabular}{|c|c|c|c|c|c|c|c|}
\hline$\phi^{M}=\phi^{F}$ & $K_{\infty}(\mathrm{mD})$ & $S_{g i}$ & $S_{l i}$ & $P_{d}$ (bar) & $b$ (bar) & $l(\mathrm{~m})$ & $k r_{i}, P c$ \\
\hline $0.0345^{\mathrm{a}}$ & $0.001^{\mathrm{b}}$ & $0^{\mathrm{b}}$ & $0.25^{b}$ & $20^{\mathrm{b}}$ & $0^{\mathrm{b}}$ & 7 & Corey $\xi=2.4^{\mathrm{b}}$ \\
\hline
\end{tabular}

Table 5. Reservoir and flow parameters used as base case. ${ }^{\mathrm{a}}$ Total porosity equal to $0.069 .{ }^{\mathrm{b}}$ considered equal for matrix and fractured space

\begin{tabular}{|c|c|c|c|c|}
\hline \multicolumn{5}{|c|}{ Initial and boundary conditions } \\
\hline$S_{g}$ & $P_{g r}$ (bar) & $T_{r}\left({ }^{0} \mathrm{~F}\right)$ & $\omega_{s}$ & boundary \\
\hline 0.60 & 434 & 230 & $10^{-4}$ & closed $^{\mathrm{a}}$ \\
\hline
\end{tabular}

Table 6. Initial and boundary conditions for base case. ${ }^{a}$ No flow through the boundary allowed. 


\begin{tabular}{ccc}
\hline \hline \multicolumn{3}{c}{ Well production history } \\
\hline$\frac{t_{p} \text { (days) }}{365}$ & $\frac{P_{i w} \text { (bar) }}{1}$ & Well \\
& $\frac{\text { Production }}{365}$
\end{tabular}

Table 7. Data related to well production history used for the base case

In the previous section we showed the theoretical estimation of possible errors due to consider the parameter $b$ as independent on pressure. Those calculations were performed for air, as in the experiments involved. However, methane is the gas of our concern and important difference in pressure dependence of parameters exist. In Figure 19 we show how the Klinkenberg parameter $b$ change by consider air or methane as the gas and also if the temperature is the standard of the reservoir temperature. $D^{K} / K_{\infty}=4.41 \times 10^{10} \mathrm{~s}^{-1}$ resulted from the match with experimental data of Ref. 13 while $D^{K} / K_{\infty}=2.84 \times 10^{10} s^{-1}$ resulted from our correlation (equation (38)) for the base case permeability (See Table 5). Thus, the black curve corresponds to our base case. Notice that the black curve shows the largest pressure dependence ever since low pressures. Thus, our previous estimations (performed to air) are underestimating the pressure effect on the parameter $b$.

Figure 20 shows the Klinkenberg parameter $b$, for the range of permeabilities used in the simulations. For pressure larger than 300 bar, larger slopes of curves are obtained. Also notice that negative values of the parameter $b$ are obtained for permeabilities larger than $10^{-2} \mathrm{mD}$. In previous section, where our model for $b$ was presented, we discuss this controversial result. Here, we emphasize that negatives values of b imply that the permeability to the gas could be smaller than permeability to a non-polar liquid. In principle, for certain range of Knudsen numbers this effect could be explained; however, further experimental and theoretical studies are recommended to validate or refute this behavior. Meanwhile, our model expressed through the equation (101) is used in the simulations here presented. 
(a)

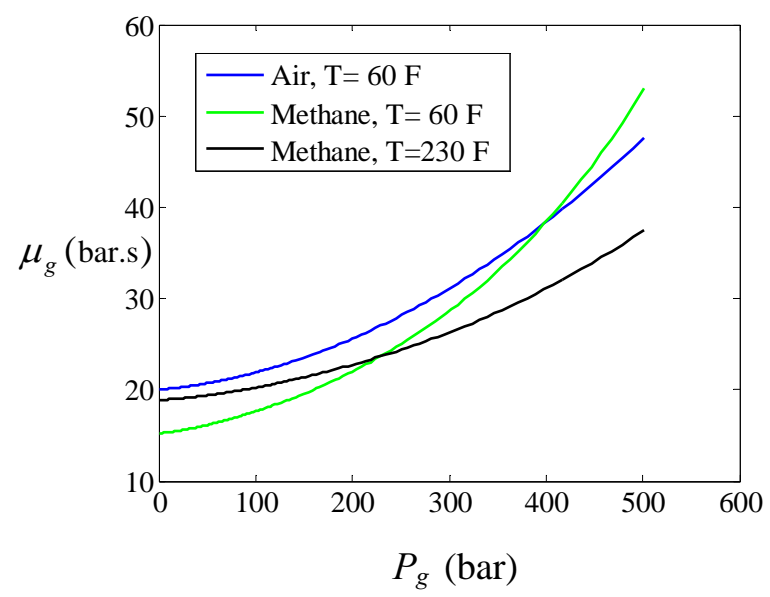

(b)

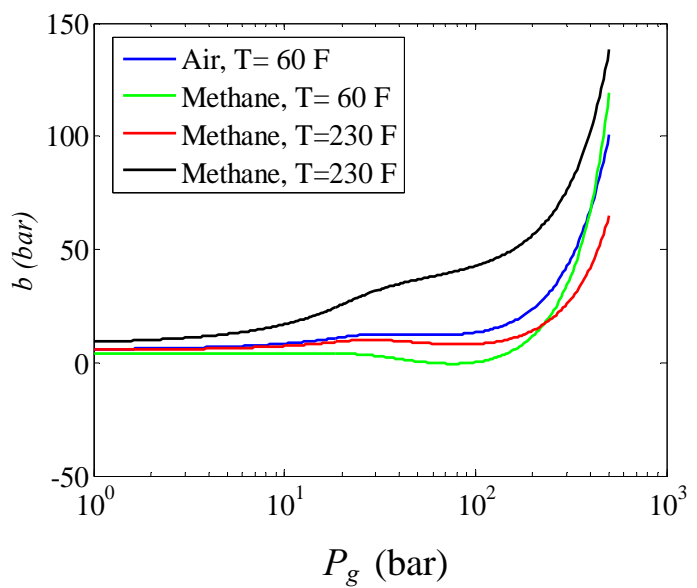

Figure 19. Effect of consider air or methane under different temperatures. (a) Gas viscosity, (b) Klinkenberg parameter $b$ with $D^{K} / K_{\infty}=4.41 \times 10^{10} s^{-1}$ for black and $D^{K} / K_{\infty}=2.84 \times 10^{10} s^{-1}$ for the others curves.

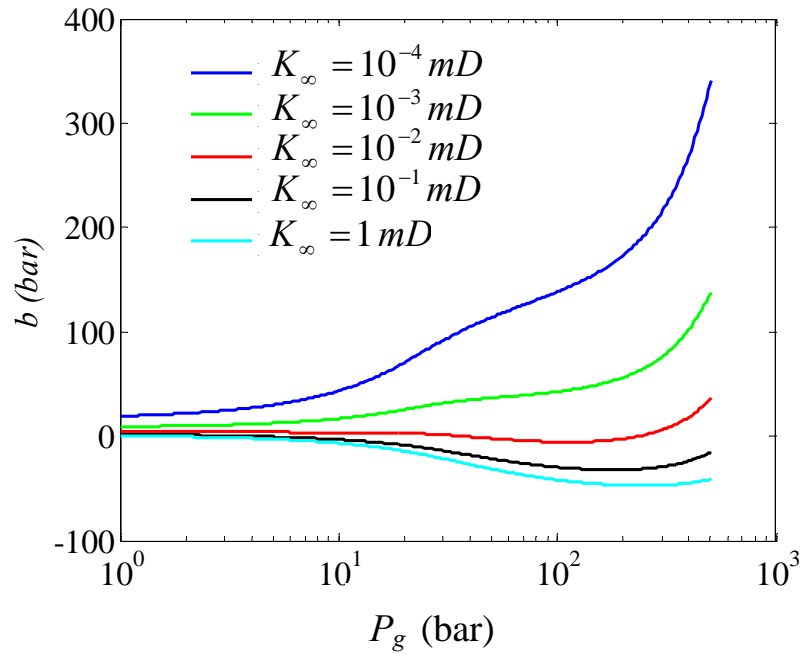

Figure 20. Klinkenberg parameter $b$ (from equation (101)) vs pressure for all permeabilities tested in the simulations. 
Figure 21 shows how the ratio of gas permeability to Klinkenberg permeability changes with the distance to the well for different values of permeabilities considered in our simulations. Notice that the magnitude of this ratio is directly the magnitude that the corresponding ratio of flow rate would have in all these scenarios.
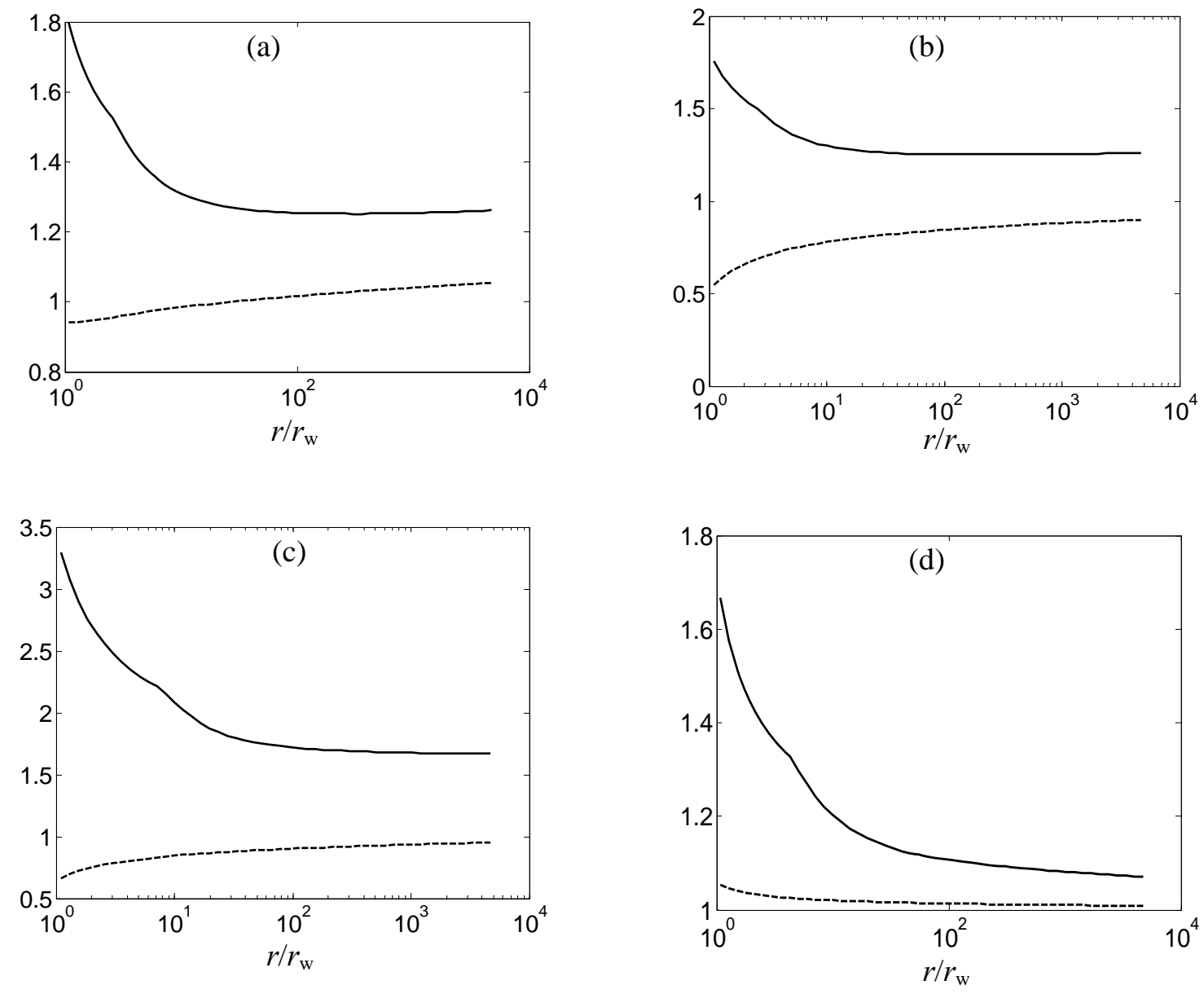

Figure 21. Ratio of Gas permeability to Klinkenberg permeability vs. distance to the well relative to the well radius. Continuous line for matrix $\left(K_{g}^{M} / K_{\infty}^{M}\right)$ and dashed line for fracture $\left(K_{g}^{F} / K_{\infty}^{F}\right)$.
(a) $K_{\infty}^{M}=10^{-3} \mathrm{mD}$ and $K_{\infty}^{F}=10^{-2} \mathrm{mD}$,
(b) $K_{\infty}^{M}=10^{-3} m D$ and $K_{\infty}^{F}=1 m D$,

$K_{\infty}^{M}=10^{-4} m D$ and $K_{\infty}^{F}=10^{-1} m D$, and (d) like (c) but with $b$ given by conventional approach (37). 
Sensitivity study to the different approaches to estimate Klinkenberg parameter $b$ is offer from Figure 22 to Figure 23. The effect on cumulated production in two scenarios: single-porosity singlepermeability and dual-porosity dual-permeability, under different relations between matrix and fracture permeabilities is illustrated. The permeability used to calculate the parameter $b$ is considered unaffected by the water saturation. Figure 4 in Ref. 89, show how this permeability is affected by the water saturation, however there is two typical forms of affecting the effective permeability due to the saturation. If the liquid water is occupying the smaller pores, or if it is forming films at pores walls. Both alternatives are valid when water is the wetting phase as it is expected in sands, however just when the water is forming films the reduction in permeability can be associated to a reduction in pore size. The correlation of Knudsen diffusion (and consequently of parameter $b$ ) with the Klinkenberg permeability is due to the correlation of this permeability with the pores size. Thus, only when reduction of this permeability is associated to a reduction in pore size, the Klinkenberg parameter $b$, would be affected. There is not evidence of how the fluids are distributed at pore scale, but it could be different depending of each particular case. In the following analysis we are not considering the effect of water saturation on the permeability and so, on the Klinkenberg parameter $b$. Nevertheless, cases where reduction on pore sizes can be demonstrated, the lector should consider the Klinkenberg permeability, of his case, affected in the right proportion.

Figure 22(a) and (b), which are obtained for $K_{\infty}^{F}=K_{\infty}^{M}=10^{-3} \mathrm{mD}$, are showing that a pressure dependent parameter $b$ increases the cumulated gas production in around $25 \%$ respect to the cumulated production predicted with a non-pressure dependent parameter $b$, as traditionally considered through equation (37). Thus an under-estimation of cumulated production, since early production times, of around $25 \%$ could be expected if the mentioned pressure dependence is ignored.

Figure 22(c) and (d), which resulted for $K_{\infty}^{F}=1000 K_{\infty}^{M}$ and $K_{\infty}^{M}=10^{-3} m D$, evidenced an overestimation of around $10 \%$ could be predicted if the pressure effect on the Klinkenberg parameter $b$ is overlooked. 
(a)

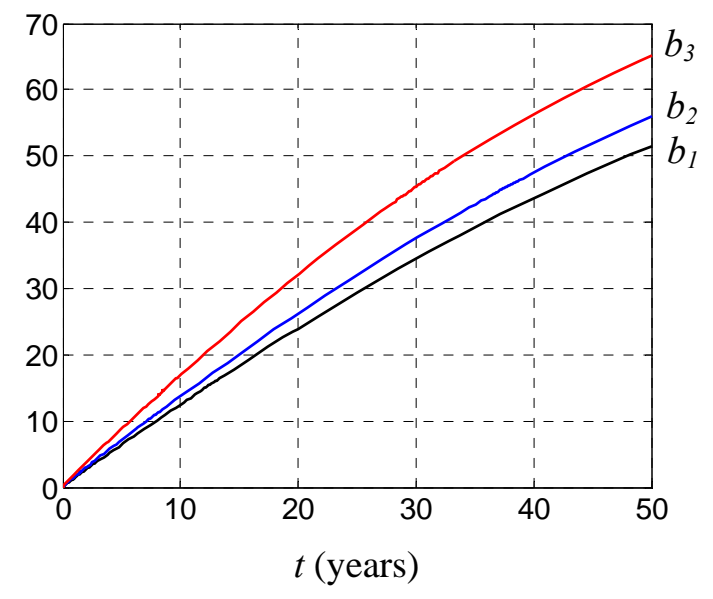

(c)

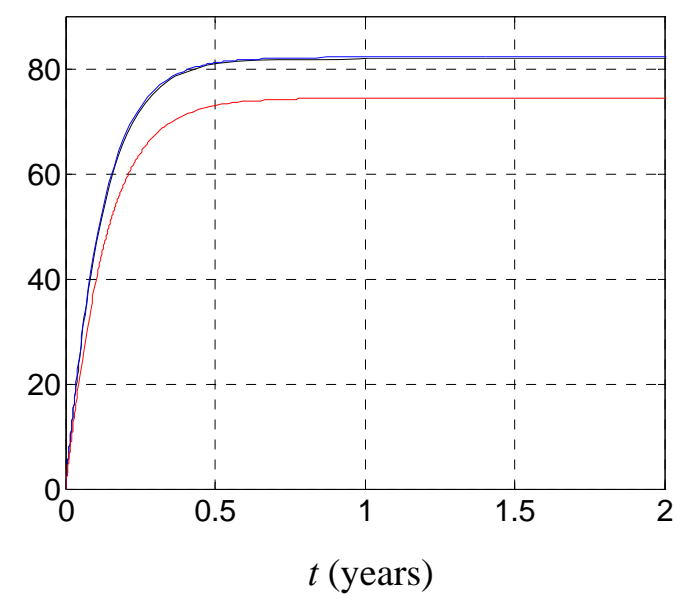

(b)

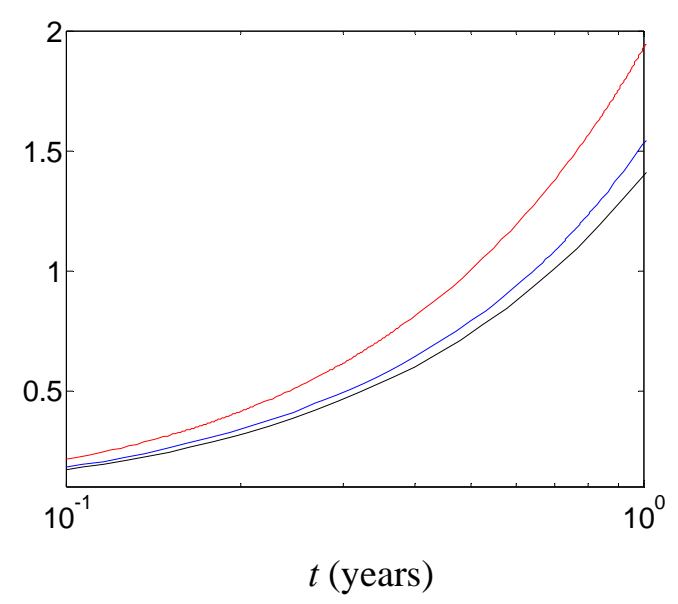

(d)

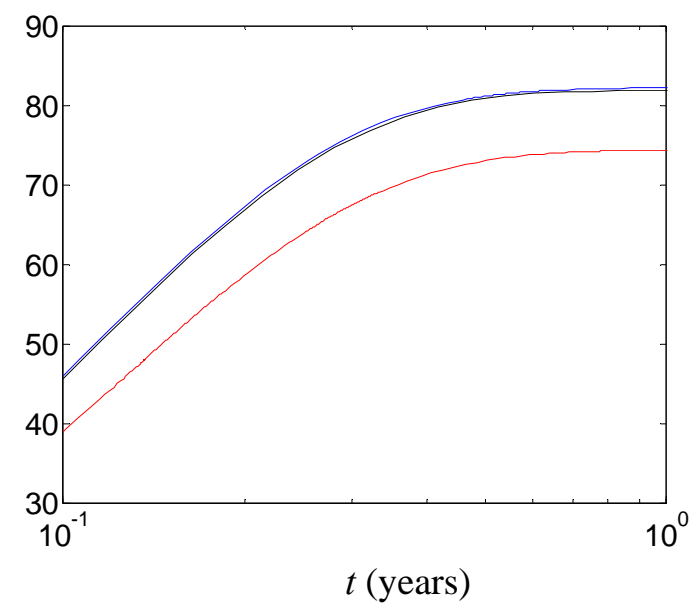

Figure 22. Cumulated gas production (MMSCF) vs time for three different $b: b_{1}=0, b_{2}$ given by equations (37), and $b_{3}$ dependent on pressure, by equation (101). (a) for base case, $K_{\infty}^{F}=K_{\infty}^{M}$, (b) plot (a) in a different scale, (c) $K_{\infty}^{F}=1000 K_{\infty}^{M}$ and (d) plot (a) in a different scale.

On the other hand, Figure 23(a) and (b), which are obtained for $K_{\infty}^{F}=K_{\infty}^{M}=10^{-4} \mathrm{mD}$, are presenting that an under-estimation of cumulated production, since early production times, of around 
$90 \%$ could be expected if the pressure dependence of $b$ is ignored and instead the traditional equation (37) is used to estimate this parameter.

(a)

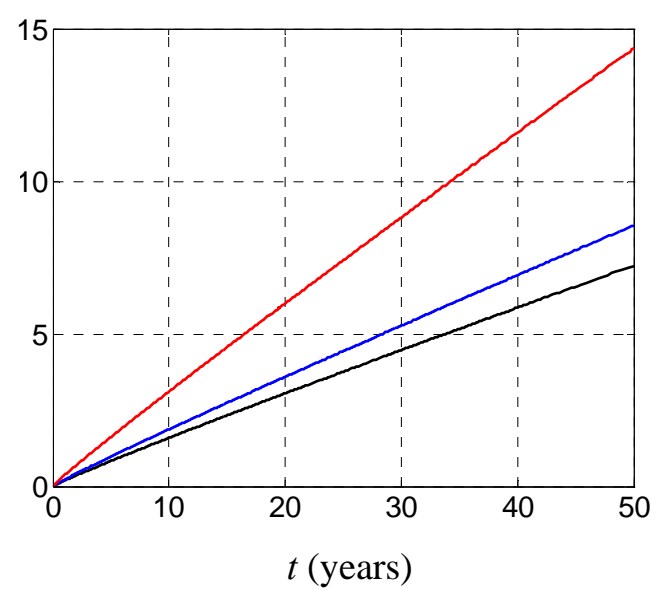

(a)

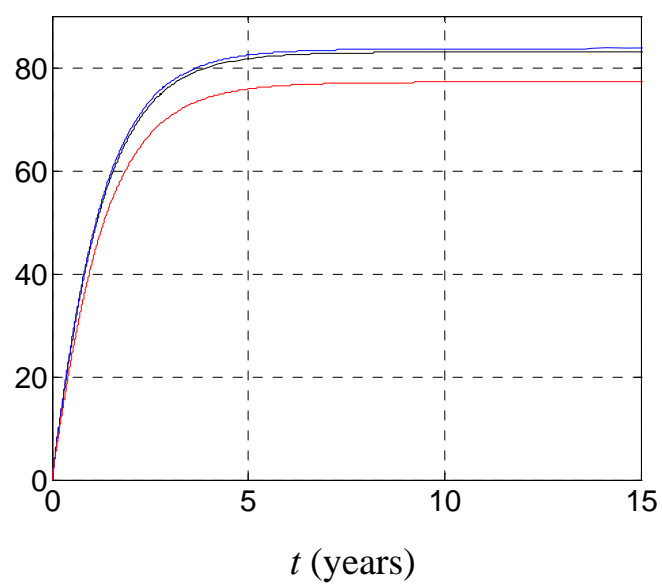

(b)

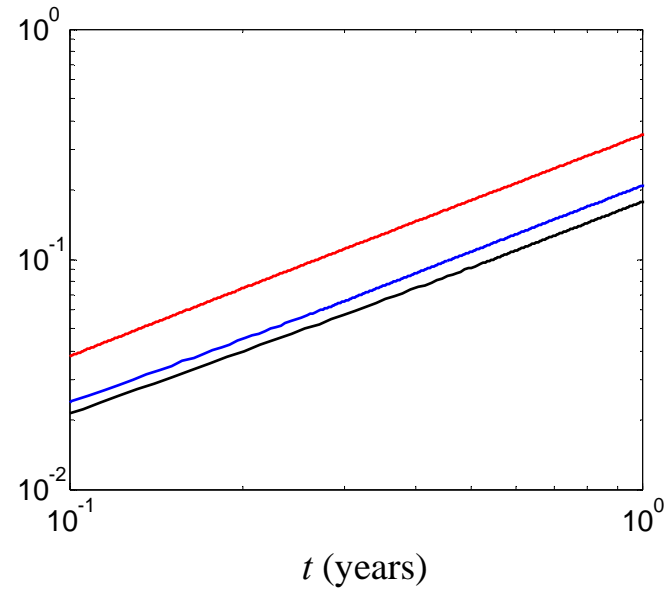

(b)

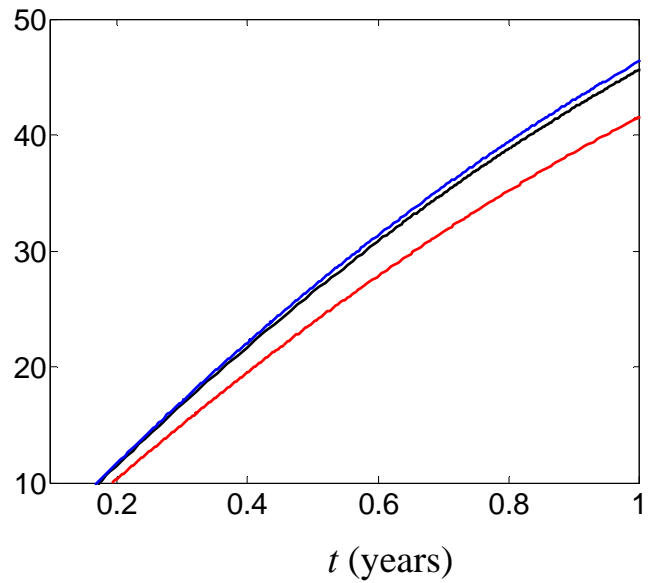

Figure 23. Cumulated gas production (MMSCF) vs time. $b_{1}=0, b_{2}$ given by equations (37), and $b_{3}$ $t$ (years)

dependent on pressure, by equation (101). (a) for $K_{\infty}^{M}=0.0001 m D, K_{\infty}^{F}=K_{\infty}^{M}$, (b) plot (a) in a different scale, (c) $K_{\infty}^{F}=1000 K_{\infty}^{M}$ and (d) plot (a) in a different scale.

Figure 23(c) and (d) were obtained for $K_{\infty}^{F}=1000 K_{\infty}^{M}$ with $K_{\infty}^{M}=10^{-4} \mathrm{mD}$. Curiously, the behavior observed was the same that in Figure 22(c) and (d). An overestimation of around $10 \%$ could be predicted if the pressure effect on the Klinkenberg parameter $b$ is overlooked. 
Sensitivities of the simulations to the flow mechanism were also performed in order to study how the relative contribution of diffusive and convective terms is under a dynamic simulation. The simulations showed that the diffusive contribution is a little less relevant than estimated from the magnitudes' order analysis. We observed that the cause of the over-estimation obtained in these preliminary analyses was the assumption expressed in the equations (63). In theses equations the water-methane moles number's ratio were considered varying only with pressure. The actual gradients of mole fractions in the simulations were different from the proposed through the approximations of equations (63).

Salt weight fraction $\left(\omega_{s}\right)$ was varied from 0 to 0.3 . Salt deposition was observed from values of around 0.10 and as consequence the permeabilities in both, matrix and fractures, were affected. A drastic effect of salt was observed for $\omega_{s}=0.3$. A reduction from more than 80 MMSCF to less than 4 MMSCF was obtained in cumulated gas production, due to salt depositions. Figure 24 shows these results.

(a)

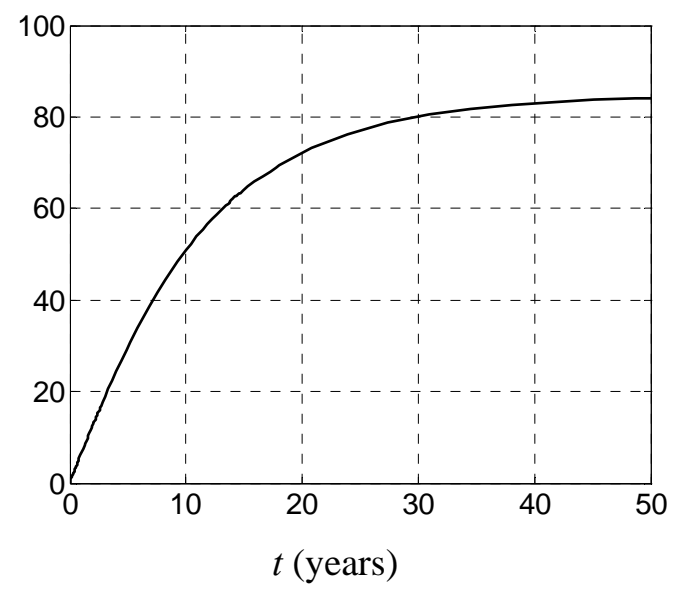

(b)

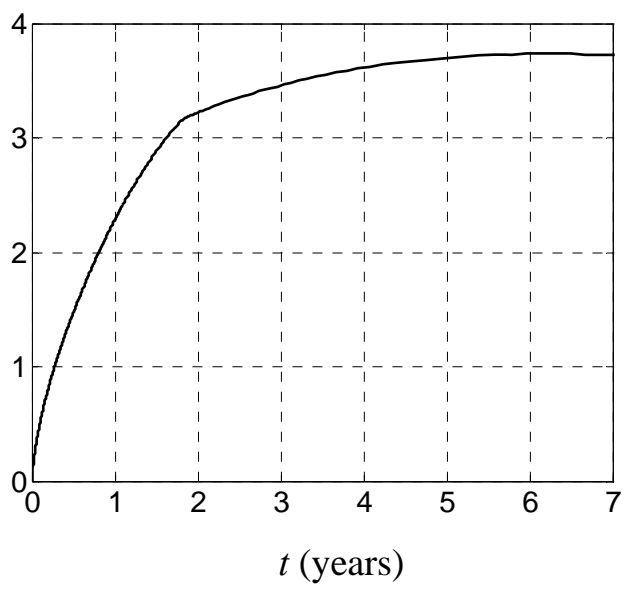

Figure 24. Cumulated gas production vs time. (a) $\omega_{s}=0$. (b) $\omega_{s}=0.3$

Sensitivities to the fracture longitude were also performed for $l$ varying between $10^{-4} \mathrm{~m}$ and $7 \mathrm{~m}$. Plots are not shown because the differences observed in the cumulated production were negligible. 


\section{CONCLUSION}

\subsection{Project AcComplishment}

A formulation based on a multi-mechanistic approach is proposed and evaluated for modeling of tight-sands gas reservoirs. The flow of methane in both phases, gas and liquid, was found to be impacted by the dispersive mechanism contribution in a non-negligible magnitude in general. This mechanism of flow was observed as dominating the dynamic for the lower range of pressure and Klinkenberg permeabilities evaluated. Moreover, when the contribution of the Knudsen Diffusion into the "convective" term was evaluated the actual contribution of the pure convective term turned on to be even smaller. The aforementioned results were based on magnitudes' orders analysis. The estimations were performed under unfavorable conditions for dispersion mechanism predominance $(\mathrm{Pe}<0.3)$. Thus, the contribution of dispersive mechanism of transport quantified here could be larger in various orders of magnitude. The vaporized water flow contribution is impacting the water transport, for typical temperatures of some tight sands reservoirs, in almost the whole range of pressures and permeabilites evaluated. Similar to methane gas flow, the water vapor flow was obtained from partially to totally controlled by the dispersive mechanism of transport.

The slippage effect and, more specifically, the way in which it is introduced in the macroscopic equations, was reviewed because of inconsistencies observed in the literature visited. We noticed that the so far ignored pressure dependence of the parameter $b$, could be of considerable relevance for the dynamic behavior of gases in the reservoirs. We derived an equation that allows considering the pressure effect on the parameter $b$. The equation predictions of $b$, as a dependent on pressure parameter were compared with experimental data reported for tight sands and other conventional porous media. Our model is capable of matching the experimental data, when a lineal velocity profile in the non-Poisseuille region is considered. Parabolic, constant and a combination of constant with linear were the others profiles tested.

The single previous work, that was found reported, which tried to explain the pressure dependence of the parameter $b$, concluded that the cause of this dependence was to consider a real gas. We already discussed the misinterpretations that originated this erroneous conclusion and now we can amend that conclusion. From our study, the pressure dependence of the Klinkenberg parameter $b$ is mostly due to the existence, at pore scale, of a region where neither Poisseuille nor Knudsen regime can be assumed. This region, which has been named in this report as a non-Poisseuille or transition region, encloses a flow affected by both, molecule-wall interactions and molecule-molecule interactions. 
Evaluations of the effect of the pressure dependence of $b$ in the gas flow rate showed that, for Knudsen numbers in the range $10^{-2}<\mathrm{Kn}<10^{2}$, the pressure dependence of $b$ can not be ignored, or, errors on flow rate predicted could be up to or larger than a $100 \%$. Out of this range of Knudsen numbers the assumption that $b$ does not depend on pressure, is a good approximation. It is worth mentioning that the effect of viscosity dependence on pressure was observed negligible for the pressures considered in the experiments used here.

We developed a 2-D numerical simulator, based on the proposed formalism, to evaluate on dynamic bases, the effect of diffusive mechanisms of flow (Knudsen and ordinary), water and methane phase transitions, and salt content variation on the predictions of fluid flow. Sensitivities studies showed that reservoirs simulators that ignore these aspects are unreliable in several tight sands reservoir conditions. The relevance of these aspects on the lack of prediction of conventional reservoir simulators is highly dependent on pressure, temperature, permeability and fractures density.

Simulation results indicate that if the pressure dependence of the Klinkenberg parameter $b$ is ignored, cumulated gas produced is under-predicted around $25 \%$ for matrix permeabilities of $0.001 \mathrm{mD}$. However, if this permeability just corresponds to the matrix permeability and the fracture permeability is considered 1000 times of it, instead of under-prediction we observe around $10 \%$ over-prediction, if the mentioned pressure dependence is ignored. Similar studies but for a permeability of $0.0001 \mathrm{mD}$, show that the under-prediction of cumulated gas production was around $90 \%$, while for fracture permeability of 10 times the matrix permeability the under-estimation is around $30 \%$. In this case if fracture permeability is 1000 times the matrix permeability an over estimation near of $10 \%$ is observed. Our estimations do not consider the effect of water saturation (around $40 \%$ in the case here studied) effect on the Klinkenberg permeability, because, in our opinion, further studies should be made on the fluids distribution at pore scale to determine if the reduction in permeability can be associated to a pore size reduction.

Performed sensitivities to the phase transitions of salts show that salts depositions, which reduce the permeability of matrix and fracture systems, were observed from a salt weight fraction near to 0.1 . A drastic reduction in cumulated gas production, from about 80 MMSCF to about 4 MMSCF was obtained for a salt weight fraction of 0.3 .

\subsection{RECOMMENDATIONS FOR FUTURE WORKS}

In terms of research and development works, further studies are needed, such as the following:

1. Develop experimental works focused on obtaining the pressure dependence of the Klinkenberg parameter $b$. The design of these experiments must guarantee that changes of 
gas permeability with pressure are only due to the molecules-wall interactions; in others words the net confining pressure must be constant during the experiments. Also, in order to obtain the Klinkenberg parameter $b$ from these data, it is imperative that the permeability to a non-polar liquid be measured on the same conditions. We demonstrated that $b$ is very sensitive to this permeability value (see Figure 15).

2. Obtain and justify the velocity profile in the transition region. Further evaluation of these profiles are needed, base on new experiments and advanced theoretical studies.

3. Explore causes of anomalous behaviors reported for relative permeabilities curves and their relation with water phase transition.

4. Model the changes of the electro-kinetic effect due to salt content variation due to the water phase transition in the reservoir.

5. Extend the simulator to 3-dimentional. Some sensitivity studies developed here could be different if the flow dynamic in the perpendicular direction to flow is involved and events, like water coning, are allowed.

In terms of reservoir engineering, we recommend to improve the expertise in those phenomena and events that become relevant and sometimes dominant of the flow dynamic for low-permeability reservoirs. Reservoir engineers unfamiliar with the way that these phenomena behave in each particular case could conduct to wrong production strategies.

Operators should note that low-permeabilities rocks reservoirs are unconventional, not simply because of the especial recovery process and technologies usually needed, but because of the way how the fluids dynamic occur. 


\section{NOMENCLATURE, ACRONYMS AND ABBREVIATIONS}

$b$._ Klinkenberg parameter.

$b_{0}$._ Klinkenberg parameter from conventional approach (equation (83) and (100)).

$b_{0 P}$._ $_{-}$Klinkenberg parameter from our pressure dependent approach (equation (101)).

$B_{i} \cdot$ Volumetric Factor of phase $i=g, l$; given by equations (17) and (18).

$c_{k} \cdot k_{\text {-component's molar concentration }}$

$C_{k \cdot-} k$-component's macroscopic molar concentration

$D_{k i} \cdot{ }_{-}$Molecular diffusion coefficient of component $k=m, w, s$ in phase $i=g, l$.

$D_{k i}^{*}$._ Dispersion coefficient of component $k=m, w, s$ in phase $i=g, l$.

$D_{k j}^{e f f}$._ Effective binary diffusivity coefficient $k, j=m, w, s$ (equation (76)).

$D^{K}$._ Knudsen diffusion coefficient of component $k$.

$F_{k-}=m_{k} / V_{T}$. Mass per unit of total volume of component $k=m, w, s \quad\left(\mathrm{~kg} / \mathrm{m}^{3}\right)$; defined by equations (4), (7) and (10).

$\vec{F}$._ Array of dimension $6 n_{r}$ with the $F_{k}$ for all the components in matrix and fractures in all numerical cells.

g._Gravity

$H_{m} \cdot-$ Henry constant for methane.

$K_{\infty} \cdot \_$Klinkenberg permeability.

$K_{i} \cdot$ - Absolute permeability of the porous system to phase $i=g, l$ for matrix or fractures.

$k r_{i} \cdot$ Relative permeability of phase $i=g, l, w, n w$.

$k r_{k i}$.- Relative permeability of phase $i=g, l, w, n w$; at irreducible saturation of the other phase.

Kn ._ Knudsen number.

$l$._ fracture spacing.

$L z$._ Formation thickness.

$m_{k} \cdot$ Mass of component $k=m, w, s$. 
$M$._ Generic mobility (convective or diffusive).

$M C_{k i} \cdot{ }_{-}$Convective mobility of component $k=m, w, s$ in phase $i=g, l$.

$M D_{k i} \cdot{ }_{-}$Diffusive mobility of component $k=m, w, s$ in phase $i=g, l$.

$M m_{k} \cdot-$ Molecular mass of component $k=m, w, s$.

$M m_{i} \cdot_{-}$Average molecular mass in phase $i=g, l$.

n._ Proportionality factor between velocity at wall and at boundary of the capillary (equation (91)).

$n_{k}$ : Molar density of component $i=m, w, s$.

$n_{k \cdot-}=\sum_{i} N_{k i} /\left(\phi V_{T}\right)$. Exponent in relative permeability correlation of phase $i=g, l$.

$n_{r} \cdot{ }_{-}$Number of intervals of the discretized region.

$\vec{n}$._ Array of dimension $6 n_{r}$ with the $n_{i}$ for all the components in matrix and fractures in all numerical cells

$N_{K}^{d}$._ Total molar diffusive flow of component $k$.

$N^{v}$._ Total molar viscous flow of component $k$.

$N_{k i} \cdot$ Number of moles of component $k=m, w, s$ in phase $i=g, l, s$.

$N_{T i}$-_ Total number of moles of all the components in phase $i=g, l, s$.

$N_{k T}$._ Total number of moles of component $k=m, w, s$ in all phases.

Pe._Peclet number.

$P^{*}$._ Non-dimensional pressure given by equation (103).

$P_{0}$._ Pressure value given by equation (102).

$P_{0}^{*}$._Non-dimensional reference pressure given by equation (103).

$P_{d} \cdot{ }_{-}$Displacement pressure in BCB correlation (48).

$P_{i} \cdot$ Pressure at phase $i=g, l, w, n w$.

$P_{i r}$._ $_{-}$Initial pressure in the reservoir, $i=g, l, w, n w$.

$P_{i w}$._ Pressure in the well. 
$P_{s w} \cdot$ - Saturation pressure of pure water.

$q_{i}$._ External mass input rate of component $k=m, w, s$ in a volume $V_{T}$.

$r_{0} \cdot$ See Figure 10 and equations (94).

$r_{b} \cdot$ - See Figure 10 and equations (95).

$r_{j} \cdot{ }_{-}$Radial coordinate, $j=0,1,2, \ldots, n_{r}, n_{r}+1$.

$R$._ Pore radius. See Figure 10

$R_{g} \cdot$ - Molecular (Universal) gas constant $(=8.314472 \mathrm{~J} / \mathrm{mol} \mathrm{K})$.

$R_{S g} \cdot-$ Vaporized liquid-gas ratio given by equation (19)

$R_{S l}$ •_ Dissolved gas-liquid ratio given by equation (20).

$S_{i} \cdot$ _ Saturation of phase $i=g, l, w, n w$.

$S_{i i} \cdot$ Irreducible saturation of phase $i=g, l, w, n w$.

$S_{r i} \cdot{ }_{-}$Reduced saturation of phase $i=g, l, w, n w$.

$t$._ Time.

$T$._ Temperature (K).

$T_{r} \cdot{ }_{-}$Reservoir Temperature (K).

$T_{k}^{M-F}$._ Mass transfer rate from matrix to fractures per unit of total volume given by equation (16).

$U_{k}$._ Mass input rate per unit of total volume of component $k=m, w, s$; as in equation (118).

$\vec{U}$._ Array of dimension $6 n_{r}$ with the $U_{i}$ for all the components in matrix and fractures in all numerical cells.

$\vec{u}$._ Mean pore velocity

$v_{0} \cdot$ - Velocity in the Knudsen layer. Related to $v_{b}$ through (87).

$v_{b} \cdot$ - Velocity in the boundary in between the transition and Poisseuille regions.

$v_{i} \cdot$ Velocity of phase $i=g, l$.

$V_{i}$._ Volume of phase $i=g, l, s$ at reservoir conditions. 
$V_{i}^{s c}$._ Volume of phase $i=g, l$ at standard conditions.

$V_{l v}^{s c} \cdot$ - Volume of liquid at standard conditions from vaporized water at reservoir conditions.

$V_{g d}^{s c} \cdot-$ Volume of gas at standard conditions from dissolved methane at reservoir conditions.

$\vec{V}_{N_{i}}^{k} \cdot-k$-component flow in the $i$-phase by the $N$-mechanism.

$V_{T} \cdot{ }_{-}$Total (rock + porous) volume.

$w$. $w \lambda$ is the thickness of the non-Poisseuille region in the capillary.

$x_{k i \cdot-}=N_{k i} / \sum_{k} N_{k i}$. Mole fraction in phase $i$ of component $k=m, w, s$.

$X$._ Driven parameter (pressure for convective flow or molar fraction for diffusive flow).

$z_{j} \cdot$ _L Logarithmic coordinate, $j=0,1,2, \ldots, n_{r}, n_{r}+1$, given by equation (119).

z ._ Flow direction in a capillary.

$Z$._ Real gas factor in the equation of state

\section{Greek symbols}

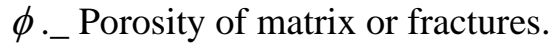

$\varphi_{x s}, \varphi_{y s} \cdot-$ Correction factors for salt content in equations (53) and (52), respectively.

$\xi$._ Sorting factor in BCB correlation (48).

$\lambda$._ Mean free path.

$\rho_{i} \cdot$ Density of phase $i=g, l$ at reservoir condition.

$\rho_{i}^{s c}$._. Density of phase $i=g, l$ at standard conditions.

$\rho_{w}^{s c} \cdot \_$Density of pure water (liquid) at standard conditions.

$\omega$._ Acentric factor.

$\omega_{s} \cdot-$ Salt weight fraction in depressurized liquid given by equation (21) .

\section{Subscript:}

c._ critical point

C._Convective

D._ Dispersive 
$f$._freezing point

$g$._ Gas Phase

$K n$._ Knudsen

$l$._ Liquid phase

$P C$._ Pure Convective (without Knudsen’s contribution)

$s$._ Solid phase or salt

$w$._ Wetting phase or water

$m$. Methane

$n w$._ Non-wetting phase

$T$._ Total

\section{Super-script:}

$M$._ Matrix

$F$._ Fracture

Well._Well of production

\section{Acronyms}

ACR ._Applied Research Center

2-D._ Two-dimensional

VOC._ Volatile Organic Compound

NAPL._ Non-Aqueous Phase Liquid

BOAST._ Black Oil Applied Simulation Tool

PVT._Pressure-Temperature-Volume

EOS._Equation of State

ADM._Advection Diffusion Model

DGM._Dusty Gas Model 


\section{ACKNOWLEDGMENT}

The assistance of Marcelo Lago of University of Miami in the development of the simulator, and his support in technical discussions is highly appreciated. 


\section{REFERENCES}

${ }^{1}$ Energy Information Administration. (Dec-2005). Annual Energy Outlook 2006, http://www.eia.doe.gov/oiaf/aeo/pdf/aeotab_13.pdf.

${ }^{2}$ Munka, M., Papay, J., (2001) “4 D Numerical modeling of petroleum reservoir recovery”, Akademiai Kiado, Budapest.

${ }^{3}$ Sung W. and Ertekin T., (1986) “The Development, Testing, and Application of a Comprehensive Coal Seam Degasification Model”, Unconventional Gas Technology Symposium of the SPE, Louisville, KY, May 1821,. (SPE-15247)

${ }^{4}$ Ertekin, T., King, G. R. and Schwerer, F. C., (1986) "Dynamic gas slippage: A unique dual-mechanism approach to the flow of gas in tight formations”, SPE-Formation evaluation, Feb, 43-52.

${ }^{5}$ Chawathe A., Ertekin T., Grader A. (1996) "Understanding Multi-mechanistic Gas-Water flow in Fractured Reservoirs: Mapping of the Multi-Mechanistic Flow Domain”. SPE (36738) Annual Technical Conference and Exhibition, Denver, Colorado, USA, 6-9 Oct.

${ }^{6}$ Jalali, J. and Mohaghegh, S. D., (September 2004) “A Coalbed Methane Reservoir Simulator Designed and Developed for the Independent Producers”. SPE (91414) Eastern Regional Meeting, Charleston, West Virginia, U.S.A., 15-17.

${ }^{7}$ National Petroleum Council, (1980) “Unconventional Gas Sources“, Vol 5 (Tight Gas Reservoirs) part 1.

${ }^{8}$ Krishna R. and Wesselingh J. A., (1997) “The Maxwell-Stefan Approach to Mass Transfer,” Chem. Eng. Sci., 52,861

9 Rathfelder, K., Lang, J. and Abriola, L.M., (1995) "Soil Vapor Extraction and Bioventing: Application, Limitations and Future Research Directions, U.S. National Report to IUGG, 1991-1994”, Rev. Geophys., 33 Suppl., 1067.

${ }^{10}$ Hongkyu Y., Valocchi A. J. and Werth C. J., (2003) “Modeling the Influence of Water Content on Soil Vapor Extraction,” Vadose Zone Journal, 2, 368.

${ }^{11}$ Ravikrishna, R., Valsaraj K.T., Thibodeaux L.J., Reible D.D., (2001) “Air Emission Flux from Contaminated Dredged Materials Stored in a Pilot-Scale Confined Disposal Facility”, J. of the Air \& Waste Management Association, 51, 174.

12 Klinkenberg, L. J., (1941) “The Permeability of Porous Media to Liquids and Gases”, Drilling and Production Practice, Am. Petrol. Inst., 200.

${ }^{13}$ Li, S., Dong, M., Dai, L., Li, Z. and Pan X., (2004) "Determination of Gas Permeability of Tight Reservoir Cores without Using Klinkenberg Correlation”, SPE- 88472. 
${ }^{14}$ Chowdiah, P., (1986) “Effects of Pore Water Distribution and Stress on the Laboratory Measurement of Tight Sandstone Properties,” SPE-15210.

15 Soeder, D.J. Chowdiah, P., (1990) “Pore Geometry in High- and Low-Permeability Sandstones, Travis Peak Formation, East Texas,” SPE- 17729.

16 Jones, F.O. and Owens, W.W., (1981) “A Laboratory Study of Low-Permeability Gas Sands,” SPE-7551.

${ }^{17}$ Randolph, P.L., Soeder, D.J., Chowdiah, P., (1984) “Porosity and Permeability of Tight Sands,” SPE- 12836.

18 Brewer, K.R., Morrow, N.R., (1985) "Fluid Flow in Cracks as Related to Low-Permeability Gas Sands,” SPE-11623.

${ }^{19}$ Wang, C-H., (1992) “Non-Darcy flow analysis through tight sand formations“ Thesis (Ph.D.), Illinois Inst. of Tech., Chicago, IL.

${ }^{20}$ Sampath, K. and Willian K.C., (1982) "Factors Affecting Gas Slippage in Tight Sandstones of Cretaceous Age in the Uinta Basin,” SPE-9872.

21 Morrow, N.R., Buckley, J.S., Cather, M., Brower, K.R., Bretz, R., Gonzales, B., Graham, M., Kinner, R., Ma, S., Voigt, B. and Zhang, X., (1989) "Rock Matrix and Fracture Analysis of Flow in Western Tight Gas Sands,” Phase 4, DOE/MC/21179-2710.

22 Newsham, K.E, Rushing, J.A., (2002) "Laboratory and Field Observation of an Apparent Sub Capillary Equilibrium Water Saturation Distribution in a Tight Gas Reservoir,” SPE-75710.

23 Arastoopour H And Hariri H., (1987) “Analysis Of Two-Phase Flow in a Tight Sand Gas Reservoir, Using Boast (Black Oil Applied Simulation Tool),” SPE-15837.

${ }^{24}$ Holditch, S.A., Lin, Z-S. and Spivey, J.P., (1991) “Estimating the Recovery From an Average Well in a Tight Gas Formation,” SPE-21500.

25 De Swaan, A,, Berumen, S, and Rodriguez F., (2002) "Reservoir and Surface-Network Simulation Of Tight Gas Reservoirs In The North Of Mexico,” SPE-74420.

26 Newberg, M. A. and Arastoopour, H., (1986) “Analysis of the Flow of Gas Through Low-Permeability Porous Media,” SPE-14705.

${ }^{27}$ Guo, X., Du, Z. and Li, S.., (2003) “Computer Modeling and Simulation of a Coal Bed Methane Reservoir,” SPE-84815.

28 Bezilla, M., Ertekin, T., Adewumi, M.A. and Frohne, K.H., (1989) "Multi-mechanistic Approach to the Reservoir Analysis of Tight Blanket Sands, SPE- 18983.

${ }^{29}$ Chen, S-T., (1989) "Numerical Analysis of Gas-Liquid Flow through Porous Medium: in Application with Gas Reservoir”, Ph.D. Thesis, Illinois Inst. of Tech., Chicago. 
30 Arastoopour, H. and Chen, S-T., (1991) "Sensitivity Analysis of Key Reservoir Parameters in Gas Reservoirs,” SPE 21515.

31 Carlson, E.S. and Latham, G.V., (1993) “Discrete network modeling of tight gas fractured reservoirs;” SPE26122.

32 Ubani, E., (1985) “The Well Performance of Naturally Fractured Lenticular Sand Reservoirs,” PhD Thesis, University of Oklahoma.

33 Evans, R.D. and Lekia, S.D.L., (1990) “A Eeservoir Simulation Study of Naturally Fractured Lenticular Tight Gas Sand Reservoirs, J. Energy Res. Tech. ; 231.

34 Oda, M., (1985) “Permeability Tensor for Discontinuous Rock Masses,” Geotechnique, 35, 483.

35 Harstad, H., Teufel, L. W. and Lorenz, J. C., (1995) “Characterization And Simulation Of Naturally Fractured Tight Gas Sandstone Reservoirs, SPE- 30573.

36 Lough, M. F., Lee, S. H. and Kamath, J., (1998) “An Efficient Boundary Integral Formulation for Flow Through Fractured Porous Media“ J. Comp Phys. 143, 462.

${ }^{37}$ Davies, J. P. and Davies, D. K., (1999) “Stress-Dependent Permeability: Characterization and Modelling,” SPE-56813.

38 Evans, R. D., (1981) “Characterization of fluid flow in naturally fractured reservoirs”, DE-RP19-80BC10255, Final Report.

${ }^{39}$ Settari, A., Sullivan, R. B. and Bachman, R. C, (2002) "The Modeling of the Effect of Water Blockage and Geomechanics in Waterfracs, SPE- 77600.

${ }^{40}$ Danesh A., (2003) "PVT and Phase Behaviour of Petroleum Reservoir Fluids: Developments in Petroleum Science 47, Elsevier, Third Edition.

${ }^{41}$ Zuo, Y.-X., T.-M. Guo, (1991) "Extension of the Patel-Teja Equation of State to the Prediction of the Solubility of Natural Gas in Formation Water”, Chemical Engineering Science 44 (12), 3251.

${ }^{42}$ Zuo, Y.-X., E. H. Stenby, T.-M. Guo, (1996) "Simulation of the high-pressure phase equilibria of hydrocarbon-water/brine systems” Journal of Petroleum Science and Engineering 15, 201-220.

${ }^{43}$ Masoudi, R., M. Arjmandi, B. Tohidi, (2003) "Extension of Valderrama-Patel-Teja equation of state to modeling single and mixed electrolyte solutions”, Chemical Engineering Science 58, 1743.

${ }^{44}$ Masoudi, R., B. Tohidi, A. Danesh, A. C. Todd, (2004) “A new approach in modelling phase equilibria and gas solubility in electrolyte solutions and its applications to gas hydrates”, Fluid Phase Equilibria 215, 163.

45 Battistelli, A.; C. Calore, K. Pruess, (1997) “The Simulator Tough2/Ewasg for Modelling Geothermal Reservoirs with Brines and Non-Condensible Gas”, Geothermics 26 (4), 437. 
46 Thorstenson, D. C., D. W. Pollock, (1989) “Gas Transport in Unsaturated Zones: Multicomponent Systems and the Adequacy of Fick’s laws”, Water Resources research 25, 477.

47 Brooks, R. H., A. T. Corey, (1964) “Hydraulic properties of porous media” Hydrology Papers, Colorado State University, 24 p.

48 Corey, A. T., (1994) “Mechanics of Immiscible Fluid in Porous Media”, WRP; 3 rd ed.

${ }^{49}$ Fried, J. and Combarnous, M. A., (1971) “Dispersion in Porous Media,” Advances in Hydroscience, 7, 169.

50 Bear, J., (1988) “Dynamics of fluids in porous media” Dover Publication Inc., New York, 608.

51 Sahimi, M., (1993) "Flow phenomena in rocks: from continuous models to fractals, percolation, cellular automata, and simulated annealing”, Review of modern Physics, 65 (4) 1393.

${ }^{52}$ Lee H. and Thodos G., (1983) “Generalized Treatment of Self-Diffusivity for the Gaseous and Liquid States of Fluids”. Ind. Eng. Chem. Fundam., 22, 17-26.

${ }^{53}$ Hayduk W. and Laudie H. (1974) AlChE Journal, 20, May, 611.

54 Starling, K. E., (1973) “Fluid Thermodynamic Properties for Light Petroleum Systems” Gulf publishing, Houston.

55 Batzle, M., Z. Wang, (1992) Seismic properties of pore fluids: Geophysics 57 (11), 1396.

${ }^{56}$ Adams, J. J., S. Bachu, (2002) "Equations of state for basin geofluids: algorithm review and intercomparison for brines” Geofluids 2, 257.

57 Daubert, T. E., R. P. Danner, (1985) DIPPR Data Compilation Tables of Properties of Pure Compounds: AIChE, New York.

${ }^{58}$ McCain Jr., W. D., (1990) “The properties of Petroleum Fluids”, 2nd edition, Pennwell Books, Tulsa.

59 Voutsas, E. C., G. C. Boulougouris, I. G. Economou, D. P. Tassios, (2000) “Water/Hydrocarbon Phase Equilibria Using the Thermodynamic Perturbation Theory” Industrial and Engineering Chemistry Research 39, 797.

${ }^{60}$ Morin, E., F. Montel, (1995) Accurate predictions for the production of vaporized water: Paper presented at 1995 SPE Annual Technical Conference and Exhibition, 22-25 October, Dallas, Texas. SPE paper no. 30719.

61 Harvey, A. H., (1996) “Semiempirical correlation for Henry's constants over large temperature ranges” AIChE Journal 42 (5), 1491.

${ }^{62}$ Lee, A. L., M. H. Gonzalez, B. E. Eakin, (1966) “The viscosity of natural gases”, Journal of Petroleum and Technology, 997-1000. SPE-1340.

63 Palliser C., R. Mckibbin (1998) “A Model for Deep Geothermal Brines, I: T-p-X State-Space Description” Transport in Porous Media 33, 65. 
${ }^{64}$ Kestin, J., H. E. Khalifa, R. J. Correia, (1981) "Tables of dynamic and kinematic viscosity of aqueous NaCl solutions in the temperature range 20-150 ${ }^{\circ} \mathrm{C}$ and the pressure range 0.1-35 MPa” Journal of Physical Chemistry Reference Data 10, 71-87.

${ }^{65}$ Roy, S. and R. Raju, (2003) “Modeling Gas through Microchannels and Nanopores”, J. App. Phys., 93, pp. 4870-4879.

${ }^{66}$ Karniadakis, G., Beskok, A. and Aluru, N., (2005) "Microflows and Nanoflows Fundamentals and Simulation," Springer.

${ }^{67}$ Skjetne, E. and J. L. Auriault; (1999) "Homogenization of Wall-Slip Gas Flow Through Porous Media;," Transport in Porous Media 36: 293-306.

${ }^{68}$ Chastanet, J., P. Royer and J.-L. Auriault, (2004) “Does Klinkenberg’s Law Survive Upscaling?” Transport in Porous Media, 56, 171-198.

${ }^{69}$ Piet J. A. M. Kerkhof and Marcel A. M. Geboers, (2005) "Toward a Unified Theory of Isotropic Molecular Transport Phenomena,” AIChE Journal, 51(1) 79-121.

${ }^{70}$ Mason, E. A. and Malinauskas, A. P. (1983) “Gas transport in porous media; the dusty-gas model” Chem. Eng. Monogr., Vol.17, Elsevier, New York.

${ }^{71}$ Sleep, B. E. (1998) "Modeling transient organic vapor transport in porous media with the Dusty Gas Model," Advances in Water Resources, 22, 247-256.

${ }^{72}$ Reinecke, S. A. and B. E. Sleep, (2002) "Knudsen Diffusion, gas permeability, and water content in an unconsolidated porous medium,” Water Resources Res., 38, 1280.

${ }^{73}$ Webb, S. and K. Pruess, (2003) “The Use of Fick's Law for Modeling Trace Gas Diffusion in Porous Media,” Transport in Porous Media, 51, 327.

${ }^{74}$ Abu-El-Sha'r, W. and L. M. Abriola, (1997) “Experimental assessment of gas transport mechanisms in natural porous media: Parameter evaluation,” Water Resour. Res. 33, 505-516.

${ }^{75}$ Chiu-Shia Fen , Linda. M. Abriola, (2004) “A comparison of mathematical model formulations for organic vapor transport in porous media” Advances in Water Resources 27 1005-1016.

${ }^{76}$ Olderburg, C. M., S. W. Webb, K. Pruess and G. J. Moridis, (2004) "Mixing of Stably Stratified Gases in Subsurface Reservoirs: A Comparison of Diffusion Models” Transport in Porous Media 54: 323-334.

${ }^{77}$ Webb, S. W.; (1998) "Gas diffusion in porous media- evaluation of an advective-dispersive formulation and the Dusty gas Model for binary mixtures,” J. Porous media, 1, 187-199.

${ }^{78}$ Wu, Y.-S, K. Pruess and P. Persoff; (1998) “Gas flow in porous media with Klinkenberg effects,” Transport Porous Media 32, 117-137. 
79 Mason, E. A. Malinauskas, A. P. and Evans R. B., (1967) “Flow and Diffusion of Gases in Porous Media,” The Journal of Chemical Physics, 46 (8), 3199.

${ }^{80}$ Gombosi , T. I., (1994) “Gaskinetic Theory,” Cambridge University Press.

81 Do, D. D., (1998) “Adsorption Analysis: Equilibria and Kinetics,” Series Chem. Eng. 2. Imperial College press.

${ }^{82}$ Burganos, V. N., (1998) “Gas difussion in random binary media,” J. of Chemical Physics, $109,6772$.

83 L. C. Woods, An introduction of the Kinetic Theory of gases and magnetoplasmas, Oxford University Press Inc, New York, 1993.

${ }^{84}$ Mattax C. C., R. L. Dalton, 1990. Reservoir Simulation: SPE Monograph Series 13, SPE, Richardson, TX.

${ }^{85}$ N. L. Carr, R. Kobayaski and D. B. Burrows, Viscosity of hydrocarbon gases under pressure, Trans. AIME, pp. 264-72, 1954.

${ }^{86}$ Hall, K. R. and Yarborough, L., A new equation of state for Z-factor calculations, Oil and Gas J., Jun 18, $82-$ 92,1973

${ }^{87}$ R. H. Perry and D. W. Green, Perry’s Chemical Engineering Handbook, Seventh edition, Mc Graw Hill, 1997.

88 J.A. Rushing, K.E. Newsham and K.C. Van Fraassen; Measurement of the two-phase gas slippage phenomenon and its effect on gas relative permeability in tight gas sands; SPE-84297; 2003.

89 Branagan, P., Cotner, G. and Lee, S.J., (1984) "Interference testing of the naturally fracture cozzette sandstone: A case study at the DOE MWX site”, SPE-12869 